\title{
Extracellular vesicles as a drug delivery system: A systematic review of preclinical studies
}

\author{
Pol Escudé Martinez de Castilla ${ }^{\mathrm{a}, 1}$, Lingjun Tong ${ }^{\mathrm{b}, \mathrm{c}, 1}$, Chenyuan Huang ${ }^{\mathrm{b}, \mathrm{c}, 1}$, Alexandros Marios Sofias ${ }^{\mathrm{d}}$, \\ Giorgia Pastorin $^{\mathrm{e}}$, Xiaoyuan Chen ${ }^{\mathrm{b}, \mathrm{f}, \mathrm{g}, \mathrm{h}, \mathrm{i}}$, Gert Storm ${ }^{\mathrm{b}, \mathrm{i}, \mathrm{j}, \mathrm{k}}$, Raymond M. Schiffelers ${ }^{\mathrm{a}, *}$, \\ Jiong-Wei Wang ${ }^{\mathrm{b}, \mathrm{c}, \mathrm{i}, \mathrm{l}, *}$
}

a CDL Research, University Medical Center Utrecht, 3584 CX Utrecht, the Netherlands

${ }^{\mathrm{b}}$ Department of Surgery, Yong Loo Lin School of Medicine, National University of Singapore, 119228 Singapore, Singapore

${ }^{\mathrm{c}}$ Cardiovascular Research Institute (CVRI), National University Heart Centre Singapore (NUHCS), 117599 Singapore, Singapore

${ }^{\mathrm{d}}$ Institute for Experimental Molecular Imaging, Faculty of Medicine, RWTH Aachen University, 52074 Aachen, Germany

e Department of Pharmacy, Faculty of Science, National University of Singapore, 117543 Singapore, Singapore

${ }^{\mathrm{f}}$ Department of Diagnostic Radiology, Yong Loo Lin School of Medicine, National University of Singapore, 119074 Singapore, Singapore

${ }^{\mathrm{g}}$ Departments of Chemical and Biomolecular Engineering, and Biomedical Engineering, Faculty of Engineering, National University of Singapore, 117575 Singapore, Singapore

${ }^{\mathrm{h}}$ Clinical Imaging Research Centre, Centre for Translational Medicine, Yong Loo Lin School of Medicine, National University of Singapore, 117599 Singapore, Singapore

${ }^{\mathrm{i}}$ Nanomedicine Translational Research Programme, Centre for NanoMedicine, Yong Loo Lin School of Medicine, National University of Singapore, 117609 Singapore, Singapore

j Department of Pharmaceutics, Faculty of Science, Utrecht University, 3584 CG Utrecht, the Netherlands

${ }^{\mathrm{k}}$ Department of Biomaterials, Science and Technology, Faculty of Science and Technology, University of Twente, 7522 NB Enschede, the Netherlands

${ }^{1}$ Department of Physiology, Yong Loo Lin School of Medicine, National University of Singapore, Singapore 117593, Singapore

\section{A R T I C L E I N F O}

\section{Article history:}

Received 18 February 2021

Revised 10 May 2021

Accepted 15 May 2021

Available online 18 May 2021

\section{Keywords:}

Extracellular vesicles

Liposomes

Systematic review

Drug delivery

Preclinical animal models

Clinical trial

\begin{abstract}
A B S T R A C T
During the past decades, extracellular vesicles (EVs) have emerged as an attractive drug delivery system. Here, we assess their pre-clinical applications, in the form of a systematic review. For each study published in the past decade, disease models, animal species, EV donor cell types, active pharmaceutical ingredients (APIs), EV surface modifications, API loading methods, EV size and charge, estimation of EV purity, presence of biodistribution studies and administration routes were quantitatively analyzed in a defined and reproducible way. We have interpreted the trends we observe over the past decade, to define the niches where to apply EVs for drug delivery in the future and to provide a basis for regulatory guidelines.
\end{abstract}

(C) 2021 Elsevier B.V. All rights reserved.

\section{Contents}

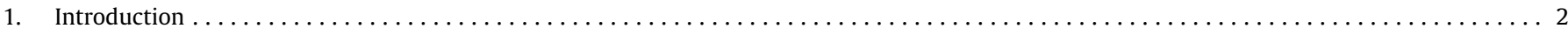

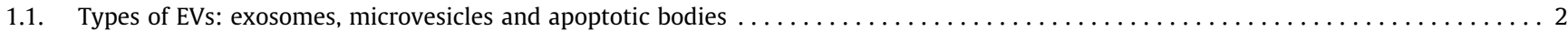

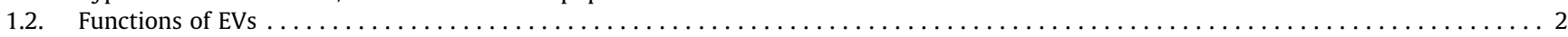

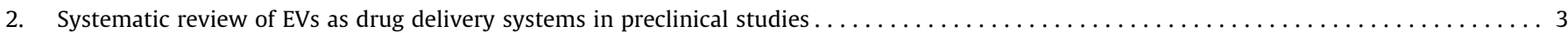

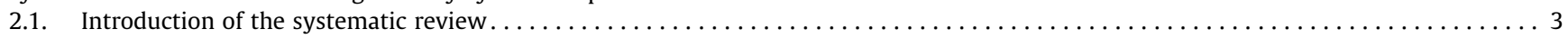

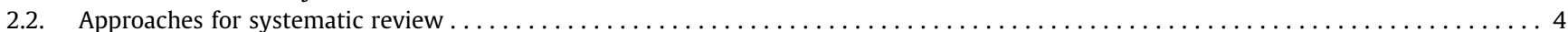

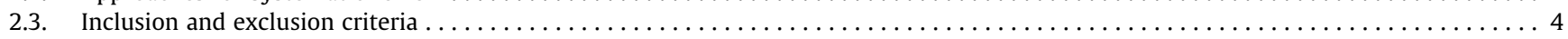

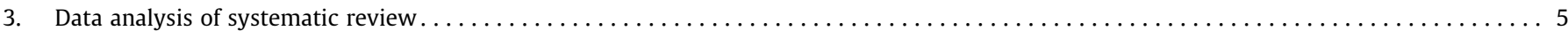

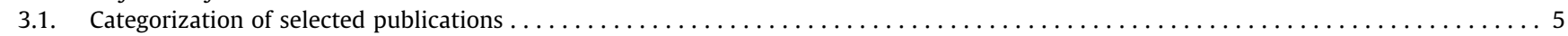

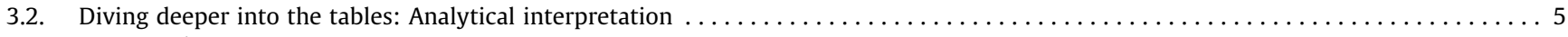

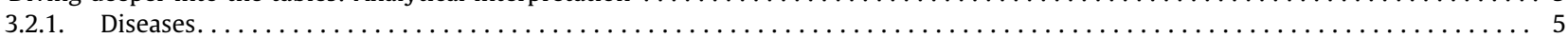

\footnotetext{
* Corresponding authors at: Department of Surgery, Yong Loo Lin School of Medicine, National University of Singapore, 119228 Singapore, Singapore (J.W. Wang).

E-mail addresses: R.Schiffelers@umcutrecht.nl (R.M. Schiffelers), surwang@nus.edu.sg (J.-W. Wang).

1 These authors contributed equally.
} 


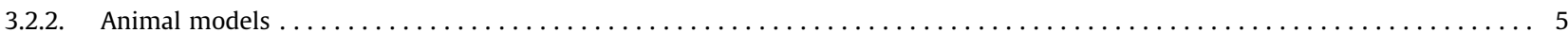

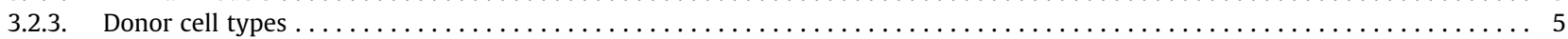

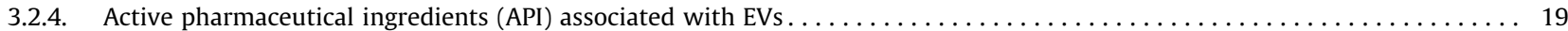

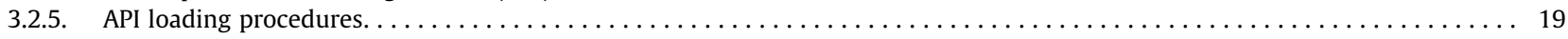

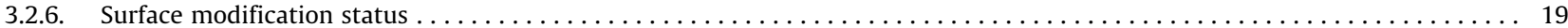

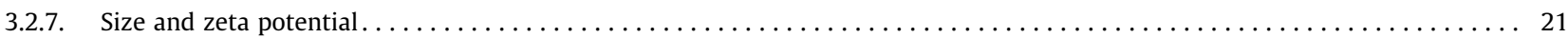

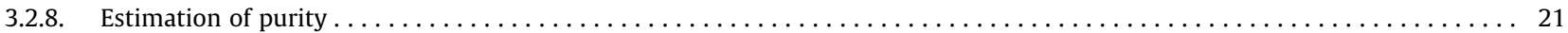

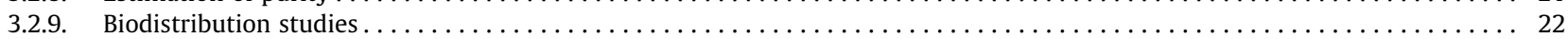

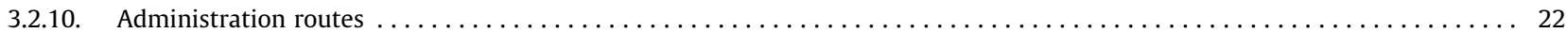

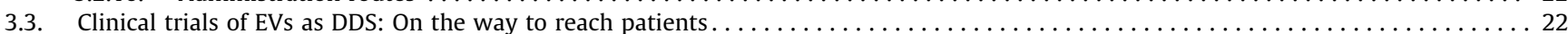

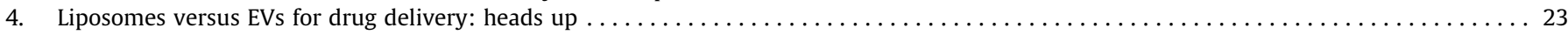

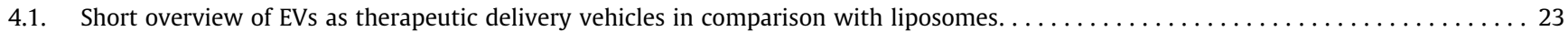

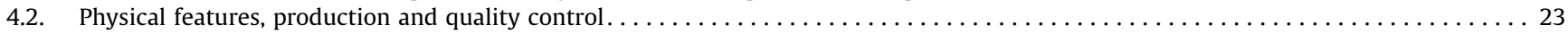

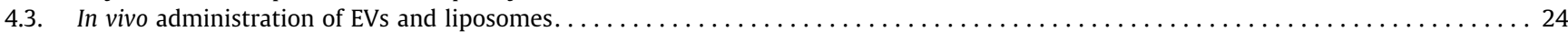

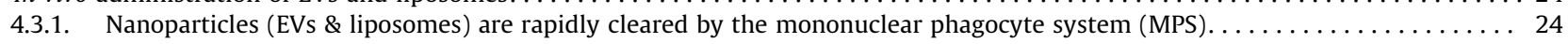

4.3.2. Accelerated blood clearance $(\mathrm{ABC})$ phenomenon upon multiple injections of nanoparticles $\ldots \ldots \ldots \ldots \ldots \ldots \ldots \ldots \ldots \ldots$

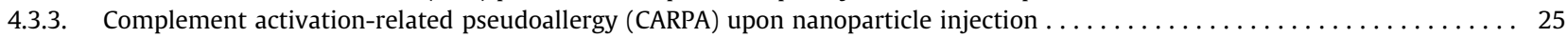

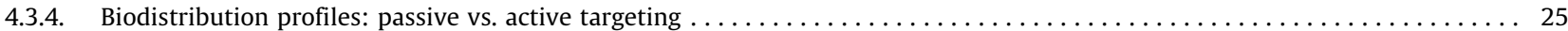

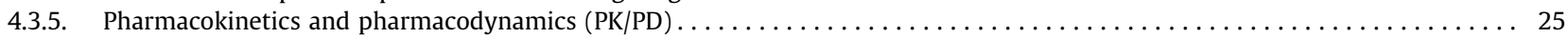

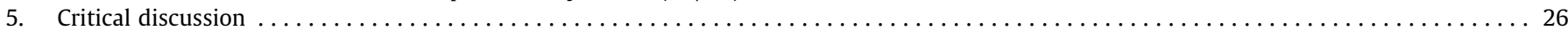

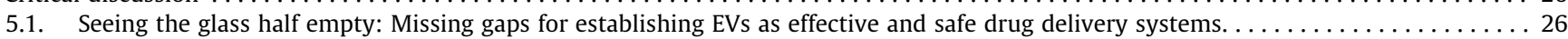

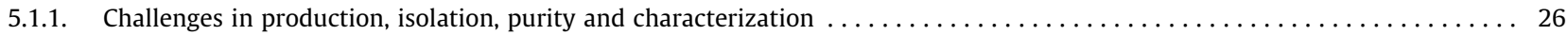

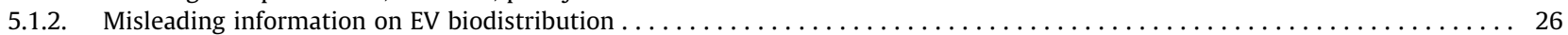

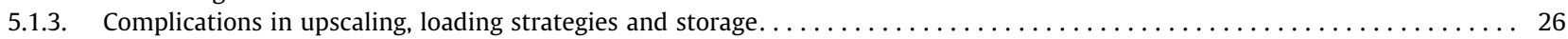

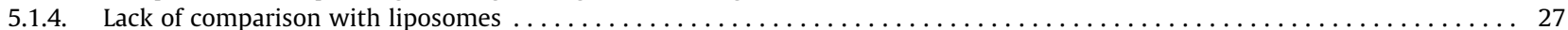

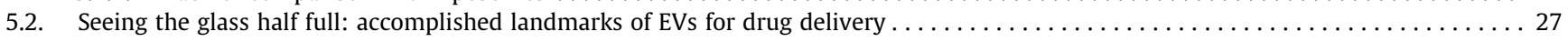

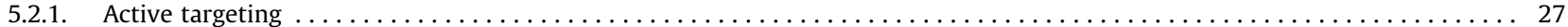

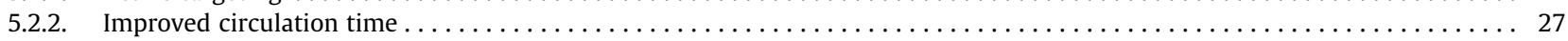

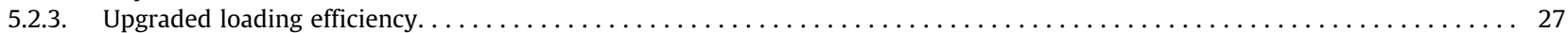

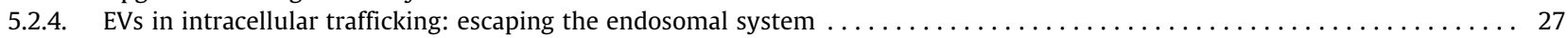

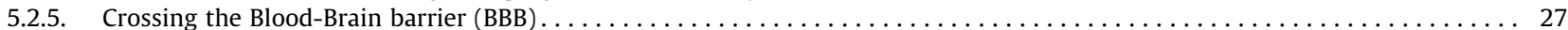

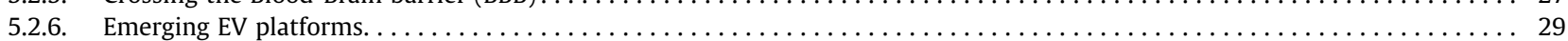

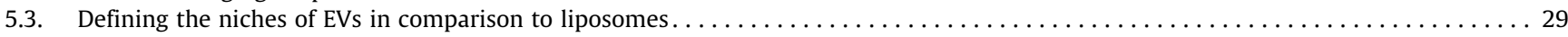

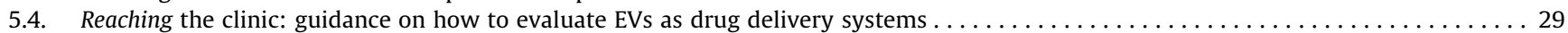

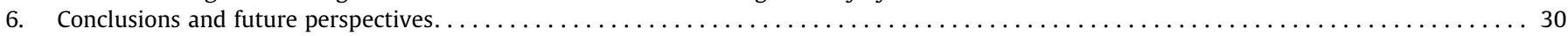

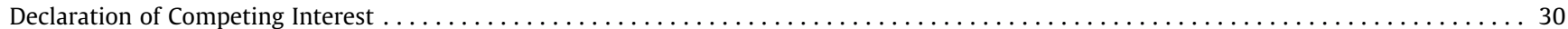

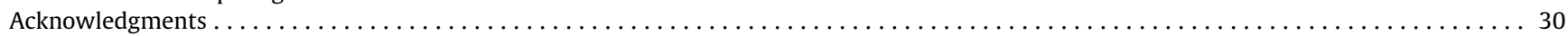

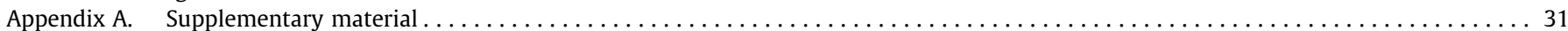

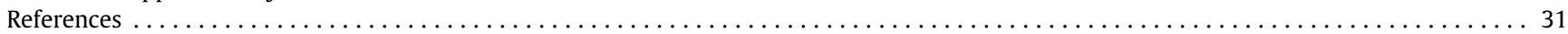

\section{Introduction}

Extracellular vesicles (EVs) are particles released by all cells and mediate a conserved form of intercellular communication [1]. Enclosed by one or more lipidic membranes, they consist of aqueous compartments which carry a vast array of biomolecules from the parental cell, such as lipids, proteins, various types of nucleic acids and soluble small molecules [2,3]. EVs have been observed among all kingdoms of life, from bacteria and archaea to mammals, highlighting their evolutionary importance [4]. In mammals, EVs are present in all biofluids such as blood, saliva, breast milk, urine, cerebrospinal fluid, amniotic fluid, semen and ascites [5-7].

\subsection{Types of EVs: exosomes, microvesicles and apoptotic bodies}

The term "extracellular vesicles" encompasses all secreted membrane vesicles from the cell, yet these vesicles appear particularly heterogeneous. As a matter of fact, during the past decade different subsets of EVs have been called exosomes, microvesicles, microparticles, ectosomes, oncosomes, apoptotic bodies, and multiple other names [8]. To simplify nomenclature, classes are defined based on their biogenesis. As a result, EVs have been divided into three main populations: exosomes, microvesicles and apoptotic bodies $[9,10]$. Exosomes are small vesicles $(30-120 \mathrm{~nm})$ formed and contained intracellularly inside multivesicular bodies (MVBs). Exosomes are released to the extracellular space through fusion of the MVB with the plasma membrane [10-12]. Microvesicles (50-1000 nm) are released from the cells by direct budding from the plasma membrane [12]. The last population of EVs are the apoptotic bodies (50-5000 nm), which are also released directly from the cell membrane but only by cells undergoing apoptosis $[10,13,14]$. The heterogeneity between EV populations, with overlapping sizes, and the lack of consensus on specific proteins that are unique to each EV subtype have greatly hindered their characterization at the subtype level, as well as the modification of subclass-specific properties and study of differences between subtype functions [15-18]. Nevertheless, in recent years the term "exosomes" has become increasingly popular in publications to refer to what is likely a mixture of different heterogeneous EV subtypes. In this review we will only use the term EVs.

\subsection{Functions of EVs}

EVs have a broad range of biological functions and participate in multiple physiological and pathological processes [19]. Their ability to mediate intercellular communication by transferring a wide spectrum of molecules between cells gives them an important role in complex biological processes like tumorigenesis [20], preparation of metastatic niches [21], elimination of cytotoxic drugs such as cisplatin [22], inflammation [23], immune response modulation 
[24], angiogenesis [25,26], tissue repair [27], apoptosis [28-30] and also in maintenance of homeostasis [31], amongst many others [32]. Since their composition reflects the parental cell status at the time of production, this makes them very attractive for the diagnostics field $[2,33]$. In addition, they are stable in many biological fluids and are relatively abundant, endowing EVs with plenty of potential as a reservoir of biomarkers. Liquid biopsies containing circulating EVs could allow monitoring of prognosis, progression of the disease and response to therapy in patients [2,33-35].

Furthermore, EVs are able to modulate cell phenotypes, differentiation and recruitment in a paracrine fashion [36]. As such, EVs possess similar therapeutic features as parental cells such as stem cells. However, EVs cannot self-replicate, hence potentially conferring a safer profile over stem cell transplantation in regenerative medicine [36-42]. Interestingly, EVs derived from biological fluids, such as plasma, also exert intrinsic bioactivities although the specific components are not always defined [43]. Given the capacity of EVs to effectively carry a broad variety of biological molecules through different biofluids with cellular specificity, EVs hold promise for drug delivery [3]. Taking it one step forward, an EVbased theranostic delivery platform has been recently proposed by loading both imaging tracers (for diagnosis) and therapeutic compounds (for delivery) into (or onto) EVs $[2,44]$. Taken together, EVs are emerging as a diagnostic toolbox, a new class of therapeutics, and a drug delivery vehicle (Fig. 1). All these potential applications are in the process of validation in many preclinical and clinical studies. In this review, we focus on their application as drug delivery systems (DDS) by systematically reviewing and analyzing the preclinical studies over the past decade (for earlier studies readers are recommended to an elegant review by Johnsen et al. [45]).

\section{Systematic review of EVs as drug delivery systems in preclinical studies}

\subsection{Introduction of the systematic review}

Having analyzed several aspects of EVs as DDS in comparison with the conventional liposomal DDS, we realize the urgency of a systematic review that offers an overview of the development of EVs as DDS, especially in the past decade when EVs have been extensively explored for drug delivery in various disease models (Fig. 2). In fact, for a comprehensive evaluation of any therapeutic delivery vehicle, it is crucial to test in detail the pharmacodynamics and the pharmacokinetics in preclinical models that resemble the human condition. In consequence, the choice of animal models based on the resemblance of their physiological features with the disease modus operandi, is of key importance to determine the translatability of the results into human therapies. Up to now, only mice, rats and zebrafish have been used in published preclinical studies of EVs as DDS. Pigs have been used for preclinical testing of EVs as therapeutics per se but not as DDS [46,47]. There are no published records to investigate EVs, either as therapeutics per se or as DDS, in non-human primates, which are generally used as a model in the final preclinical stages prior to human clinical trials. Another factor to take into account for the development of DDS is the drug-encapsulation efficiency, which can be relatively high for liposomal drug formulations [48]. For EVs, availability of drug loading strategies and efficiencies is limited as they are biological products derived from cellular activity that offer less freedom to adapt their composition of lipid membranes and interior in comparison to liposomal delivery systems. There are two main

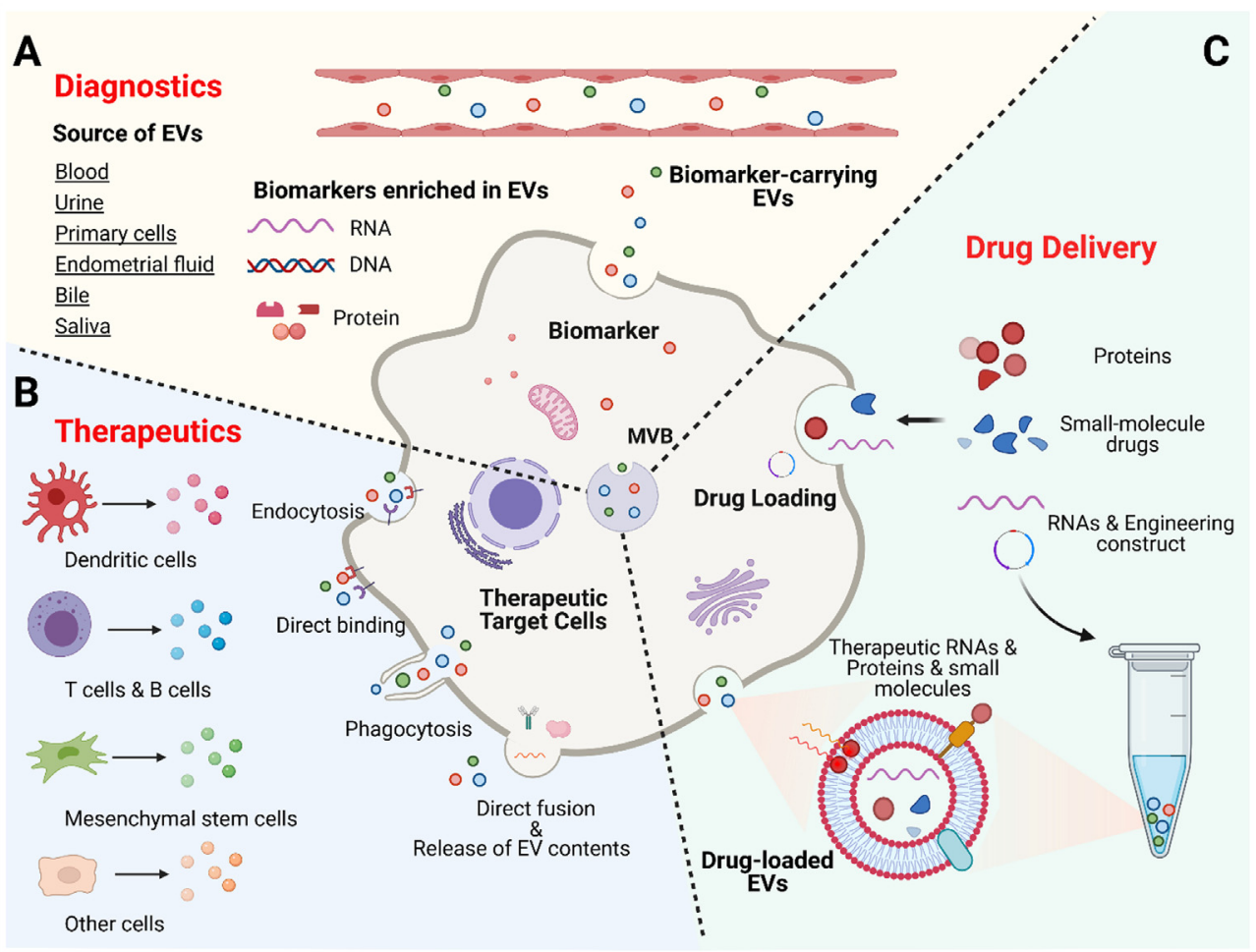

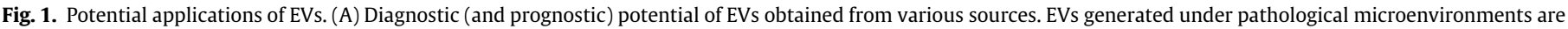

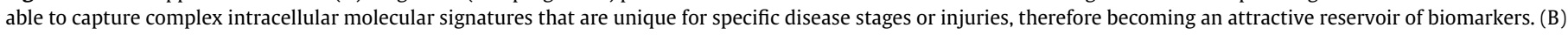

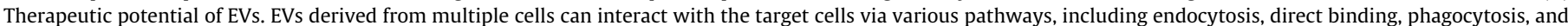

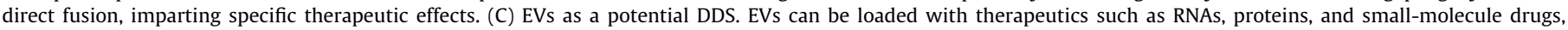
delivering these cargoes to target cells. 

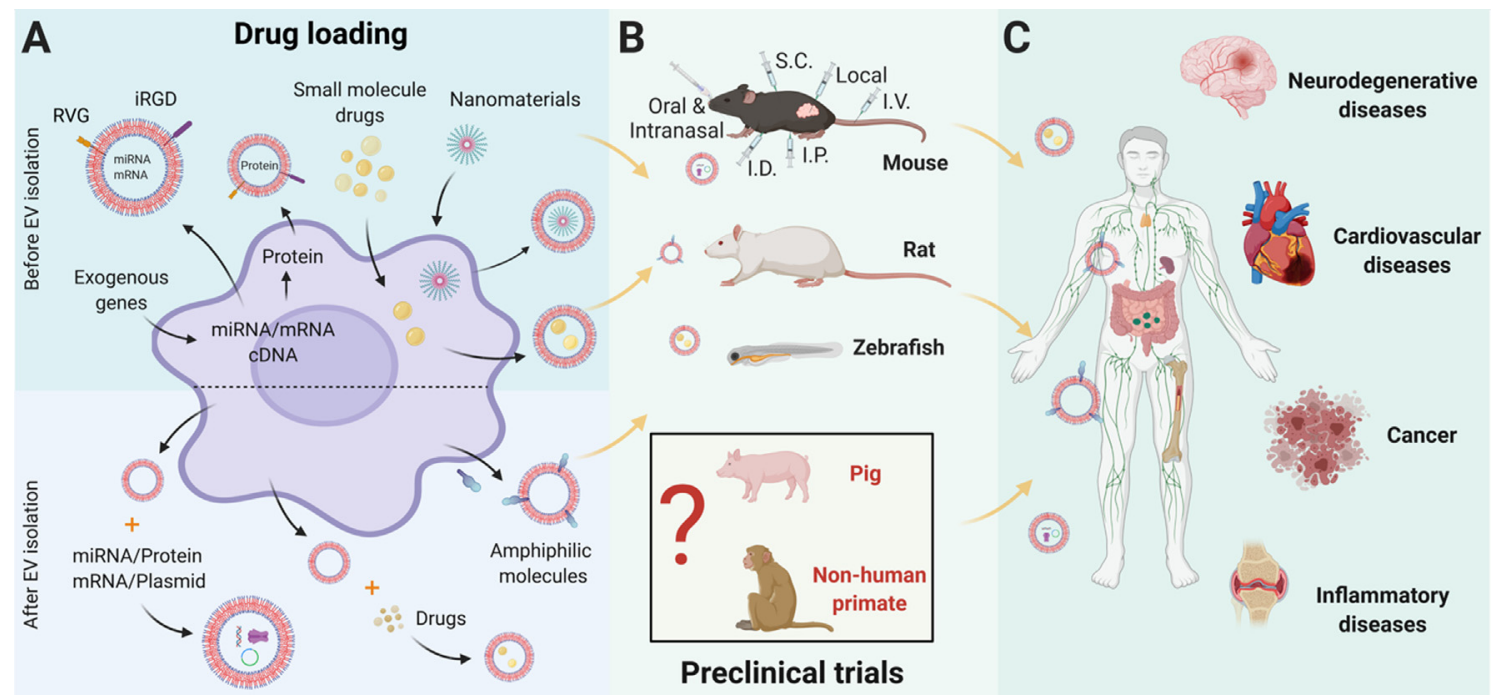

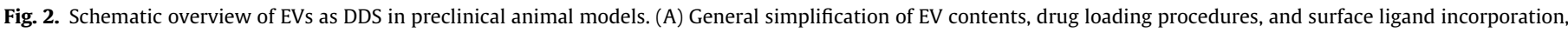

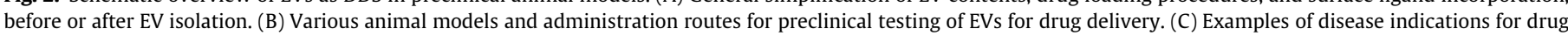
delivery via EVs.

strategies for loading drugs into EVs: A) Before EV isolation, drugs are loaded by addition to and manipulation of the EV donor cells. This strategy demands compatibility and suitability of the parental cells with the drugs to encapsulate in EVs. B) Drugs are loaded after $\mathrm{EV}$ isolation. This strategy requires preservation of the structure and functionality of the vesicles [49]. In addition, the routes of administration, size, charge and surface modifications of EVs are influencing the pharmacokinetics and pharmacodynamics of drug-loaded EVs. In Fig. 2, we provide an overview of the parameters, including payloads, drug loading strategies, EV surface modifications, administration routes, animal models and disease indications, that have been considered in the experimental design of preclinical testing of EVs as DDS.

In this article, we will assess the performance of EVs as DDS in preclinical models in the form of a systematic review. For each study published in the past decade, disease, animal model, EV donor cell type, active pharmaceutical ingredient (API) loaded, EV surface modifications, API loading procedure, EV size and charge, estimation of EV purity, presence of biodistribution studies and administration route were qualitatively analyzed in a defined and reproducible way. After analysis of the performance of EVs as DDS in comparison with liposomes, we interpret the trends observed for the past decade and try to define the niches where to apply EVs in the future.

\subsection{Approaches for systematic review}

The main goal of this systematic review is to comprehensively analyze and interpret all the literature from the past decade on the preclinical testing of EVs as a DDS with a non-biased, precise and reproducible approach, following the principles defined in the Preferred Reporting Items for Systematic Reviews and Metaanalysis (PRISMA) statement [50] and the Cochrane Handbook for Systematic Reviews of Interventions [51]. The search parameters and criteria employed (Table 1) were defined based on a consensus between multiple investigators (P.E.M., L.T., C.H., G.S., R.S., and J.W.W.) using exclusively PubMed as a database.

\subsection{Inclusion and exclusion criteria}

As we intended to carry out a systematic review of the preclinical status of EVs as DDS during the past decade, we only included journal articles published in English from the 1st of January 2010 until the 1st of January 2021. In this manner we aimed to provide an overview of the recent development in the field. We focus on original research articles, while excluding all other types of publications such as opinion articles, case reports, and editorials. Moreover, as this review provides an overview of the preclinical status of EVs as DDS, the PubMed option "Other animals" was applied to the species filter. EVs were defined according to the Minimal Information for Studies of Extracellular Vesicles 2018 (MISEV2018) guidelines [8], excluding synthetic nanoparticles and exosomemimetic nanovesicles. Regarding the selection of keywords for our search we set a criterion that the articles of potential interest should contain in their titles or abstracts at least one term of each of the 4 categories that were defined in the Keywords section of Table 1. Furthermore, "EVs as drug delivery systems" in this study were defined as EVs that were purposely used for loading and delivery of therapeutic molecules. Enriched EV fractions containing endogenous molecules which were not introduced for a delivery purpose were excluded from our systematic review.

Articles that did not meet all the selection criteria were excluded for analysis. Additionally, to increase the power and the sensitivity of our search, we performed a subsequent PubMed search with the same search keywords format as described in Table 1 but opted for Reviews and Systematic Reviews instead of Journal Articles and excluded the filter Other Animals. The aim of this additional search was to screen the resulting reviews for

Table 1

Eligibility criteria for this systematic review.

\begin{tabular}{ll}
\hline Publication & \\
\hline Database & PubMed \\
Language & English \\
Time period & $01 / 01 / 2010-01 / 01 / 2021$ \\
Publication type & Journal Article \\
Species & Other Animals \\
Keywords [Title/ & extracellular vesicles / exosomes / microvesicles / \\
\multicolumn{1}{c}{ Abstract] } & apoptotic bodies / microparticles \\
& drug / therapeutic / small molecule / antioxidant / anti- \\
& inflammatory / chemotherapeutic / silencing / siRNA / \\
& miRNA / mRNA / plasmid / kinase inhibitor \\
& animal / mice / mouse / murine / rats / rat / pig / \\
& zebrafish / primate / monkey / chimpanzee \\
& in vivo / preclinical \\
\hline &
\end{tabular}


analytical tables or figures which referenced journal articles that fulfilled our criteria but that we might have missed with our previous search parameters. Explicit search parameters are provided in the Supplementary Materials Section A. Any studies that generated eligibility doubts were brought to and resolved by J.W.W., R. S. and G.S. The workflow for this Systematic Review is presented in Fig. 3.

\section{Data analysis of systematic review}

\subsection{Categorization of selected publications}

In this systematic review, we categorized the selected 157 publications, based on disease or pathogenic conditions where EVs were investigated as DDS, into 5 groups: cancer (Table 2a), cardiovascular disease (Table $2 \mathrm{~b}$ ), neurological disease (Table 2c), inflammatory disease (Table $2 \mathrm{~d}$ ) and other diseases (Table 2e). All the tables were designed with ten categories (disease/condition tested, animal model, EV donor cell type, API loaded, EV surface modifications, API loading method, EV size \& charge, EV purity analysis, biodistribution studies and administration route). EV isolation issues have been exhaustedly reviewed in the literature $[2,8,12,52-55]$ and therefore were not included in the current study. We carried out a systematic analysis of the approaches and trends in the field for the past decade in a rigorous and unbiased manner. The detailed criteria employed for data analysis can be found in Supplementary Materials Section B.

\subsection{Diving deeper into the tables: Analytical interpretation}

\subsubsection{Diseases}

The main tables of this systematic review were grouped by disease categories of the study (Tables $2 a-2 e$ ) and then the table contents were organized by specific diseases/conditions, to facilitate the search of study parameters by readers who are interested in one particular disease or disease category. As shown in Fig. 4a and Table 2a, the majority (66.2\%) of publications on EVs for drug delivery during the past decade were focused on cancer treatment. $70.6 \%$ of total studies in the year of 2020 were cancer related (Fig. $4 \mathrm{~b}$ ), of which $27 \%$ were on breast cancer, $18 \%$ on lung cancer and $12 \%$ on brain cancer. The extensive studies of EVs in cancer- related preclinical models may be partly attributable to the relatively easy adoption and readily availability of tumor animal models. Studies on cardiovascular disease (12.7\%), the second most investigated disease type, were essentially represented by myocardial infarction and stroke (Table 2b, Fig. 4a). Neurological and inflammatory disease studies constitute $6.4 \%$ and $5.7 \%$ of the total, respectively, (Fig. 4a) where the majority of publications were on Parkinson's and Alzheimer's disease (in the neurological class) and arthritis (for the inflammatory diseases category) (Table 2c, Table $2 \mathrm{~d}$ ). In the "other" disease group (10.2\%), we categorized a variety of liver, kidney, muscular and infectious diseases together with diabetes, obesity, immunomodulation and wound healing studies.

\subsubsection{Animal models}

With regard to the animal models used (Fig. 5a), a vast majority of publications used mice $(87.3 \%)$, followed by rats $(11.5 \%)$ and zebrafish (1.3\%). The overwhelming majority of studies using mice has been increasing over time (Fig. $5 \mathrm{~b}$ ). The extensive usage of mice as experimental animal model is mainly because of historic preferences and available data from related studies as well as cost-effectiveness. These percentages though, are different between disease groups (Table 2a). Rats represent 55\% and 40\% of animal studies for inflammatory and cardiovascular diseases, respectively. In the case of cardiovascular diseases rats have been the main animal model for decades, where detailed and effective experimental procedures for multiple conditions, like myocardial infarction and stroke are available as well as rat strains that spontaneously develop these diseases [213]. A similar argument applies to inflammatory diseases [214]. The two publications using zebrafish, were focused on treating brain cancer (Table 2a) as zebrafish represents a model that might gain popularity due to its costeffective maintenance and the opportunity to dynamically visualize tumor growth in vivo [215].

\subsubsection{Donor cell types}

Based on the origin of EVs, we grouped the EV donor cell types into eight different categories (Fig. 6a,b). Among all the analyzed preclinical studies, EVs derived from cancer cell lines (23.6\%), stem cells (22.9\%), and HEK293 cells (21.7\%) were most commonly used. Interestingly, in the past five years, a few studies explored the drug

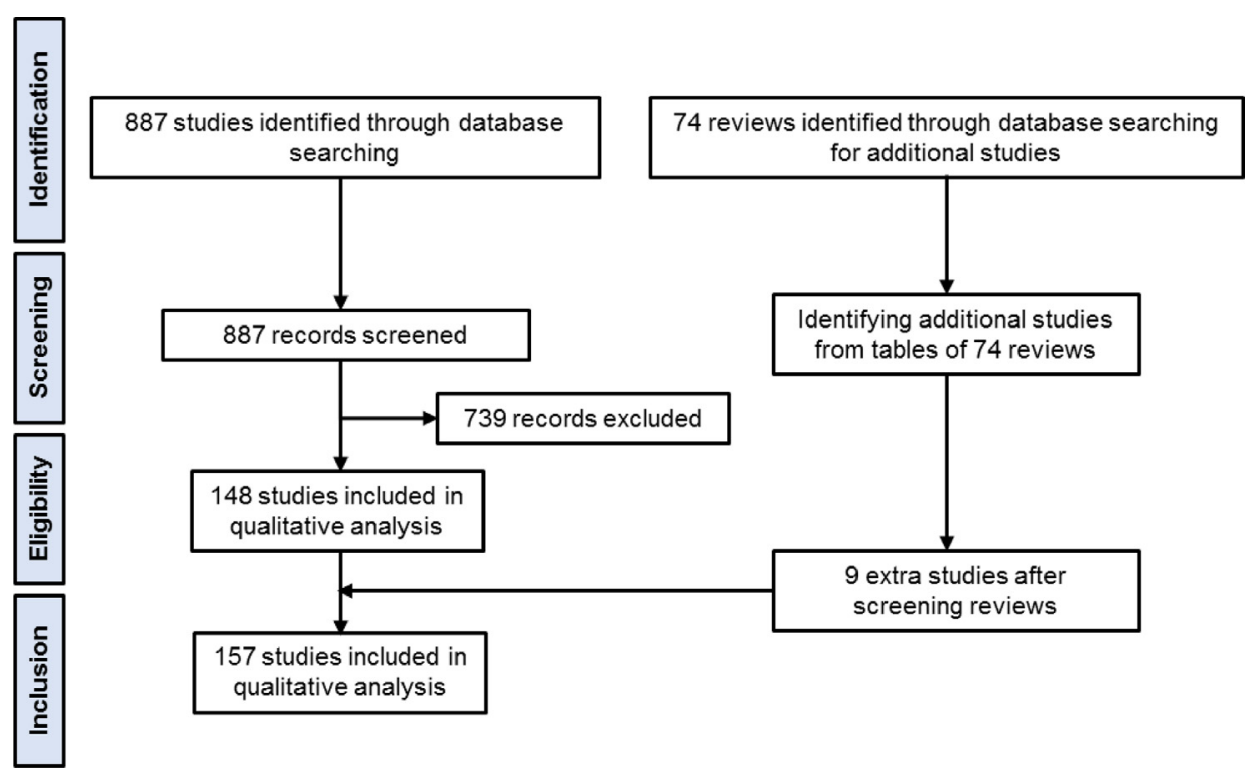

Fig. 3. Flowchart diagram of the systematic review according to the PRISMA statement. 
Table 2a

Preclinical studies of EVs as drug delivery systems for cancer treatment.

\begin{tabular}{|c|c|c|c|c|c|c|c|c|c|c|c|}
\hline Disease/condition & $\begin{array}{l}\text { Animal } \\
\text { model }\end{array}$ & $\begin{array}{l}\text { Donor cell } \\
\text { type }\end{array}$ & $\begin{array}{l}\text { Active } \\
\text { pharmaceutical } \\
\text { ingredient (API) }\end{array}$ & Surface modifications & $\begin{array}{l}\text { API loading } \\
\text { procedure }\end{array}$ & Size/charge* & $\begin{array}{l}\text { Purity } \\
\text { estimation }\end{array}$ & $\begin{array}{l}\text { Biodistribution } \\
\text { studies }\end{array}$ & $\begin{array}{l}\text { Route of } \\
\text { administration }\end{array}$ & $\begin{array}{l}\text { Year of } \\
\text { publication }\end{array}$ & Ref. \\
\hline \multicolumn{12}{|l|}{ Brain cancer } \\
\hline Glioma & Mouse & BMSCs & $\begin{array}{l}\text { Indocyanine green \& } \\
\text { Curcumin }\end{array}$ & None & $\begin{array}{l}\text { After isolation EVs, } \\
\text { Physical } \\
\text { (Electroporation) }\end{array}$ & $\begin{array}{l}160 \mathrm{~nm}(\mathrm{DLS}) / \\
-16 \mathrm{mV}\end{array}$ & No & Yes & I.V. & 2020 & [56] \\
\hline Glioma & Mouse & $\begin{array}{l}\text { MSCs, DCs \& } \\
\text { HEK293T }\end{array}$ & PTEN-mRNA & None & $\begin{array}{l}\text { Before EVs isolation, } \\
\text { Transfection based } \\
\text { (Plasmid) }\end{array}$ & $70-110 \mathrm{~nm}(\mathrm{DLS})$ & No & Yes & I.V. & 2020 & [57] \\
\hline Glioma & Mouse & $\begin{array}{l}\text { RAW264.7 } \\
\text { Macrophage }\end{array}$ & $\begin{array}{l}\text { Curcumin \& } \\
\text { superparamagnetic } \\
\text { iron oxide } \\
\text { nanoparticles }\end{array}$ & $\begin{array}{l}\text { Neuropilin-1- } \\
\text { targeted peptide }\end{array}$ & $\begin{array}{l}\text { After isolation EVs, } \\
\text { physical } \\
\text { (Incubation) }\end{array}$ & $\begin{array}{l}122.7 \pm 6.5 \mathrm{~nm} \\
(\mathrm{NTA}) / \\
-24.1 \pm 2.2 \mathrm{mV}\end{array}$ & No & Yes & I.V. & 2018 & [58] \\
\hline Glioma & Mouse & MSCs & miRNA-124a & None & $\begin{array}{l}\text { Before EVs isolation, } \\
\text { Transfection based } \\
\text { (Lentivirus) }\end{array}$ & $100-125 \mathrm{~nm}$ (NTA) & No & No & I.P. & 2018 & [59] \\
\hline Glioblastoma & Rat & HEK293T & Anti-miR-21 & T7 peptide & $\begin{array}{l}\text { After isolation EVs, } \\
\text { Physical } \\
\text { (Electroporation) }\end{array}$ & $\begin{array}{l}15-50 \mathrm{~nm} \text { (DLS) / } \\
-10 \text { to }-3 \mathrm{mV}\end{array}$ & No & Yes & I.V. & 2020 & [60] \\
\hline Glioblastoma & Mouse & $\begin{array}{l}\text { Malignant } \\
\text { cells }\end{array}$ & CRISPR/Cas9 & TNF- $\alpha$ & $\begin{array}{l}\text { After isolation EVs, } \\
\text { Physical } \\
\text { (Electroporation) }\end{array}$ & NA & No & No & I.V. & 2019 & [61] \\
\hline Glioblastoma & Mouse & $\begin{array}{l}\text { Embryonic } \\
\text { stem cells }\end{array}$ & Paclitaxel & cRGD & $\begin{array}{l}\text { After isolation EVs, } \\
\text { Physical } \\
\text { (Incubation) }\end{array}$ & $\begin{array}{l}125 \pm 27 \mathrm{~nm} \\
\text { (NanoFCM) }\end{array}$ & No & Yes & I.V. & 2019 & [62] \\
\hline Glioblastoma & Rat & HEK-293 T & miRNA-21-Sponge & None & $\begin{array}{l}\text { Before EVs isolation, } \\
\text { Transfection based } \\
\text { (Plasmid) }\end{array}$ & $\begin{array}{l}66.65 \pm 39.88 \mathrm{~nm} \\
\text { PDI } 0.317 \text { (DLS) }\end{array}$ & No & No & Local & 2019 & [63] \\
\hline Glioblastoma & Mouse & HEK293T & $\begin{array}{l}\text { CD-UPRT mRNA \& } \\
\text { protein }\end{array}$ & None & $\begin{array}{l}\text { Before EVs isolation, } \\
\text { Transfection based } \\
\text { (Plasmid) }\end{array}$ & 88-152 nm (NTA) & No & No & Local & 2017 & [64] \\
\hline $\begin{array}{l}\text { Glioblastoma- } \\
\text { astrocytoma }\end{array}$ & Zebrafish & bEND.3 cells & SiVEGF & None & $\begin{array}{l}\text { After isolation EVs, } \\
\text { Chemical } \\
\text { (Lipofetamine }^{\circledast} \\
2000 \text { transfection) }\end{array}$ & NA & No & No & I.V. & 2017 & [65] \\
\hline $\begin{array}{l}\text { Glioblastoma- } \\
\text { astrocytoma }\end{array}$ & Zebrafish & $\begin{array}{l}\text { U-87 MG, } \\
\text { bEND.3, } \\
\text { PFSK-1 \& A- } \\
172\end{array}$ & $\begin{array}{l}\text { Rhodamine 123, } \\
\text { Paclitaxel \& } \\
\text { Doxorubicin }\end{array}$ & None & $\begin{array}{l}\text { After isolation EVs, } \\
\text { Physical } \\
\text { (Incubation) }\end{array}$ & 30-100 nm (DLS) & No & Yes & I.V. & 2015 & [66] \\
\hline $\begin{array}{l}\text { Glioblastoma } \\
\text { multiforme }\end{array}$ & Mouse & L929 cells & $\begin{array}{l}\text { Methotrexate \& KLA } \\
\text { peptide }\end{array}$ & LDL \& KLA peptide & $\begin{array}{l}\text { Before EVs isolation, } \\
\text { Ultraviolet } \\
\text { irradiation }\end{array}$ & $\begin{array}{l}318.3 \pm 15.5 \mathrm{~nm} \\
\text { (DLS) / about } \\
-10 \mathrm{mV}\end{array}$ & No & Yes & I.V. & 2018 & [67] \\
\hline $\begin{array}{l}\text { Glioblastoma } \\
\text { multiforme }\end{array}$ & Rat & MSCs & miRNA-146b & None & $\begin{array}{l}\text { Before EVs isolation, } \\
\text { Transfection based } \\
\text { (Plasmid) }\end{array}$ & $\mathrm{NA}$ & No & No & Local & 2013 & [68] \\
\hline \multicolumn{12}{|l|}{ Breast cancer } \\
\hline & IVIouse & ПЕК29נו & \& Doxorubicin & hyaluronidase & $\begin{array}{l}\text { (Plasmid); After } \\
\text { isolation EVs, } \\
\text { Physical (Incubation } \\
\text { \& electroporation) }\end{array}$ & (DLS) & No & res & Luld1 & 2021 & {$[03]$} \\
\hline Breast cancer & Mouse & $\begin{array}{l}\text { HEK293T } \\
\text { (Expi293) }\end{array}$ & $\begin{array}{l}\text { AntiCD3 \& antiHer2 } \\
\text { antibody }\end{array}$ & $\begin{array}{l}\text { AntiCD3 \& antiHer2 } \\
\text { antibody }\end{array}$ & $\begin{array}{l}\text { Before EVs isolation, } \\
\text { Transfection based } \\
\text { (Plasmid) }\end{array}$ & $109 \mathrm{~nm}$ (NTA) & Yes & No & I.V. & 2020 & [70] \\
\hline
\end{tabular}




\begin{tabular}{|c|c|c|c|c|c|c|c|c|c|c|c|}
\hline Disease/condition & $\begin{array}{l}\text { Animal } \\
\text { model }\end{array}$ & $\begin{array}{l}\text { Donor cell } \\
\text { type }\end{array}$ & $\begin{array}{l}\text { Active } \\
\text { pharmaceutical } \\
\text { ingredient (API) }\end{array}$ & Surface modifications & $\begin{array}{l}\text { API loading } \\
\text { procedure }\end{array}$ & Size/charge ${ }^{*}$ & $\begin{array}{l}\text { Purity } \\
\text { estimation }\end{array}$ & $\begin{array}{l}\text { Biodistribution } \\
\text { studies }\end{array}$ & $\begin{array}{l}\text { Route of } \\
\text { administration }\end{array}$ & $\begin{array}{l}\text { Year of } \\
\text { publication }\end{array}$ & Ref. \\
\hline Breast cancer & Mouse & $\begin{array}{l}\text { RAW264.7 } \\
\text { Macrophage }\end{array}$ & $\begin{array}{l}\text { Paclitaxel \& } \\
\text { Doxorubicin }\end{array}$ & None & $\begin{array}{l}\text { After isolation EVs, } \\
\text { Physical } \\
\text { (Incubation, } \\
\text { sonication \& } \\
\text { exclusion } \\
\text { chromatography) }\end{array}$ & $\begin{array}{l}\text { Dox-EV } \\
162.1 \pm 5.5 \mathrm{~nm} \& \\
\text { PTX-EV } \\
129.4 \pm 2.3 \mathrm{~nm} \\
\text { (NTA) }\end{array}$ & No & No & I.V. & 2020 & [71] \\
\hline Breast cancer & Mouse & $4 \mathrm{~T} 1$ cells & $\begin{array}{l}\text { TK-NTR-encoding } \\
\text { minicircle DNA }\end{array}$ & None & $\begin{array}{l}\text { Before EVs isolation, } \\
\text { Transfection based } \\
\text { (Plasmid) }\end{array}$ & $136-160 \mathrm{~nm}$ (NTA) & No & No & Local & 2019 & [72] \\
\hline Breast cancer & Mouse & BMSCs & Doxorubicin & DARPin & $\begin{array}{l}\text { After isolation EVs, } \\
\text { Physical } \\
\text { (Electroporation) }\end{array}$ & $120 \mathrm{~nm}$ (DLS) & No & Yes & I.V. & 2019 & [73] \\
\hline Breast cancer & Mouse & Blood & $\begin{array}{l}\text { Chimeric peptide } \\
\text { (ChiP) }\end{array}$ & $\begin{array}{l}\text { Chimeric peptide } \\
\text { (ChiP) }\end{array}$ & $\begin{array}{l}\text { After isolation EVs, } \\
\text { Physical (Incubation } \\
\text { on ice) }\end{array}$ & $\begin{array}{l}132.6 \mathrm{~nm}, \mathrm{PDI} \\
0.306 \text { (DLS) }\end{array}$ & No & Yes & I.V. & 2019 & [74] \\
\hline Breast cancer & Mouse & MSCs & Paclitaxel & None & $\begin{array}{l}\text { Before EVs isolation, } \\
\text { Incubation }\end{array}$ & $\begin{array}{l}204 \pm 93.1 \mathrm{~nm} \\
(\mathrm{NTA}) / \\
-43.08 \pm 1.58 \mathrm{mV}\end{array}$ & No & No & I.V. & 2019 & [75] \\
\hline Breast cancer & Mouse & $\begin{array}{l}\text { M1- } \\
\text { polarized } \\
\text { macrophages }\end{array}$ & Paclitaxel & None & $\begin{array}{l}\text { After isolation EVs, } \\
\text { Physical } \\
\text { (Sonication) }\end{array}$ & $\begin{array}{l}172.8 \mathrm{~nm} \\
\text { (DLS\&NTA)/ } \\
-12 \mathrm{mV}\end{array}$ & No & Yes & I.V. & 2019 & [76] \\
\hline Breast cancer & Mouse & $4 \mathrm{~T} 1$ cells & $\begin{array}{l}\text { Sinoporphyrin } \\
\text { sodium }\end{array}$ & Sinoporphyrin sodium & $\begin{array}{l}\text { After isolation EVs, } \\
\text { Physical } \\
\text { (Incubation) }\end{array}$ & $\begin{array}{l}126.71 \pm 3.86, \text { PDI } \\
0.18 \pm 0.05 \\
\text { (DLS\&NA) / } \\
-10.67 \pm 0.52 \mathrm{mV}\end{array}$ & No & Yes & I.V. & 2019 & [77] \\
\hline Breast cancer & Mouse & $\begin{array}{l}\text { H22 \& } \\
\text { Bel7402 cells }\end{array}$ & Doxorubicin & None & $\begin{array}{l}\text { Before EVs isolation, } \\
\text { Transfection based } \\
\text { (Plasmid) }\end{array}$ & $\begin{array}{l}260 \pm 15 \mathrm{~nm}, \text { PDI } \\
0.145 \pm 0.032 \\
\text { (DLS) / } \\
-11.0 \pm 0.4 \mathrm{mV}\end{array}$ & No & Yes & I.V. & 2019 & [78] \\
\hline Breast cancer & Mouse & MSCs & miRNA-142-3p & None & $\begin{array}{l}\text { After isolation EVs, } \\
\text { Physical } \\
\text { (Electroporation) }\end{array}$ & $103 \mathrm{~nm}$ (DLS) & No & Yes & I.V. & 2018 & [79] \\
\hline Breast cancer & Mouse & HEK293T & siSurvivin & $\begin{array}{l}\text { Folate, PSMA RNA } \\
\text { aptamer \& EGFR RNA } \\
\text { aptamer (All } \\
\text { conjugated to 3WJ) }\end{array}$ & $\begin{array}{l}\text { After isolation EVs, } \\
\text { Chemical (ExoFect } \\
\text { Exosome } \\
\text { transfection kit) }\end{array}$ & $\begin{array}{l}103-120 \mathrm{~nm}(\mathrm{NTA}) \\
/-15.6 \pm 27.9 \mathrm{mV}\end{array}$ & Yes & Yes & I.V. & 2018 & [80] \\
\hline Breast cancer & Mouse & $\begin{array}{l}\text { Dendritic } \\
\text { cells }\end{array}$ & Paclitaxel & $\begin{array}{l}\text { AS1411 aptamer } \\
\text { conjugated to } \\
\text { cholesterol-PEG }\end{array}$ & $\begin{array}{l}\text { After isolation EVs, } \\
\text { Physical } \\
\text { (Sonication) }\end{array}$ & $\begin{array}{l}111 \mathrm{~nm}(\mathrm{NTA}) / \\
-25.6 \mathrm{mV}\end{array}$ & No & Yes & I.V. & 2018 & [81] \\
\hline Breast cancer & Mouse & НЕК293 & HchrR6 mRNA & $\begin{array}{l}\text { LS-ML39-C1-C2-His } \\
\text { (EVHB) }\end{array}$ & $\begin{array}{l}\text { Before EVs isolation, } \\
\text { Transfection based } \\
\text { (Plasmid) }\end{array}$ & $30-100 \mathrm{~nm}(\mathrm{NTA})$ & No & No & I.P. & 2018 & [82] \\
\hline Breast cancer & Mouse & HEK293 & $\begin{array}{l}\text { PH20 hyaluronidase } \\
\text { \& Doxorubicin }\end{array}$ & PH20 hyaluronidase & $\begin{array}{l}\text { Before EVs isolation, } \\
\text { Transfection based } \\
\text { (Plasmid); After } \\
\text { isolation EVs, } \\
\text { Physical } \\
\text { (Incubation) }\end{array}$ & $95 \mathrm{~nm}$ (DLS) & No & Yes & Local & 2018 & [83] \\
\hline Breast cancer & Mouse & $\begin{array}{l}\text { Human red } \\
\text { blood cells }\end{array}$ & $\begin{array}{l}\text { Anti-miR-125b, Cas9 } \\
\text { mRNA, \& guide RNAs }\end{array}$ & None & $\begin{array}{l}\text { After isolation EVs, } \\
\text { Physical } \\
\text { (Electroporation) }\end{array}$ & $\begin{array}{l}\text { About } 140 \mathrm{~nm}, \text { PDI } \\
0.07 \text { (NTA\&DLS) / } \\
-11.5 \mathrm{mV}\end{array}$ & No & Yes & I.P. \& Local & 2018 & [84] \\
\hline Breast cancer & Mouse & $\begin{array}{l}\text { Dendritic } \\
\text { cells }\end{array}$ & $\begin{array}{l}\text { miRNA let-7 \& } \\
\text { siRNA-VEGF }\end{array}$ & AS1411 aptamer & $\begin{array}{l}\text { After isolation EVs, } \\
\text { Physical } \\
\text { (Electroporation) }\end{array}$ & $\begin{array}{l}77 \mathrm{~nm} \text { (NTA) / } \\
-16.4 \mathrm{mV}\end{array}$ & No & Yes & I.V. & 2017 & [85] \\
\hline
\end{tabular}




\begin{tabular}{|c|c|c|c|c|c|c|c|c|c|c|c|}
\hline Disease/condition & $\begin{array}{l}\text { Animal } \\
\text { model }\end{array}$ & $\begin{array}{l}\text { Donor cell } \\
\text { type }\end{array}$ & $\begin{array}{l}\text { Active } \\
\text { pharmaceutical } \\
\text { ingredient (API) }\end{array}$ & Surface modifications & $\begin{array}{l}\text { API loading } \\
\text { procedure }\end{array}$ & Size/charge ${ }^{*}$ & $\begin{array}{l}\text { Purity } \\
\text { estimation }\end{array}$ & $\begin{array}{l}\text { Biodistribution } \\
\text { studies }\end{array}$ & $\begin{array}{l}\text { Route of } \\
\text { administration }\end{array}$ & $\begin{array}{l}\text { Year of } \\
\text { publication }\end{array}$ & Ref. \\
\hline Breast cancer & Mouse & $\begin{array}{l}\text { MDA-MB- } \\
231 \& \text { STOSE }\end{array}$ & Doxorubicin & None & $\begin{array}{l}\text { After isolation EVs, } \\
\text { Physical } \\
\text { (Electroporation) }\end{array}$ & $101 \mathrm{~nm}(\mathrm{NTA})$ & No & No & I.P. & 2016 & [86] \\
\hline Breast cancer & Mouse & $\begin{array}{l}\text { MDA-MB- } \\
231 \& \text { HCT- } \\
116\end{array}$ & Doxorubicin & None & $\begin{array}{l}\text { After isolation EVs, } \\
\text { Physical } \\
\text { (Electroporation) }\end{array}$ & $\begin{array}{l}176 \pm 53 \mathrm{~nm}, \\
209 \pm 54 \mathrm{~nm}, \\
\text { respectively (NTA) }\end{array}$ & No & Yes & I.V. & 2015 & [87] \\
\hline Breast cancer & Mouse & MCF-7 cells & Doxorubicin & None & $\begin{array}{l}\text { Before EVs isolation, } \\
\text { Incubation }\end{array}$ & $40-100 \mathrm{~nm}($ TEM) & No & No & S.C. & 2015 & [88] \\
\hline Breast Cancer & Mouse & $\begin{array}{l}\text { Immature } \\
\text { dendritic } \\
\text { cells }\end{array}$ & Doxorubicin & $\begin{array}{l}\text { AlphaV integrin- } \\
\text { specific iRGD peptide }\end{array}$ & $\begin{array}{l}\text { After isolation EVs, } \\
\text { Physical } \\
\text { (Electroporation) }\end{array}$ & $93 \mathrm{~nm}$ (NTA) & No & Yes & I.V. & 2014 & [89] \\
\hline Breast cancer & Mouse & HEK293 & miRNA-let-7a & $\begin{array}{l}\text { Transmembrane } \\
\text { domain of platelet- } \\
\text { derived growth factor } \\
\text { receptor fused to GE11 } \\
\text { peptide }\end{array}$ & $\begin{array}{l}\text { Before EVs isolation, } \\
\text { Transfection based } \\
\text { (miRNA) }\end{array}$ & NA & No & Yes & I.V. & 2013 & [90] \\
\hline $\begin{array}{l}\text { Breast cancer } \\
\text { multidrug } \\
\text { resistance }\end{array}$ & Mouse & HEK293T & Doxorubicin & $\begin{array}{l}\text { Lipidomimetic chains- } \\
\text { grafted hyaluronic } \\
\text { acid }\end{array}$ & $\begin{array}{l}\text { Before EVs isolation, } \\
\text { Ultraviolet } \\
\text { irradiation }\end{array}$ & $\begin{array}{l}449.1 \pm 15.1 \mathrm{~nm}, \\
\text { PDI } 0.29 \pm 0.02 \\
(D L S)\end{array}$ & No & Yes & I.V. & 2019 & [91] \\
\hline $\begin{array}{l}\text { Breast cancer with } \\
\text { Lung metastasis }\end{array}$ & Mouse & $\begin{array}{l}\text { Murine } \\
\text { macrophage }\end{array}$ & $\begin{array}{l}\text { Laurate } \\
\text { functionalized Pt } \\
\text { (IV) prodrug }\end{array}$ & None & $\begin{array}{l}\text { After isolation EVs, } \\
\text { Physical } \\
\text { (Incubation) }\end{array}$ & $\begin{array}{l}61.9 \pm 1.74 \mathrm{~nm}, \text { PDI } \\
0.168 \pm 0.021 \\
\text { (DLS) / - } \\
9.39 \pm 0.56 \mathrm{mV}\end{array}$ & No & Yes & I.V. & 2019 & [92] \\
\hline $\begin{array}{l}\text { Metastatic breast } \\
\text { cancer }\end{array}$ & Mouse & $\begin{array}{l}\text { HUVECS \& } \\
4 \text { T1 cells }\end{array}$ & siS100A4 & None & $\begin{array}{l}\text { After isolation EVs, } \\
\text { Physical (Incubation } \\
\text { \& extrusion) }\end{array}$ & $\begin{array}{l}263.71 \pm 24.84, \text { PDI } \\
0.32 \pm 0.01 \text { (DLS) / } \\
-28.63 \pm 0.33 \mathrm{mV}\end{array}$ & No & Yes & I.V. & 2020 & [93] \\
\hline $\begin{array}{l}\text { Hypoxic breast cancer } \\
\text { tumors }\end{array}$ & Mouse & $\begin{array}{l}\text { MDA-MB- } \\
231\end{array}$ & Olaparib & $\begin{array}{l}\text { SPIO } \\
\text { (superparamagnetic } \\
\text { iron oxide) } \\
\text { nanoparticles }\end{array}$ & $\begin{array}{l}\text { After isolation EVs, } \\
\text { Physical } \\
\text { (Electroporation) }\end{array}$ & $110-170 \mathrm{~nm}$ (NTA) & No & Yes & Local & 2018 & [94] \\
\hline $\begin{array}{l}\text { Triple-negative breast } \\
\text { cancer }\end{array}$ & Mouse & HEK293T & PH20 hyaluronidase & PH20 hyaluronidase & $\begin{array}{l}\text { Before EVs isolation, } \\
\text { Transfection based } \\
\text { (Plasmid) }\end{array}$ & $\begin{array}{l}\text { About } 100 \mathrm{~nm} \\
\text { (DLS) }\end{array}$ & No & No & Local & 2019 & [95] \\
\hline $\begin{array}{l}\text { Triple-negative breast } \\
\text { cancer }\end{array}$ & Mouse & Macrophages & $\begin{array}{l}\text { Doxorubicin \& } \\
\text { cholesterol-modified } \\
\text { miRNA-159 }\end{array}$ & $\begin{array}{l}\text { Disintegrin and } \\
\text { metalloproteinase } 15 \\
\text { (A15) }\end{array}$ & $\begin{array}{l}\text { After isolation EVs, } \\
\text { Chemical (Mixing } \\
\text { with } \\
\text { trimethylamine } \\
\text { solution); After } \\
\text { isolation EVs, } \\
\text { Physical } \\
\text { (Incubation) }\end{array}$ & $\begin{array}{l}94.1 \pm 104.4 \mathrm{~nm} \\
\left(\text { empty }{ }^{\#} \text { A15- }\right. \\
\text { exosome, NTA) / } \\
-14.67 \pm 1.53 \mathrm{mV} \\
\text { (miRNA loaded) }\end{array}$ & No & Yes & I.V. & 2019 & [96] \\
\hline \multicolumn{12}{|l|}{ Colorectal cancer } \\
\hline Colorectal cancer & Mouse & MSCs & Doxorubicin & MUC1 aptamer & $\begin{array}{l}\text { After isolation EVs, } \\
\text { Physical } \\
\text { (Electroporation } \\
\text { method } \\
\text { (DOX@exosome)) }\end{array}$ & $\begin{array}{l}120 \pm 12 \mathrm{~nm}, \text { PDI } \\
0.5 \pm 0.02(\mathrm{DLS}) / \\
-80 \pm 12 \mathrm{mV}\end{array}$ & No & Yes & I.V. & 2020 & [97] \\
\hline Colorectal cancer & Mouse & HEK293T & si-ciRS-122 & None & $\begin{array}{l}\text { Before EVs isolation, } \\
\text { Transfection based } \\
\text { (Plasmid) }\end{array}$ & $\begin{array}{l}\text { About } 100 \mathrm{~nm} \\
\text { (NTA) }\end{array}$ & No & No & I.V. & 2020 & [98] \\
\hline
\end{tabular}




\begin{tabular}{|c|c|c|c|c|c|c|c|c|c|c|c|}
\hline Disease/condition & $\begin{array}{l}\text { Animal } \\
\text { model }\end{array}$ & $\begin{array}{l}\text { Donor cell } \\
\text { type }\end{array}$ & $\begin{array}{l}\text { Active } \\
\text { pharmaceutical } \\
\text { ingredient (API) }\end{array}$ & Surface modifications & $\begin{array}{l}\text { API loading } \\
\text { procedure }\end{array}$ & Size/charge ${ }^{*}$ & $\begin{array}{l}\text { Purity } \\
\text { estimation }\end{array}$ & $\begin{array}{l}\text { Biodistribution } \\
\text { studies }\end{array}$ & $\begin{array}{l}\text { Route of } \\
\text { administration }\end{array}$ & $\begin{array}{l}\text { Year of } \\
\text { publication }\end{array}$ & Ref. \\
\hline Colorectal cancer & Mouse & HEK293T & siSur-A647 \& Folate & Folic acid & $\begin{array}{l}\text { After isolation EVs, } \\
\text { Chemical (ExoFect } \\
\text { exosomes } \\
\text { transfection kit); } \\
\text { After isolation EVs, } \\
\text { Physical (Heat- } \\
\text { shock) }\end{array}$ & $136.5 \pm 3.5(\mathrm{NTA})$ & No & No & I.V. & 2019 & [99] \\
\hline Colorectal cancer & Mouse & $\begin{array}{l}\text { LIM1215 } \\
\text { cells }\end{array}$ & Doxorubicin & A33Ab-US & $\begin{array}{l}\text { After isolation EVs, } \\
\text { Physical } \\
\text { (Incubation) }\end{array}$ & $\begin{array}{l}187.83 \pm 6.76 \mathrm{~nm} \\
(\mathrm{DLS}) / \\
-9.57 \pm 0.38 \mathrm{mV}\end{array}$ & No & Yes & I.V. & 2018 & [100] \\
\hline Colorectal cancer & Mouse & HEK293T & siSurvivin & $\begin{array}{l}\text { Folate, PSMA RNA } \\
\text { aptamer \& EGFR RNA } \\
\text { aptamer (All } \\
\text { conjugated to 3WJ) }\end{array}$ & $\begin{array}{l}\text { After isolation EVs, } \\
\text { Chemical (ExoFect } \\
\text { Exosome } \\
\text { transfection kit) }\end{array}$ & $\begin{array}{l}103-120 \mathrm{~nm}(\mathrm{NTA}) \\
\mid-15.6 \pm 27.9 \mathrm{mV}\end{array}$ & Yes & Yes & I.V. & 2018 & [80] \\
\hline Colorectal cancer & Mouse & $\begin{array}{l}\text { THLG-293 T } \\
\text { \& LG-293 T }\end{array}$ & $\begin{array}{l}\text { 5-Fluorouracil (5- } \\
\text { FU) \& Anti-miR-21 }\end{array}$ & Her2 binding affibody & $\begin{array}{l}\text { After isolation EVs, } \\
\text { Physical } \\
\text { (Electroporation) }\end{array}$ & $\begin{array}{l}110 \pm 11.3 \mathrm{~nm} \\
(\mathrm{DLS}) / \\
-11 \pm 2.7 \mathrm{mV}\end{array}$ & No & Yes & I.V. & 2020 & [101] \\
\hline Colorectal cancer & Mouse & HEK-293 T & SIRP $\alpha$ protein & SIRP $\propto$ protein & $\begin{array}{l}\text { Before EVs isolation, } \\
\text { Transfection based } \\
\text { (Plasmid) }\end{array}$ & $\begin{array}{l}\text { About } 100 \mathrm{~nm} \\
\text { (DLS) }\end{array}$ & No & No & Local & 2018 & [102] \\
\hline Colorectal cancer & Mouse & НЕК293Т & SIRP $\alpha$ proteins & SIRP $\alpha$ proteins & $\begin{array}{l}\text { Before EVs isolation, } \\
\text { Transfection based } \\
\text { (Plasmid) }\end{array}$ & $\begin{array}{l}\text { About } 100 \mathrm{~nm} \\
\text { (DLS) }\end{array}$ & No & Yes & I.V. \& Local & 2017 & [103] \\
\hline Colorectal cancer & Mouse & $\begin{array}{l}\text { CT26-CIITA } \\
\text { cells }\end{array}$ & $\begin{array}{l}\text { MHC class II } \\
\text { molecule }\end{array}$ & MHC class II molecule & $\begin{array}{l}\text { Before EVs isolation, } \\
\text { Transfection based } \\
\text { (Retrovirus) }\end{array}$ & NA & No & No & I.D. & 2013 & [104] \\
\hline Colorectal cancer & Mouse & $\begin{array}{l}\text { LL/2, MC-38, } \\
\text { A549 \& } \\
\text { human liver } \\
\text { samples }\end{array}$ & $\begin{array}{l}\text { Oncolytic } \\
\text { adenovirus Ad5/3- } \\
\text { CD40L }\end{array}$ & None & $\begin{array}{l}\text { Before EVs isolation, } \\
\text { Transfection based } \\
\text { (Adenovirus) }\end{array}$ & $\begin{array}{l}50-400 \mathrm{~nm}(\mathrm{NTA}) / \\
\text { About }-40 \mathrm{mV}\end{array}$ & No & Yes & I.V. & 2019 & [105] \\
\hline \multicolumn{12}{|l|}{ Cervical cancer } \\
\hline Cervical cancer & Mouse & HeLa & Paclitaxel & None & $\begin{array}{l}\text { Before EVs isolation, } \\
\text { Ultraviolet } \\
\text { irradiation }\end{array}$ & $\begin{array}{l}285.58 \pm 2.95 \mathrm{~nm}, \\
\text { PDI } 0.104 \pm 0.106 \\
\text { (DLS) }\end{array}$ & No & No & I.V. & 2020 & [106] \\
\hline Cervical cancer & Mouse & $\begin{array}{l}\text { THP-1 } \\
\text { macrophages }\end{array}$ & Doxorubicin & $\begin{array}{l}\text { RGD, sulfhydryl } \\
\text { groups, AuNRs \& Folic } \\
\text { acid }\end{array}$ & $\begin{array}{l}\text { After isolation EVs, } \\
\text { Physical } \\
\text { (Electroporation) }\end{array}$ & $\begin{array}{l}\text { 30-300 nm } \\
\left(\text { empty }^{\#}, \text { DLS) }\right.\end{array}$ & No & Yes & I.V. & 2018 & [107] \\
\hline Cervical cancer & Rat & Bovine milk & Curcumin & None & $\begin{array}{l}\text { After isolation EVs, } \\
\text { Chemical (Mixing } \\
\text { with ethanol: } \\
\text { acetonitrile) }\end{array}$ & $\begin{array}{l}93 \pm 6 \mathrm{~nm}, \text { PDI } \\
0.21 \pm 0.04 \text { (DLS) }\end{array}$ & No & No & Oral & 2017 & [108] \\
\hline Cervical cancer & Mouse & Macrophages & Doxorubicin & $\begin{array}{l}\text { Biotin, streptavidin- } \\
\text { modified iron oxide } \\
\text { nanoparticlesSA- } \\
\text { IONPs \& Folic acid }\end{array}$ & $\begin{array}{l}\text { After isolation EVs, } \\
\text { Physical } \\
\text { (Electroporation) }\end{array}$ & $\begin{array}{l}100-1000 \mathrm{~nm} \\
\text { (DLS) / About } \\
-10 \mathrm{mV}\end{array}$ & No & Yes & I.V. & 2017 & [109] \\
\hline Cervical cancer & Mouse & $\begin{array}{l}\text { THP-1 } \\
\text { macrophages }\end{array}$ & $\begin{array}{l}\text { m-THPC } \\
\text { photosensitizer }\end{array}$ & None & $\begin{array}{l}\text { Before EVs isolation, } \\
\text { Incubation }\end{array}$ & $550 \pm 50 \mathrm{~nm}(\mathrm{DLS})$ & No & Yes & Local & 2013 & [110] \\
\hline \multicolumn{12}{|c|}{ Digestive system cancer } \\
\hline $\begin{array}{l}\text { Digestive system } \\
\text { cancer }\end{array}$ & Mouse & Bovine milk & siBcl-2 & None & $\begin{array}{l}\text { After isolation EVs, } \\
\text { Physical } \\
\text { (Ultrasound) }\end{array}$ & $68.06 \mathrm{~nm}$ (DLS) & No & No & I.V. & 2020 & [111] \\
\hline Gastric cancer & Mouse & Urinary & $\begin{array}{l}\text { PMA/Au-BSA@Ce6 } \\
\text { nanoparticles }\end{array}$ & None & $\begin{array}{l}\text { After isolation EVs, } \\
\text { Physical } \\
\text { (Electroporation) }\end{array}$ & $\begin{array}{l}75 \pm 7.6 \mathrm{~nm} \\
(\mathrm{DLS} \& \mathrm{NTA}) / \\
-31.4 \pm 3.1 \mathrm{mV}\end{array}$ & Yes & Yes & I.V. & 2020 & [112] \\
\hline
\end{tabular}




\begin{tabular}{|c|c|c|c|c|c|c|c|c|c|c|c|}
\hline Disease/condition & $\begin{array}{l}\text { Animal } \\
\text { model }\end{array}$ & $\begin{array}{l}\text { Donor cell } \\
\text { type }\end{array}$ & $\begin{array}{l}\text { Active } \\
\text { pharmaceutical } \\
\text { ingredient (API) }\end{array}$ & Surface modifications & $\begin{array}{l}\text { API loading } \\
\text { procedure }\end{array}$ & Size/charge ${ }^{*}$ & $\begin{array}{l}\text { Purity } \\
\text { estimation }\end{array}$ & $\begin{array}{l}\text { Biodistribution } \\
\text { studies }\end{array}$ & $\begin{array}{l}\text { Route of } \\
\text { administration }\end{array}$ & $\begin{array}{l}\text { Year of } \\
\text { publication }\end{array}$ & Ref. \\
\hline Gastric cancer & Mouse & HEK293T & Anti-miR-214 & None & $\begin{array}{l}\text { Before EVs isolation, } \\
\text { Transfection based } \\
\text { (Anti-miR) }\end{array}$ & NA & No & No & I.V. & 2018 & [113] \\
\hline Gastric cancer & Mouse & HEK293T & miRNA-29a/c & None & $\begin{array}{l}\text { Before EVs isolation, } \\
\text { Transfection based } \\
\text { (miRNA) }\end{array}$ & NA & No & No & I.V. & 2016 & [114] \\
\hline \multicolumn{12}{|l|}{ Lung cancer } \\
\hline Lewis lung carcinoma & Mouse & $\begin{array}{l}\text { M1 } \\
\text { macrophages }\end{array}$ & Cisplatin & None & $\begin{array}{l}\text { After isolation EVs, } \\
\text { Physical } \\
\text { (Electroporation) }\end{array}$ & $100-300 \mathrm{~nm}(\mathrm{NTA})$ & No & No & I.V. & 2020 & [115] \\
\hline Lewis lung carcinoma & Mouse & $\begin{array}{l}\text { LL/2, MC-38, } \\
\text { A549 \& } \\
\text { human liver } \\
\text { samples }\end{array}$ & $\begin{array}{l}\text { Oncolytic } \\
\text { adenovirus Ad5/3- } \\
\text { CD40L }\end{array}$ & None & $\begin{array}{l}\text { Before EVs isolation, } \\
\text { Transfection based } \\
\text { (Adenovirus) }\end{array}$ & $\begin{array}{l}50-400 \mathrm{~nm}(\mathrm{NTA}) / \\
\text { About }-40 \mathrm{mV}\end{array}$ & No & Yes & I.V. & 2019 & [105] \\
\hline Lewis Lung carcinoma & Mouse & LL/2 cells & $\begin{array}{l}\text { Immunogenic } \\
\text { oncolytic adenovirus } \\
\text { Ad5D24-CpG\& } \\
\text { Paclitaxel }\end{array}$ & None & $\begin{array}{l}\text { After isolation EVs, } \\
\text { Physical } \\
\text { (Incubation); Before } \\
\text { EVs isolation, } \\
\text { Transfection based } \\
\text { (Adenovirus) }\end{array}$ & $\begin{array}{l}50-400 \mathrm{~nm}(\mathrm{NTA}) / \\
\text { About }-40 \mathrm{mV}\end{array}$ & No & Yes & I.V. & 2019 & [116] \\
\hline Lewis lung carcinoma & Mouse & A549 cells & $\begin{array}{l}\text { Cisplatin \& } \\
\text { Methotrexate \& } \\
\text { Doxorubicin \& } \\
\text { Paclitaxel }\end{array}$ & None & $\begin{array}{l}\text { Before EVs isolation, } \\
\text { Ultraviolet } \\
\text { irradiation }\end{array}$ & $\begin{array}{l}300-800 \mathrm{~nm} \\
\text { (empty", DLS) }\end{array}$ & No & Yes & I.P. \& I.V. & 2016 & [117] \\
\hline Lung metastatic cancer & Mouse & $\begin{array}{l}\text { H22 \& } \\
\text { Bel7402 cells }\end{array}$ & Doxorubicin & None & $\begin{array}{l}\text { Before EVs isolation, } \\
\text { Transfection based } \\
\text { (Plasmid) }\end{array}$ & $\begin{array}{l}260 \pm 15 \mathrm{~nm}, \text { PDI } \\
0.145 \pm 0.032 \\
(\mathrm{DLS}) /- \\
11.0 \pm 0.4 \mathrm{mV}\end{array}$ & No & Yes & I.V. & 2019 & [78] \\
\hline $\begin{array}{r}\text { Lung cancer with } \\
\text { mutated KRAS }\end{array}$ & Mouse & Bovine milk & siKRAS & Folic acid & $\begin{array}{l}\text { After isolation EVs, } \\
\text { Physical } \\
\text { (Electroporation); } \\
\text { After isolation EVs, } \\
\text { Chemical (ExoFect } \\
\text { exosomes } \\
\text { transfection kit) }\end{array}$ & NA & No & Yes & I.V. & 2019 & [118] \\
\hline $\begin{array}{l}\text { Lung cancer patients } \\
\text { with malignant } \\
\text { pleural effusion }\end{array}$ & Mouse & $\begin{array}{l}\text { LLC, MC38, } \\
\text { B16-F10, } \\
\text { A549 \& MCF- } \\
7\end{array}$ & Methotrexate & None & $\begin{array}{l}\text { Before EVs isolation, } \\
\text { Ultraviolet } \\
\text { irradiation }\end{array}$ & $\begin{array}{l}30-930 \mathrm{~nm} \text {, mean } \\
\text { at } 264 \mathrm{~nm}(\mathrm{NTA})\end{array}$ & No & Yes & Local & 2019 & [119] \\
\hline Lung cancer & Mouse & LL/2 cells & $\begin{array}{l}\text { Oncolytic } \\
\text { adenovirus Ad5D24 }\end{array}$ & None & $\begin{array}{l}\text { Before isolation EVs, } \\
\text { Transfection based } \\
\text { (Adenovirus) }\end{array}$ & NA & No & Yes & I.V. & 2018 & [120] \\
\hline Lung cancer & Mouse & $\begin{array}{l}\text { Malignant } \\
\text { cells }\end{array}$ & CRISPR/Cas9 & TNF- $\alpha$ & $\begin{array}{l}\text { After isolation EVs, } \\
\text { Physical } \\
\text { (Electroporation) }\end{array}$ & NA & No & No & I.V. & 2019 & [61] \\
\hline Lung cancer & Mouse & A549 cells & $\begin{array}{l}\text { Oncolytic Ad5D24- } \\
\text { CpG \& Paclitaxel }\end{array}$ & None & $\begin{array}{l}\text { Before EVs isolation, } \\
\text { Transfection based } \\
\text { (Adenovirus); After } \\
\text { isolation EVs, } \\
\text { Physical } \\
\text { (Incubation) }\end{array}$ & $\begin{array}{l}50-1000 \mathrm{~nm} \text {, with } \\
\text { major peak around } \\
100 \mathrm{~nm}(\mathrm{NTA}) / \\
\text { About }-40 \mathrm{mV}\end{array}$ & No & Yes & I.V. & 2018 & [121] \\
\hline Lung cancer & Mouse & Bovine milk & Paclitaxel & None & $\begin{array}{l}\text { After isolation EVs, } \\
\text { Chemical (Mixing } \\
\text { with acetonitrile: } \\
\text { ethanol) }\end{array}$ & $\begin{array}{l}108.1 \pm 1.5, \text { PDI } \\
0.190 \pm 0.006 \text { (DLS) } \\
/-7.4 \pm 0.7 \mathrm{mV}\end{array}$ & No & No & Oral & 2017 & [122] \\
\hline
\end{tabular}




\begin{tabular}{|c|c|c|c|c|c|c|c|c|c|c|c|}
\hline Disease/condition & $\begin{array}{l}\text { Animal } \\
\text { model }\end{array}$ & $\begin{array}{l}\text { Donor cell } \\
\text { type }\end{array}$ & $\begin{array}{l}\text { Active } \\
\text { pharmaceutical } \\
\text { ingredient (API) }\end{array}$ & Surface modifications & $\begin{array}{l}\text { API loading } \\
\text { procedure }\end{array}$ & Size/charge ${ }^{*}$ & $\begin{array}{l}\text { Purity } \\
\text { estimation }\end{array}$ & $\begin{array}{l}\text { Biodistribution } \\
\text { studies }\end{array}$ & $\begin{array}{l}\text { Route of } \\
\text { administration }\end{array}$ & $\begin{array}{l}\text { Year of } \\
\text { publication }\end{array}$ & Ref. \\
\hline Lung cancer & Mouse & Bovine milk & $\begin{array}{l}\text { Berry } \\
\text { anthocyanidins }\end{array}$ & None & $\begin{array}{l}\text { After isolation EVs, } \\
\text { Chemical (Mixture } \\
\text { of acetonitrile: } \\
\text { ethanol) }\end{array}$ & $\begin{array}{l}83 \pm 1.7 \mathrm{~nm}, \mathrm{PDI} \\
0.23 \text { (DLS) }\end{array}$ & No & No & Oral & 2017 & [123] \\
\hline Lung cancer & Mouse & Bovine milk & $\begin{array}{l}\text { Paclitaxel \& } \\
\text { Docetaxel \& } \\
\text { Curcumin \& }\end{array}$ & Folic acid & $\begin{array}{l}\text { After isolation EVs, } \\
\text { Chemical (Mixture } \\
\text { with ethanol \& } \\
\text { acetonitrile) }\end{array}$ & $\begin{array}{l}40-100 \mathrm{~nm}(\mathrm{NTA}) \\
\text { PDI } 0.22 \pm 0.06 \\
\text { (DLS) }\end{array}$ & No & Yes & I.V. \& I.P. & 2016 & [124] \\
\hline Lung cancer & Mouse & Bovine milk & Celastrol (CEL) & None & $\begin{array}{l}\text { After isolation EVs, } \\
\text { Chemical (Dissolved } \\
\text { in ethanol) }\end{array}$ & $\begin{array}{l}106 \pm 9 \mathrm{~nm}, \text { PDI } \\
0.15 \text { (DLS) }\end{array}$ & No & No & Oral & 2016 & [125] \\
\hline Lung Cancer & Mouse & $\begin{array}{l}\text { RAW264.7 } \\
\text { Macrophages }\end{array}$ & Paclitaxel & None & $\begin{array}{l}\text { After isolation EVs, } \\
\text { Physical } \\
\text { (Sonication, } \\
\text { electroporation \& } \\
\text { incubation) }\end{array}$ & $\begin{array}{l}\text { 159-217.9 nm } \\
\text { (NTA\&DLS) / } \\
-14.07 \text { to } \\
-9.33 \mathrm{mV}\end{array}$ & No & No & I.N. & 2016 & [126] \\
\hline $\begin{array}{l}\text { Non-small cell lung } \\
\text { cancer }\end{array}$ & Mouse & $\begin{array}{l}\text { MDA-MB- } \\
231 \text { cells }\end{array}$ & miRNA-126 & None & $\begin{array}{l}\text { After isolation EVs, } \\
\text { Chemical } \\
\left(\text { ExoFectin }{ }^{\circledR} \text { kit }\right. \\
\text { transfection) }\end{array}$ & 30-120 nm (DLS) & No & Yes & I.V. & 2020 & [127] \\
\hline $\begin{array}{l}\text { Non-small cell lung } \\
\text { cancer }\end{array}$ & Mouse & $\begin{array}{l}\text { Human } \\
\text { plasma }\end{array}$ & Imperialine & $\begin{array}{l}\text { Integrin } \alpha 3 \beta 1 \text {-binding } \\
\text { octapeptide } \\
\text { cNGQGEQc }\end{array}$ & $\begin{array}{l}\text { After isolation EVs, } \\
\text { Physical (Incubation } \\
\text { \& ultrasonic) }\end{array}$ & $\begin{array}{l}169.9 \pm 50.7 \mathrm{~nm} \\
(\mathrm{NTA}), \text { PDI } 0.192 / \\
-17.3 \pm 4.32 \mathrm{mV}\end{array}$ & Yes & Yes & I.V. & 2019 & [128] \\
\hline $\begin{array}{l}\text { Non-small cell lung } \\
\text { cancer }\end{array}$ & Mouse & $\begin{array}{l}\text { RAW264.7 } \\
\text { Macrophage }\end{array}$ & Paclitaxel & $\begin{array}{l}\text { Aminoethylanisamide- } \\
\text { PEG) }\end{array}$ & $\begin{array}{l}\text { After isolation EVs, } \\
\text { Physical (Sonication } \\
\text { \& incubation) }\end{array}$ & $\begin{array}{l}280.8 \pm 3.1 \\
(\text { NTA\&DLS) / } \\
-4.4 \pm 0.1 \mathrm{mV}\end{array}$ & No & No & I.V. & 2018 & [129] \\
\hline Small cell lung cancer & Mouse & HEK293 & $\begin{array}{l}\text { Soluble fms-like } \\
\text { tyrosine kinase-1 }\end{array}$ & None & $\begin{array}{l}\text { Before EVs isolation, } \\
\text { Transfection based } \\
\text { (Lentivirus) }\end{array}$ & $\begin{array}{l}\text { About } 100 \mathrm{~nm} \\
\text { (NTA) }\end{array}$ & No & No & Local & 2019 & [130] \\
\hline \multicolumn{12}{|l|}{ Liver cancer } \\
\hline $\begin{array}{l}\text { Hepatocellular } \\
\text { carcinoma }\end{array}$ & Mouse & MSCs & miRNA-199a & None & $\begin{array}{l}\text { Before EVs isolation, } \\
\text { Transfection based } \\
\text { (Lentivirus) }\end{array}$ & $80 \pm 1.9 \mathrm{~nm}(\mathrm{NTA})$ & No & No & I.V. & 2020 & [131] \\
\hline $\begin{array}{l}\text { Hepatocellular } \\
\text { carcinoma }\end{array}$ & Mouse & $\begin{array}{l}\mathrm{H} 22 \text { tumor } \\
\text { cell }\end{array}$ & $\begin{array}{l}\mathrm{Bi}_{2} \mathrm{Se}_{3} \text { nanodots \& } \\
\text { doxorubicin }\end{array}$ & None & $\begin{array}{l}\text { Before EVs isolation, } \\
\text { Electroporation \& } \\
\text { ultraviolet } \\
\text { irradiation }\end{array}$ & $\begin{array}{l}366.71 \pm 20.41 \mathrm{~nm} \\
(\mathrm{DLS}) / \\
-12.02 \pm 0.59 \mathrm{mV}\end{array}$ & No & Yes & I.V. & 2020 & [132] \\
\hline $\begin{array}{l}\text { Hepatocellular } \\
\text { carcinoma }\end{array}$ & Mouse & $\begin{array}{l}\mathrm{H} 22 \text { \& } \\
\text { Bel7402 cells }\end{array}$ & Doxorubicin & None & $\begin{array}{l}\text { Before EVs isolation, } \\
\text { Transfection based } \\
\text { (Plasmid) }\end{array}$ & $\begin{array}{l}260 \pm 15 \mathrm{~nm}, \text { PDI } \\
0.145 \pm 0.032 \\
(\mathrm{DLS}) / \\
-11.0 \pm 0.4 \mathrm{mV}\end{array}$ & No & Yes & I.V. & 2019 & [78] \\
\hline $\begin{array}{l}\text { Hepatocellular } \\
\text { carcinoma }\end{array}$ & Mouse & Blood & Doxorubicin & $\begin{array}{l}\text { Superparamagnetic } \\
\text { magnetite colloidal } \\
\text { nanocrystal clusters }\end{array}$ & $\begin{array}{l}\text { After isolation EVs, } \\
\text { Physical } \\
\text { (Incubation) }\end{array}$ & $\begin{array}{l}\text { Peak around } \\
100 \mathrm{~nm} \text { (DLS) }\end{array}$ & No & Yes & I.V. & 2016 & [133] \\
\hline $\begin{array}{l}\text { Hepatocellular } \\
\text { carcinoma }\end{array}$ & Mouse & H22 \& A2780 & Methotrexate & None & $\begin{array}{l}\text { Before EVs isolation, } \\
\text { Ultraviolet } \\
\text { irradiation }\end{array}$ & $\begin{array}{l}100-1000 \mathrm{~nm} \\
(\mathrm{TEM})\end{array}$ & No & No & I.P. & 2012 & [134] \\
\hline $\begin{array}{l}\text { Hepatocellular } \\
\text { carcinoma ascites }\end{array}$ & Mouse & $\begin{array}{l}\text { BM dendritic } \\
\text { cells }\end{array}$ & Doxorubicin & $\begin{array}{l}\text { Tumor derived } \\
\text { antigens }\end{array}$ & $\begin{array}{l}\text { Before EVs isolation, } \\
\text { Ultraviolet } \\
\text { irradiation }\end{array}$ & $\begin{array}{l}\text { About } 400 \mathrm{~nm} \\
\text { (empty }{ }^{\#}, \mathrm{DLS} \text { ) }\end{array}$ & No & Yes & I.V. & 2017 & [135] \\
\hline $\begin{array}{l}\text { Hepatocellular } \\
\text { carcinoma ascites }\end{array}$ & Mouse & A549 cells & $\begin{array}{l}\text { Cisplatin \& } \\
\text { Methotrexate \& } \\
\text { Doxorubicin \& } \\
\text { Paclitaxel }\end{array}$ & None & $\begin{array}{l}\text { Before EVs isolation, } \\
\text { Ultraviolet } \\
\text { irradiation }\end{array}$ & $\begin{array}{l}300-800 \mathrm{~nm} \\
\left.\text { (empty }{ }^{\#}, \mathrm{DLS}\right)\end{array}$ & No & Yes & I.P. \& I.V. & 2016 & [117] \\
\hline
\end{tabular}




\begin{tabular}{|c|c|c|c|c|c|c|c|c|c|c|c|}
\hline Disease/condition & $\begin{array}{l}\text { Animal } \\
\text { model }\end{array}$ & $\begin{array}{l}\text { Donor cell } \\
\text { type }\end{array}$ & $\begin{array}{l}\text { Active } \\
\text { pharmaceutical } \\
\text { ingredient (API) }\end{array}$ & Surface modifications & $\begin{array}{l}\text { API loading } \\
\text { procedure }\end{array}$ & Size/charge* & $\begin{array}{l}\text { Purity } \\
\text { estimation }\end{array}$ & $\begin{array}{l}\text { Biodistribution } \\
\text { studies }\end{array}$ & $\begin{array}{l}\text { Route of } \\
\text { administration }\end{array}$ & $\begin{array}{l}\text { Year of } \\
\text { publication }\end{array}$ & Ref. \\
\hline \multicolumn{12}{|l|}{ Lymphoma } \\
\hline Lymphoma & Mouse & K562 cells & TRAIL protein & TRAIL protein & $\begin{array}{l}\text { Before EVs isolation, } \\
\text { Transfection based } \\
\text { (Lentivirus) }\end{array}$ & 140 nm (NTA) & No & Yes & I.V. \& Local & 2016 & [136] \\
\hline T-cell lymphoma & Mouse & EL4 cells & Doxorubicin & None & $\begin{array}{l}\text { After isolation EVs, } \\
\text { Physical } \\
\text { (Incubation) }\end{array}$ & $37 \mathrm{~nm}$ (DLS) & No & Yes & I.V. & 2018 & [137] \\
\hline \multicolumn{12}{|l|}{ Melanoma } \\
\hline Melanoma & Mouse & MSCs & TNF- $\alpha$ & $\begin{array}{l}\text { Superparamagnetic } \\
\text { iron oxide } \\
\text { nanoparticles (SPION) }\end{array}$ & $\begin{array}{l}\text { Before EVs isolation, } \\
\text { Transfection based } \\
\text { (Plasmid) }\end{array}$ & $40-80 \mathrm{~nm}$ (DLS) & No & Yes & I.V. & 2020 & [138] \\
\hline Melanoma & Mouse & HEK293T & PH20 hyaluronidase & PH20 hyaluronidase & $\begin{array}{l}\text { Before EVs isolation, } \\
\text { Transfection based } \\
\text { (Plasmid) }\end{array}$ & $\begin{array}{l}\text { About } 100 \mathrm{~nm} \\
\text { (DLS) }\end{array}$ & No & No & Local & 2019 & [95] \\
\hline Melanoma & Mouse & MSCs & TRAIL protein & TRAIL protein & $\begin{array}{l}\text { Before EVs isolation, } \\
\text { Transfection based } \\
\text { (Plasmid) }\end{array}$ & $\begin{array}{l}71.9 \text { nm (empty\#, } \\
\text { DLS) }\end{array}$ & No & Yes & I.V. & 2018 & [139] \\
\hline Melanoma & Mouse & B16BL6 cells & $\begin{array}{l}\text { Immunostimulatory } \\
\text { CpG DNA }\end{array}$ & $\begin{array}{l}\text { Streptavidin- } \\
\text { lactadherin }\end{array}$ & $\begin{array}{l}\text { After isolation EVs, } \\
\text { Physical } \\
\text { (Incubation) }\end{array}$ & $\begin{array}{l}109 \pm 10 \mathrm{~nm} \\
\text { (qNano-TRPS) / - } \\
32 \pm 1.6 \mathrm{mV}\end{array}$ & No & No & I.D. & 2016 & [140] \\
\hline Melanoma & Mouse & $\begin{array}{l}\text { Human } \\
\text { umbilical } \\
\text { vein } \\
\text { endothelial } \\
\text { cell }\end{array}$ & siVEGF & $\begin{array}{l}\text { Streptavidin- } \\
\text { conjugated quantum } \\
\text { dots }\end{array}$ & $\begin{array}{l}\text { After isolation EVs, } \\
\text { Physical } \\
\text { (Electroporation) }\end{array}$ & $\begin{array}{l}629.2 \mathrm{~nm}, \mathrm{PDI} \\
0.296 \text { (empty }^{\#}, \\
\text { DLS) }\end{array}$ & No & Yes & Local & 2015 & [141] \\
\hline \multicolumn{12}{|l|}{ Ovarian cancer } \\
\hline Ovarian cancer & Mouse & SKOV3 cells & Triptolide & None & $\begin{array}{l}\text { After isolation EVs, } \\
\text { Physical } \\
\text { (Sonication) }\end{array}$ & $145 \mathrm{~nm}$ (NTA) & No & Yes & I.P. & 2019 & [142] \\
\hline Ovarian cancer & Mouse & $\begin{array}{l}\text { HEK293 \& } \\
\text { SKOV3 cells }\end{array}$ & $\begin{array}{l}\text { CRISPR/Cas9-NLS \& } \\
\text { PARP-1 sgRNA }\end{array}$ & None & $\begin{array}{l}\text { After isolation EVs, } \\
\text { Physical } \\
\text { (Electroporation) }\end{array}$ & $\begin{array}{l}\text { 50-150 nm } \\
\text { (empty", DLS) }\end{array}$ & No & Yes & I.V. \& Local & 2017 & [143] \\
\hline Ovarian cancer & Mouse & Bovine milk & $\begin{array}{l}\text { Berry } \\
\text { anthocyanidins \& } \\
\text { Paclitaxel }\end{array}$ & None & $\begin{array}{l}\text { After isolation EVs, } \\
\text { Chemical (Mixing \& } \\
\text { dissolving with } \\
\text { ethanol) }\end{array}$ & $\begin{array}{l}\text { Not reported (NTA } \\
\text { and DLS) }\end{array}$ & No & No & Oral & 2017 & [144] \\
\hline Ovarian cancer & Mouse & $\begin{array}{l}\text { MDA-MB- } \\
231 \text { \& STOSE }\end{array}$ & Doxorubicin & None & $\begin{array}{l}\text { After isolation EVs, } \\
\text { Physical } \\
\text { (Electroporation) }\end{array}$ & $101 \mathrm{~nm}(\mathrm{NTA})$ & No & No & I.P. & 2016 & [86] \\
\hline Ovarian cancer & Mouse & H22 \& A2780 & Methotrexate & None & $\begin{array}{l}\text { Before EVs isolation, } \\
\text { Ultraviolet } \\
\text { irradiation }\end{array}$ & $\begin{array}{l}100-1000 \mathrm{~nm} \\
(\text { TEM) }\end{array}$ & No & No & I.P. & 2012 & [134] \\
\hline \multicolumn{12}{|l|}{ Pancreatic cancer } \\
\hline Pancreatic cancer & Mouse & PANC-1 & SIPAK4 & None & $\begin{array}{l}\text { After isolation EVs, } \\
\text { Physical } \\
\text { (Electroporation) }\end{array}$ & $\begin{array}{l}97.1 \pm 1.7 \mathrm{~nm} \\
(\text { empty\#, NTA) / } \\
-15 \text { to }-10 \mathrm{mV}\end{array}$ & Yes & No & Local & 2021 & [145] \\
\hline Pancreatic cancer & Mouse & PANC-1 & Gemcitabine & None & $\begin{array}{l}\text { After isolation EVs, } \\
\text { Physical (Incubation } \\
\text { \& sonication) }\end{array}$ & $\begin{array}{l}\text { About } 100-150 \mathrm{~nm} \\
\text { (NTA) }\end{array}$ & No & Yes & I.V. & 2020 & [146] \\
\hline Pancreatic cancer & Mouse & MSCs & siKRAS ${ }^{G 12 D}$ & None & $\begin{array}{l}\text { After isolation EVs, } \\
\text { Physical } \\
\text { (Electroporation) }\end{array}$ & $\begin{array}{l}179 \mathrm{~nm} \text { (empty } \\
\text { NTA) }\end{array}$ & Yes & Yes & I.P. & 2018 & [147] \\
\hline Pancreatic cancer & Mouse & MSCs & $\begin{array}{l}\text { siKRASG12D \& } \\
\text { pLKO.1- }\end{array}$ & CD47 & $\begin{array}{l}\text { After isolation EVs, } \\
\text { Physical }\end{array}$ & $\begin{array}{l}\text { Peak around } \\
150 \mathrm{~nm}\left(\text { empty }^{\#} \text {, }\right.\end{array}$ & Yes & Yes & I.P. & 2017 & [148] \\
\hline
\end{tabular}




\begin{tabular}{|c|c|c|c|c|c|c|c|c|c|c|c|}
\hline Disease/condition & $\begin{array}{l}\text { Animal } \\
\text { model }\end{array}$ & $\begin{array}{l}\text { Donor cell } \\
\text { type }\end{array}$ & $\begin{array}{l}\text { Active } \\
\text { pharmaceutical } \\
\text { ingredient (API) }\end{array}$ & Surface modifications & $\begin{array}{l}\text { API loading } \\
\text { procedure }\end{array}$ & Size/charge* & $\begin{array}{l}\text { Purity } \\
\text { estimation }\end{array}$ & $\begin{array}{l}\text { Biodistribution } \\
\text { studies }\end{array}$ & $\begin{array}{l}\text { Route of } \\
\text { administration }\end{array}$ & $\begin{array}{l}\text { Year of } \\
\text { publication }\end{array}$ & Ref. \\
\hline $\begin{array}{l}\text { Pancreatic ductal } \\
\text { adenocarcinoma }\end{array}$ & Mouse & hucMSCs & $\begin{array}{l}\text { shKRASG12D } \\
\text { miRNA-145-5p }\end{array}$ & None & $\begin{array}{l}\text { (Electroporation) } \\
\text { After isolation EVs, } \\
\text { Chemical (Exo- } \\
\text { Fect } \\
\text { Transfectione } \\
\text { Reagent) }\end{array}$ & $\begin{array}{l}\text { NTA) } \\
52.5-185.5 \mathrm{~nm} \\
\text { (empty\#, NTA) }\end{array}$ & No & No & Local & 2019 & [149] \\
\hline $\begin{array}{l}\text { Pancreatic ductal } \\
\text { adenocarcinoma }\end{array}$ & Mouse & $\begin{array}{l}\text { K989 cells \& } \\
\text { tumor- } \\
\text { associated } \\
\text { macrophages }\end{array}$ & Anti-miR-365 & None & $\begin{array}{l}\text { Before EVs isolation, } \\
\text { Chemical } \\
\text { Transfection based } \\
\text { (Plasmid) }\end{array}$ & $\begin{array}{l}135 \mathrm{~nm} \text { (empty", } \\
\text { NTA) }\end{array}$ & No & No & I.P. & 2018 & [150] \\
\hline \multicolumn{12}{|l|}{ Prostate cancer } \\
\hline Prostate cancer & Mouse & HEK293T & siSurvivin & $\begin{array}{l}\text { Folate, PSMA RNA } \\
\text { aptamer \& EGFR RNA } \\
\text { aptamer (All } \\
\text { conjugated to 3WJ) }\end{array}$ & $\begin{array}{l}\text { After isolation EVs, } \\
\text { Chemical (ExoFect } \\
\text { Exosome } \\
\text { transfection kit) }\end{array}$ & $\begin{array}{l}103-120 \mathrm{~nm}(\mathrm{NTA}) \\
/-15.6 \pm 27.9 \mathrm{mV}\end{array}$ & Yes & Yes & I.V. & 2018 & [80] \\
\hline Prostate cancer & Mouse & HEK293 & $\begin{array}{l}\text { PH20 hyaluronidase } \\
\text { \& Doxorubicin }\end{array}$ & PH20 hyaluronidase & $\begin{array}{l}\text { Before EVs isolation, } \\
\text { Transfection based } \\
\text { (Plasmid); After } \\
\text { isolation EVs, } \\
\text { Physical } \\
\text { (Incubation) }\end{array}$ & 95 nm (DLS) & No & Yes & Local & 2018 & [83] \\
\hline \multicolumn{12}{|l|}{ Sarcoma } \\
\hline Fibrosarcoma & Mouse & $\begin{array}{l}\text { HT1080 \& } \\
\text { HeLa }\end{array}$ & $\operatorname{Doxil}^{\circledR}$ & None & $\begin{array}{l}\text { After isolation EVs, } \\
\text { Physical (Incubation } \\
\text { followed by } \\
\text { extrusion) }\end{array}$ & $87.7 \pm 2.4(\mathrm{NTA})$ & Yes & Yes & I.V. & 2020 & [151] \\
\hline Sarcoma & Mouse & $\begin{array}{l}\text { Fibroblast } \\
\text { L929 }\end{array}$ & siTGF- $\beta 1$ & None & $\begin{array}{l}\text { Before EVs isolation, } \\
\text { Transfection based } \\
\text { (siRNA) }\end{array}$ & $\begin{array}{l}\text { 60-100 nm } \\
\left.\text { (empty\# }{ }^{\#}, \mathrm{TEM}\right)\end{array}$ & No & Yes & I.V. & 2014 & [152] \\
\hline Sarcoma & Mouse & $\begin{array}{l}\text { THP-1 \& } \\
293 \text { T }\end{array}$ & Anti-miR-150 & None & $\begin{array}{l}\text { Before EVs isolation, } \\
\text { Chemical } \\
\text { Transfection based } \\
\text { (Anti-miR) }\end{array}$ & NA & No & No & I.V. & 2013 & [153] \\
\hline \multicolumn{12}{|l|}{ Other cancer } \\
\hline $\begin{array}{l}\text { Carcinoma (KB } \\
\text { xenograft) }\end{array}$ & Mouse & Ginger root & siSurvivin & Folic acid & $\begin{array}{l}\text { After isolation EVs, } \\
\text { Chemical (ExoFect } \\
\text { Exosome } \\
\text { transfection kit) }\end{array}$ & $\begin{array}{l}\text { About } 100 \mathrm{~nm} \\
\text { (NTA) }\end{array}$ & Yes & No & I.V. & 2018 & [154] \\
\hline $\begin{array}{l}\text { Chronic myelogenous } \\
\text { leukemia }\end{array}$ & Mouse & HEK293T & $\begin{array}{l}\text { Imatinib \& siBCR- } \\
\mathrm{ABL}\end{array}$ & IL3 & $\begin{array}{l}\text { Before EVs isolation, } \\
\text { Transfection based } \\
\text { (siRNA) \& } \\
\text { Incubation }\end{array}$ & $\begin{array}{l}\text { 30-60 (empty } \\
\text { DLS) }\end{array}$ & No & Yes & I.P. & 2017 & [155] \\
\hline Nasopharyngeal cancer & Mouse & HUVECs & $\begin{array}{l}\text { Anti-miR-BART10- } \\
\text { 5p \& Anti-miR-18a }\end{array}$ & iRGD & $\begin{array}{l}\text { Before EVs isolation, } \\
\text { Transfection based } \\
\text { (Plasmid) }\end{array}$ & $\begin{array}{l}\text { About } 100 \mathrm{~nm} \\
\text { (empty", DLS) }\end{array}$ & No & Yes & I.V. & 2020 & [156] \\
\hline Neuroendocrine cancer & Mouse & НЕК293 & $\begin{array}{l}\text { Verrucarin A \& } \\
\text { romidepsin }\end{array}$ & Anti-SSTR2 mAb & $\begin{array}{l}\text { After isolation EVs, } \\
\text { Physical } \\
\text { (Incubation) }\end{array}$ & $\begin{array}{l}125 \pm 6 \mathrm{~nm} \\
\left(\text { empty }^{\#}, \mathrm{NTA}\right)\end{array}$ & No & Yes & I.V. & 2020 & [157] \\
\hline Renal cell carcinoma & Mouse & RCC cells & miRNA-31-5p & None & $\begin{array}{l}\text { Before EVs isolation, } \\
\text { Transfection based } \\
\text { (miRNA mimetics) }\end{array}$ & $\begin{array}{l}53.3-57.8 \mathrm{~nm} \\
\left.\text { (empty }{ }^{\#}, \mathrm{NTA}\right)\end{array}$ & No & No & I.V. & 2020 & [158] \\
\hline Schwannoma & Mouse & HEK-293 T & $\begin{array}{l}\text { CD-UPRT mRNA \& } \\
\text { protein }\end{array}$ & None & $\begin{array}{l}\text { Before EVs isolation, } \\
\text { Transfection based } \\
\text { (Plasmid) }\end{array}$ & $\begin{array}{l}159 \text { nm (empty", } \\
\text { NTA) }\end{array}$ & No & No & Local & 2013 & [159] \\
\hline Thyroid cancer & Mouse & $\begin{array}{l}\text { Malignant } \\
\text { cells }\end{array}$ & CRISPR/Cas9 & TNF- $\alpha$ & $\begin{array}{l}\text { After isolation EVs, } \\
\text { Physical } \\
\text { (Electroporation) }\end{array}$ & NA & No & No & I.V. & 2019 & [61] \\
\hline
\end{tabular}


Preclinical studies of EVs as drug delivery systems for cardiovascular disease treatment.

\begin{tabular}{|c|c|c|c|c|c|c|c|c|c|c|c|}
\hline Disease/condition & $\begin{array}{l}\text { Animal } \\
\text { model }\end{array}$ & EV donor cell type & $\begin{array}{l}\text { Active } \\
\text { pharmaceutical } \\
\text { ingredient (API) }\end{array}$ & $\begin{array}{l}\text { EV surface } \\
\text { modifications }\end{array}$ & API loading procedure & EV size/charge* & $\begin{array}{l}\text { EV } \\
\text { purity } \\
\text { stated }\end{array}$ & $\begin{array}{l}\text { Biodistribution } \\
\text { studies }\end{array}$ & $\begin{array}{l}\text { Route of } \\
\text { administration }\end{array}$ & $\begin{array}{l}\text { Year of } \\
\text { publication }\end{array}$ & Ref. \\
\hline \multicolumn{12}{|l|}{ Myocardial infarction } \\
\hline $\begin{array}{l}\text { Acute myocardial } \\
\text { infarction }\end{array}$ & Rat & MSCs & IncRNA-H19 & None & $\begin{array}{l}\text { Before EV isolation, Stimulated } \\
\text { by atorvastatin }\end{array}$ & $\begin{array}{l}\text { About } 100 \mathrm{~nm} \\
\text { (NTA) }\end{array}$ & No & No & Local & 2019 & [160] \\
\hline $\begin{array}{l}\text { Acute myocardial } \\
\text { infarction }\end{array}$ & Rat & Adipose-stem cells & miRNA-126 & None & $\begin{array}{l}\text { Before EVs isolation, } \\
\text { Transfection based (miRNA) }\end{array}$ & $50-100 \mathrm{~nm}(\mathrm{NTA})$ & No & No & I.V. & 2018 & [161] \\
\hline $\begin{array}{l}\text { Acute myocardial } \\
\text { infarction }\end{array}$ & Rat & MSCs & Akt & None & $\begin{array}{l}\text { Before EVs isolation, } \\
\text { Transfection based } \\
\text { (Adenovirus) }\end{array}$ & $\begin{array}{l}\text { About } 100 \mathrm{~nm} \\
\text { (NTA) }\end{array}$ & No & No & I.V. & 2017 & [162] \\
\hline $\begin{array}{l}\text { Acute Myocardial } \\
\text { infarction }\end{array}$ & Rat & MSCs & TIMP2 protein & None & $\begin{array}{l}\text { Before EVs isolation, } \\
\text { Transfection based (Lentivirus) }\end{array}$ & 40-90 nm (TEM) & No & No & Local & 2019 & [163] \\
\hline $\begin{array}{l}\text { Acute Myocardial } \\
\text { infarction }\end{array}$ & Mouse & HEK293T & miRNA-21 & None & $\begin{array}{l}\text { Before EVs isolation, } \\
\text { Transfection based (Plasmid) }\end{array}$ & 30-150 nm (NTA) & No & No & Local & 2019 & [164] \\
\hline $\begin{array}{l}\text { Acute Myocardial } \\
\text { infarction }\end{array}$ & Mouse & $\begin{array}{l}\text { Human peripheral } \\
\text { blood }\end{array}$ & miRNA-21 & None & $\begin{array}{l}\text { After isolation EVs, Chemical } \\
\text { (Exo-Fect }{ }^{\mathrm{MM}} \text { Exosome } \\
\text { Transfection kit) }\end{array}$ & $\begin{array}{l}\text { About } 104 \mathrm{~nm} \\
\left.\text { (empty }{ }^{\#}, \mathrm{NTA}\right)\end{array}$ & No & No & Local & 2019 & [165] \\
\hline $\begin{array}{l}\text { Acute Myocardial } \\
\text { infarction }\end{array}$ & Mouse & MSCs & $\begin{array}{l}\text { Stromal-derived } \\
\text { factor } 1\end{array}$ & None & $\begin{array}{l}\text { Before EVs isolation, } \\
\text { Transfection based (Plasmid) }\end{array}$ & $\begin{array}{l}112.2 \pm 19.6 \mathrm{~nm} \\
\left(\text { empty }^{\#}, \text { NTA }\right)\end{array}$ & No & No & Local & 2019 & [166] \\
\hline $\begin{array}{l}\text { Myocardial ischemia } \\
\text { reperfusion injury }\end{array}$ & Rat & BMSCs & miRNA 125b & None & $\begin{array}{l}\text { Before EVs isolation, } \\
\text { Transfection based (miRNA) }\end{array}$ & $\begin{array}{l}60-100 \mathrm{~nm} \\
\left(\mathrm{empty}^{\#}, \mathrm{TEM}\right)\end{array}$ & No & No & Local & 2020 & [167] \\
\hline \multicolumn{12}{|l|}{ Stroke } \\
\hline Cerebral ischemia & Rat & MSCs & miRNA-223-3p & None & $\begin{array}{l}\text { Before EVs isolation, } \\
\text { Transfection based (Lentivirus) }\end{array}$ & 30-150 nm (TEM) & No & No & I.V. & 2020 & [168] \\
\hline Cerebral ischemia & Mouse & BMSCs & Curcumin & $\begin{array}{l}\mathrm{c}(\mathrm{RGDyK}) \\
\text { peptide }\end{array}$ & $\begin{array}{l}\text { After isolation EVs, physical } \\
\text { (Incubation) }\end{array}$ & $\begin{array}{l}\text { About } 107 \mathrm{~nm} \\
\text { (NTA) / -21.6 mV } \\
(\text { empty })\end{array}$ & No & Yes & I.V. & 2018 & [169] \\
\hline Cerebral ischemia & Mouse & HEK293T & $\begin{array}{l}\text { Bioactive nerve } \\
\text { growth factor }\end{array}$ & RVG peptide & $\begin{array}{l}\text { Before EVs isolation, } \\
\text { Transfection based (Plasmid) }\end{array}$ & $20-500 \mathrm{~nm}(\mathrm{NTA})$ & No & Yes & I.V. & 2020 & [170] \\
\hline Cerebral ischemia & Mouse & BM-MSCs & miRNA-124 & RVG peptide & $\begin{array}{l}\text { After isolation EVs, Physical } \\
\text { (Electroporation) }\end{array}$ & NA & No & Yes & I.V. & 2017 & [171] \\
\hline $\begin{array}{l}\text { Cerebral ischemia- } \\
\text { reperfusion injury }\end{array}$ & Rat & $\begin{array}{l}\text { RAW264.7 } \\
\text { macrophage }\end{array}$ & Curcumin & None & $\begin{array}{l}\text { Before EVs isolation, } \\
\text { Incubation }\end{array}$ & $\begin{array}{l}110.1 \pm 8.1 \mathrm{~nm} \\
(\mathrm{DLS})\end{array}$ & No & No & I.V. & 2020 & [172] \\
\hline $\begin{array}{l}\text { Cerebral ischemia- } \\
\text { reperfusion injury }\end{array}$ & Rat & $\begin{array}{l}\text { Adipose-derived } \\
\text { stem cells }\end{array}$ & $\begin{array}{l}\text { Pigment } \\
\text { epithelium- } \\
\text { derived factor }\end{array}$ & None & $\begin{array}{l}\text { Before EVs isolation, Chemical } \\
\text { Transfection based (Plasmid) }\end{array}$ & $\begin{array}{l}\text { About } 100 \mathrm{~nm} \\
\text { (TEM) }\end{array}$ & No & No & Local & 2018 & [173] \\
\hline $\begin{array}{l}\text { Cerebral ischemia- } \\
\text { reperfusion injury }\end{array}$ & Mouse & MSCs & Curcumin & None & $\begin{array}{l}\text { After isolation EVs, Physical } \\
\text { (Incubation followed by } \\
\text { freeze-thaw cycle) }\end{array}$ & $\begin{array}{l}\text { About } 118 \mathrm{~nm} \\
\text { (empty\#, NTA) }\end{array}$ & No & No & I.N. & 2016 & [174] \\
\hline \multicolumn{12}{|c|}{ Other cardiovascular disease } \\
\hline $\begin{array}{l}\text { Aging-induced vascular } \\
\text { dysfunction }\end{array}$ & Mouse & UMSCs & miRNA-675 & None & $\begin{array}{l}\text { Before EVs isolation, } \\
\text { Transfection based (miRNA) }\end{array}$ & NA & No & No & Local & 2019 & [175] \\
\hline Atherosclerosis & Mouse & THP-1 \& 293 T & Anti-miR-150 & None & $\begin{array}{l}\text { Before EVs isolation, Chemical } \\
\text { Transfection based (Anti-miR) }\end{array}$ & NA & No & No & I.V. & 2013 & [153] \\
\hline Cardiotoxicity & Mouse & Blood & miRNA-21 & None & $\begin{array}{l}\text { After isolation EVs, Physical } \\
\text { (Electroporation) }\end{array}$ & $\begin{array}{l}40-400 \mathrm{~nm} \\
\left.\text { (empty }{ }^{\#}, \mathrm{NTA}\right)\end{array}$ & No & No & I.V. & 2020 & [176] \\
\hline $\begin{array}{l}\text { Diabetic } \\
\quad \text { cardiomyopathy }\end{array}$ & Mouse & $\begin{array}{l}\text { Hsp20-TG } \\
\text { Cardiomyocytes }\end{array}$ & Hsp20 & None & $\begin{array}{l}\text { Before EVs isolation, } \\
\text { Transfection based } \\
\text { (Adenovirus) }\end{array}$ & $\begin{array}{l}\text { About } 100 \mathrm{~nm} \\
\text { (DLS) }\end{array}$ & No & No & I.V. & 2016 & [177] \\
\hline $\begin{array}{l}\text { Transthyretin } \\
\text { amyloidosis }\end{array}$ & Mouse & $\begin{array}{l}\text { Human primary } \\
\text { neonatal fibroblasts } \\
\text { \& HEK293T }\end{array}$ & $\begin{array}{l}\text { siTTR with pre- } \\
\text { miR- } 45 \text { backbone }\end{array}$ & None & $\begin{array}{l}\text { Before EVs isolation, } \\
\text { Transfection based (Lentivirus) }\end{array}$ & $\begin{array}{l}\text { About } 100 \mathrm{~nm} \\
\text { (NTA) }\end{array}$ & No & Yes & I.V. & 2020 & [178] \\
\hline
\end{tabular}


Table 2c

Preclinical studies of EVs as drug delivery systems for neurological disease treatment.

\begin{tabular}{|c|c|c|c|c|c|c|c|c|c|c|c|}
\hline Disease/condition & $\begin{array}{l}\text { Animal } \\
\text { model }\end{array}$ & $\begin{array}{l}\text { EV donor } \\
\text { cell type }\end{array}$ & $\begin{array}{l}\text { Active } \\
\text { pharmaceutical } \\
\text { ingredient (API) }\end{array}$ & $\begin{array}{l}\text { EV surface } \\
\text { modifications }\end{array}$ & API loading procedure & EV size/charge* & $\begin{array}{l}\text { EV } \\
\text { purity } \\
\text { stated }\end{array}$ & $\begin{array}{l}\text { Biodistribution } \\
\text { studies }\end{array}$ & $\begin{array}{l}\text { Route of } \\
\text { administration }\end{array}$ & $\begin{array}{l}\text { Year of } \\
\text { publication }\end{array}$ & Ref. \\
\hline \multicolumn{12}{|l|}{ Alzheimer's disease } \\
\hline $\begin{array}{l}\text { Alzheimer's } \\
\text { disease }\end{array}$ & Mouse & $\begin{array}{l}\text { RAW264.7 } \\
\text { Macrophages }\end{array}$ & Curcumin & None & Before EVs isolation, Incubation & $\begin{array}{l}117.4 \pm 10.5 \mathrm{~nm}-4.9 \mathrm{mV} \\
\text { (DLS) }\end{array}$ & No & Yes & I.P. & 2019 & [179] \\
\hline $\begin{array}{l}\text { Alzheimer's } \\
\text { disease }\end{array}$ & Mouse & $\begin{array}{l}\text { Dendritic } \\
\text { cells }\end{array}$ & siBACE1 & RVG peptide & $\begin{array}{l}\text { After isolation EVs, Physical } \\
\text { (Electroporation) }\end{array}$ & $\begin{array}{l}\text { About } 88 \mathrm{~nm} \text { (empty", } \\
\text { NTA) }\end{array}$ & No & No & I.V. & 2011 & [180] \\
\hline \multicolumn{12}{|l|}{ Parkinson's disease } \\
\hline $\begin{array}{l}\text { Parkinson's } \\
\text { disease }\end{array}$ & Mouse & Primary DCs & $\begin{array}{l}\text { Anti-alpha- } \\
\text { synuclein } \\
\text { shRNA- } \\
\text { minicircle }\end{array}$ & RVG peptide & $\begin{array}{l}\text { After isolation EVs, Physical } \\
\text { (Electroporation) }\end{array}$ & About $100 \mathrm{~nm}$ (NTA) & No & No & I.V. & 2019 & [181] \\
\hline $\begin{array}{l}\text { Parkinson's } \\
\text { disease }\end{array}$ & Mouse & НЕК293Т & $\begin{array}{l}\text { DNA Aptamer } \\
\text { F5R2 } \alpha \text { - } \\
\text { Synuclein }\end{array}$ & RVG peptide & $\begin{array}{l}\text { After isolation EVs, Physical } \\
\text { (Incubation) }\end{array}$ & $\begin{array}{l}\text { About } 100 \mathrm{~nm} \text { (empty", } \\
\text { TEM) }\end{array}$ & No & No & I.P. & 2019 & [182] \\
\hline $\begin{array}{l}\text { Parkinson's } \\
\text { disease }\end{array}$ & Mouse & HEK293T & Catalase mRNA & $\begin{array}{l}\text { CD63-L7Ae, } \\
\text { RVG peptide \& } \\
\text { Cx43 S368A }\end{array}$ & $\begin{array}{l}\text { Before EVs isolation, Transfection } \\
\text { based (Plasmid) }\end{array}$ & About $100 \mathrm{~nm}$ (NTA) & No & Yes & S.C. & 2018 & [183] \\
\hline $\begin{array}{l}\text { Parkinson's } \\
\text { disease }\end{array}$ & Mouse & Blood & Dopamine & None & $\begin{array}{l}\text { After isolation EVs, Physical } \\
\text { (Incubation) }\end{array}$ & 70-100 nm (TRPS) & Yes & Yes & I.V. & 2018 & [184] \\
\hline $\begin{array}{l}\text { Parkinson's } \\
\text { disease }\end{array}$ & Mouse & $\begin{array}{l}\text { RAW264.7 } \\
\text { Macrophages }\end{array}$ & Catalase & None & $\begin{array}{l}\text { After isolation EVs, Physical } \\
\text { (Sonication, incubation or } \\
\text { extrusion); After isolation EVs, } \\
\text { Chemical (Incubation with } \\
\text { saponin) }\end{array}$ & $\begin{array}{l}100.5-183.7 \text { nm, PDI } 0.2- \\
0.48 \text { (DLS) \& 99.5- } \\
162.4 \mathrm{~nm} \text { (NTA) }\end{array}$ & No & No & I.N. & 2015 & [185] \\
\hline $\begin{array}{l}\text { Parkinson's } \\
\text { disease }\end{array}$ & Mouse & $\begin{array}{l}\text { Murine } \\
\text { dendritic } \\
\text { cells }\end{array}$ & siRNA $\alpha$-Syn & RVG peptide & $\begin{array}{l}\text { After isolation EVs, Physical } \\
\text { (Electroporation) }\end{array}$ & $\begin{array}{l}\text { About } 100 \mathrm{~nm} \text { (empty", } \\
\text { NTA) }\end{array}$ & No & No & I.V. & 2014 & [186] \\
\hline \multicolumn{12}{|c|}{ Other neurological disease } \\
\hline $\begin{array}{l}\text { Huntington's } \\
\text { disease }\end{array}$ & Mouse & U87 cells & hsiHtt & None & $\begin{array}{l}\text { After isolation EVs, Chemical } \\
\text { (Incubation) }\end{array}$ & $\begin{array}{l}\text { About } 140 \mathrm{~nm}(\mathrm{NTA}) /- \\
32 \mathrm{mV}\end{array}$ & No & No & Local & 2016 & [187] \\
\hline Multiple sclerosis & Mouse & MSCs & $\begin{array}{l}\text { LJM-3064 } \\
\text { aptamer }\end{array}$ & $\begin{array}{l}\text { LJM-3064 } \\
\text { aptamer }\end{array}$ & $\begin{array}{l}\text { After isolation EVs, Chemical (Click } \\
\text { chemistry) }\end{array}$ & $\begin{array}{l}133 \pm 15 \mathrm{~nm}(\mathrm{DLS}) / \\
-40.8 \mathrm{mV}\end{array}$ & No & No & I.V. & 2019 & [188] \\
\hline
\end{tabular}


Table 2d

Preclinical studies of EVs as drug delivery systems for inflammatory disease treatment.

\begin{tabular}{|c|c|c|c|c|c|c|c|c|c|c|c|}
\hline Disease/condition & $\begin{array}{l}\text { Animal } \\
\text { model }\end{array}$ & $\begin{array}{l}\text { EV donor cell } \\
\text { type }\end{array}$ & $\begin{array}{l}\text { Active } \\
\text { pharmaceutical } \\
\text { ingredient (API) }\end{array}$ & $\begin{array}{l}\text { EV surface } \\
\text { modifications }\end{array}$ & API loading procedure & EV size/charge* & $\begin{array}{l}\text { EV } \\
\text { purity } \\
\text { stated }\end{array}$ & $\begin{array}{l}\text { Biodistribution } \\
\text { studies }\end{array}$ & $\begin{array}{l}\text { Route of } \\
\text { administration }\end{array}$ & $\begin{array}{l}\text { Year of } \\
\text { publication }\end{array}$ & Ref. \\
\hline \multicolumn{12}{|l|}{ Arthritis } \\
\hline Osteoarthritis & Rat & SMSCs & miRNA-140-5p & None & $\begin{array}{l}\text { Before EVs isolation, } \\
\text { Transfection based } \\
\text { (Lentivirus) }\end{array}$ & $\begin{array}{l}95.01 \pm 35.91 \mathrm{~nm} \\
(\mathrm{DLS})\end{array}$ & No & No & Local & 2017 & [189] \\
\hline Osteoarthritis & Rat & Dendritic cells & miRNA-140 & $\begin{array}{l}\text { Chondrocyte- } \\
\text { affinity } \\
\text { peptide }\end{array}$ & $\begin{array}{l}\text { After isolation EVs, } \\
\text { Physical (Electroporation) }\end{array}$ & $\begin{array}{l}40-200 \mathrm{~nm} \\
\left(\text { empty }^{\#}, \mathrm{NTA}\right)\end{array}$ & No & No & Local & 2020 & [190] \\
\hline Rheumatoid arthritis & Mouse & MSCs & miRNA-150-5p & None & $\begin{array}{l}\text { Before EVs isolation, } \\
\text { Transfection based } \\
\text { (Plasmid) }\end{array}$ & $\begin{array}{l}\text { About } 100 \mathrm{~nm} \\
\text { (DLS) }\end{array}$ & No & No & I.P. & 2018 & [191] \\
\hline \multicolumn{12}{|c|}{ Other inflammatory disease } \\
\hline $\begin{array}{l}\text { Allergic cutaneous } \\
\text { contact dermatitis }\end{array}$ & Mouse & $\mathrm{T}$ cells & miRNA-150 & $\mathrm{Ab} \mathrm{LC}$ & $\begin{array}{l}\text { After isolation EVs, } \\
\text { Physical (Incubation) }\end{array}$ & $\begin{array}{l}\text { About } 130 \mathrm{~nm} \\
\left.\text { (empty }{ }^{\#}, \mathrm{NTA}\right)\end{array}$ & No & No & I.V. & 2013 & [192] \\
\hline $\begin{array}{l}\text { Brain inflammatory } \\
\text { diseases }\end{array}$ & Mouse & $\begin{array}{l}\text { 3T3L1, } 4 \text { T1, CT26, } \\
\text { A20 \& EL4 }\end{array}$ & $\begin{array}{l}\text { Curcumin \& JSI124 } \\
\text { (cucurbitacin I) }\end{array}$ & None & $\begin{array}{l}\text { After isolation EVs, } \\
\text { Physical (Incubation) }\end{array}$ & $\mathrm{NA}$ & No & Yes & I.N. & 2011 & [193] \\
\hline $\begin{array}{l}\text { Central nervous system } \\
\text { injury } \\
\text { neuroinflammation }\end{array}$ & Rat & $\begin{array}{l}\text { Embryonic } \\
\text { cortical neuronal } \\
\text { culture }\end{array}$ & PsiRNA-ASC & None & $\begin{array}{l}\text { After EVs isolation, } \\
\text { Physical (Electroporation) }\end{array}$ & NA & No & No & I.V. & 2016 & [194] \\
\hline Intestinal fibrosis & Rat & BMSCs & miRNA-200b & None & $\begin{array}{l}\text { Before EVs isolation, } \\
\text { Transfection based } \\
\text { (Lentivirus) }\end{array}$ & $\begin{array}{l}\text { About } 500 \mathrm{~nm} \\
\text { (TEM) }\end{array}$ & No & No & I.V. & 2017 & [195] \\
\hline $\begin{array}{l}\text { Inflammation-related } \\
\text { diseases }\end{array}$ & Mouse & $\begin{array}{l}\text { EL4 } \\
\text { \&Macrophages }\end{array}$ & Curcumin & None & $\begin{array}{l}\text { After isolation EVs, } \\
\text { Physical (Incubation) }\end{array}$ & NA & No & Yes & I.P. & 2010 & [196] \\
\hline $\begin{array}{l}\text { Inflammatory bowel } \\
\text { disease }\end{array}$ & Rat & Dendritic cells & IL-10 & IL-10 & $\begin{array}{l}\text { Before EVs isolation, } \\
\text { Incubation }\end{array}$ & NA & No & No & I.P. & 2010 & [197] \\
\hline
\end{tabular}




\begin{tabular}{|c|c|c|c|c|c|c|c|c|c|c|c|}
\hline Disease/condition & $\begin{array}{l}\text { Animal } \\
\text { model }\end{array}$ & $\begin{array}{l}\text { EV donor } \\
\text { cell type }\end{array}$ & $\begin{array}{l}\text { Active } \\
\text { pharmaceutical } \\
\text { ingredient (API) }\end{array}$ & $\begin{array}{l}\text { EV surface } \\
\text { modifications }\end{array}$ & API loading procedure & EV size/charge ${ }^{*}$ & $\begin{array}{l}\text { EV } \\
\text { purity } \\
\text { stated }\end{array}$ & $\begin{array}{l}\text { Biodistribution } \\
\text { studies }\end{array}$ & $\begin{array}{l}\text { Route of } \\
\text { administration }\end{array}$ & $\begin{array}{l}\text { Year of } \\
\text { publication }\end{array}$ & Ref. \\
\hline \multicolumn{12}{|l|}{ Obesity and Diabetes } \\
\hline Diabetes & Mouse & $\begin{array}{l}\text { THP-1 \& } \\
293 \text { T }\end{array}$ & Anti-miR-150 & None & $\begin{array}{l}\text { Before EVs isolation, Chemical } \\
\text { Transfection based (Anti-miR) }\end{array}$ & NA & No & No & I.V. & 2013 & [153] \\
\hline Type-1 diabetes & Mouse & hBMSCs & siFas \& Anti-miR-375 & None & $\begin{array}{l}\text { Before EVs isolation, } \\
\text { Transfection based (Plasmid) }\end{array}$ & $\mathrm{NA}$ & No & Yes & I.V. & 2016 & [198] \\
\hline Obesity & Mouse & HEK293T & $\begin{array}{l}\text { miRNA-148a- } \\
\text { responsive PGC1a } \\
\text { mRNA }\end{array}$ & None & $\begin{array}{l}\text { Before EVs isolation, } \\
\text { Transfection based (miRNA) }\end{array}$ & $50-300 \mathrm{~nm}$ (NTA) & No & Yes & I.V. & 2020 & [199] \\
\hline \multicolumn{12}{|l|}{ Infectious disease } \\
\hline HIV-1 & Mouse & $\begin{array}{l}\text { Dendritic } \\
\text { cells }\end{array}$ & HIV-1-Gp120 & None & $\begin{array}{l}\text { Before EVs isolation, } \\
\text { Transfection based } \\
\text { (Adenovirus) }\end{array}$ & NA & No & No & I.V. & 2012 & [200] \\
\hline Tuberculosis & Mouse & $\begin{array}{l}\text { Bone } \\
\text { marrow } \\
\text { macrophage }\end{array}$ & Mycobacterium RNA & None & $\begin{array}{l}\text { Before EVs isolation, } \\
\text { Transfection based (Bacteria) }\end{array}$ & About $170 \mathrm{~nm}$ (NTA) & No & No & I.T. & 2019 & [201] \\
\hline \multicolumn{12}{|l|}{ Kidney disease } \\
\hline $\begin{array}{l}\text { Acute kidney } \\
\text { injuries }\end{array}$ & Mouse & MSCs & $\begin{array}{l}\text { miRNA-10a-5p \& } \\
\text { miRNA-29a-3p \& } \\
\text { miRNA-127-3p \& } \\
\text { miRNA-486-5p }\end{array}$ & None & $\begin{array}{l}\text { Before EVs isolation, } \\
\text { Transfection based (miRNA) }\end{array}$ & $100-300 \mathrm{~nm}(\mathrm{NTA})$ & No & No & I.V. & 2019 & [202] \\
\hline $\begin{array}{l}\text { Chronic kidney } \\
\text { disease }\end{array}$ & Mouse & $\begin{array}{l}\text { Primary } \\
\text { mouse } \\
\text { satellite } \\
\text { cells }\end{array}$ & miRNA-29 & RVG peptide & $\begin{array}{l}\text { Before EVs isolation, } \\
\text { Transfection based } \\
\text { (Adenovirus) }\end{array}$ & $91 \pm 1.9 \mathrm{~nm}(\mathrm{NTA})$ & No & Yes & Local & 2019 & [203] \\
\hline $\begin{array}{l}\text { Chronic kidney } \\
\text { disease }\end{array}$ & Mouse & BM-MSCs & Erythropoietin & Erythropoietin & Before EVs isolation, Incubation & NA & No & No & I.V. & 2015 & [204] \\
\hline \multicolumn{12}{|l|}{ Liver disease } \\
\hline Acute liver failure & Mouse & hUCMSCs & TNF- $\alpha$ & None & Before EVs isolation, Incubation & 30-150 nm (NTA) & No & No & I.V. & 2020 & [205] \\
\hline Acute liver injury & Mouse & HEK293 & $\begin{array}{l}\text { dCas9- VPR/sgRNA } \\
\text { HGF }\end{array}$ & None & $\begin{array}{l}\text { Before EVs isolation, } \\
\text { Transfection based (Plasmid) }\end{array}$ & $\begin{array}{l}\text { About } 50 \text { and } 1000 \mathrm{~nm} \\
\text { peaks (DLS) / About } \\
140 \mathrm{~nm} \text { (exosome } \\
\text { fraction, NTA) }\end{array}$ & Yes & Yes & I.V. & 2018 & [206] \\
\hline Acute liver injury & Mouse & HEK293T & $\begin{array}{l}\text { miRNA-155 \& C/ebp } \alpha \\
\text { gRNA CRISPR/dCas9 }\end{array}$ & $\begin{array}{l}\text { CD9-HuR } \\
\text { fusion protein }\end{array}$ & $\begin{array}{l}\text { Before EVs isolation, } \\
\text { Transfection based (Lentivirus) }\end{array}$ & 100 nm (empty $\left.{ }^{\#}, \mathrm{DLS}\right)$ & No & Yes & I.V. & 2019 & [207] \\
\hline Hepatitis C & Mouse & Huh7 & shCD81 & None & $\begin{array}{l}\text { Before EVs isolation, } \\
\text { Transfection based (Lentivirus) }\end{array}$ & NA & No & No & I.V. & 2012 & [208] \\
\hline $\begin{array}{l}\text { Muscular disease } \\
\text { Muscular dystrophy }\end{array}$ & Mouse & $\begin{array}{l}\text { Human } \\
\text { 293FT cells }\end{array}$ & CD63-propeptide & $\begin{array}{l}\text { CD63- } \\
\text { propeptide }\end{array}$ & $\begin{array}{l}\text { Before EVs isolation, } \\
\text { Transfection based (Lentivirus) }\end{array}$ & About $110.5 \mathrm{~nm}$ (NTA) & No & Yes & I.V. & 2020 & [209] \\
\hline Muscular dystrophy & Mouse & $\mathrm{C} 2 \mathrm{C} 12$ cells & $\begin{array}{l}\text { Phosphorodiamidate } \\
\text { morpholino oligomer }\end{array}$ & CPO5 peptide & $\begin{array}{l}\text { After isolation EVs, Physical } \\
\text { (Incubation) }\end{array}$ & About $117.1 \mathrm{~nm}$ (NTA) & No & Yes & I.V. & 2018 & [210] \\
\hline \multicolumn{12}{|c|}{ 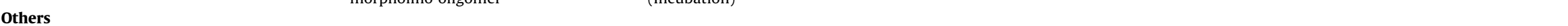 } \\
\hline Cutaneous wound & Rat & MSCs & miRNA-126-3p & None & $\begin{array}{l}\text { Before EVs isolation, } \\
\text { Transfection based (Lentivirus) }\end{array}$ & $64.33 \pm 28.88 \mathrm{~nm}$ (DLS) & No & No & I.V. & 2017 & [211] \\
\hline Immunomodulation & Mouse & $\begin{array}{l}\text { THP1 \& } \\
\text { J774A.1 }\end{array}$ & FasL protein & $\begin{array}{l}\text { AS1411 } \\
\text { aptamer }\end{array}$ & $\begin{array}{l}\text { After isolation EVs, physical } \\
\text { (Incubation) }\end{array}$ & $\begin{array}{l}88.0 \pm 0.09 \text { to } \\
88.9 \pm 1.4 \mathrm{~nm}(\mathrm{DLS}) / \\
-10.0 \pm 1.2 \text { to } \\
-20.4 \pm 2.05 \mathrm{mV}\end{array}$ & No & No & I.P. & 2019 & [212] \\
\hline
\end{tabular}

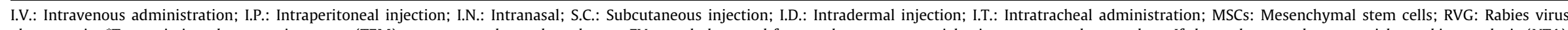

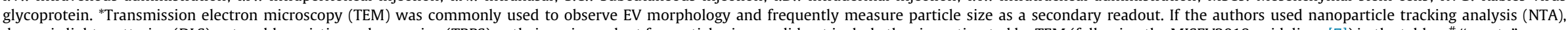

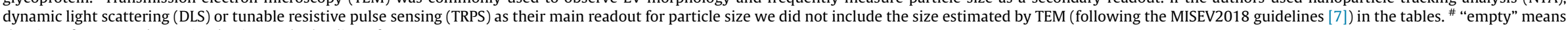
the size of EVs was determined prior to the loading of APIs. 
A

Diseases/conditions

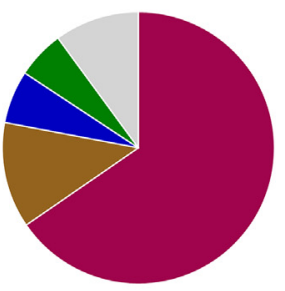

B

Trends in diseases/conditions

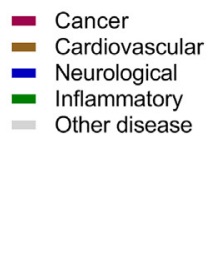

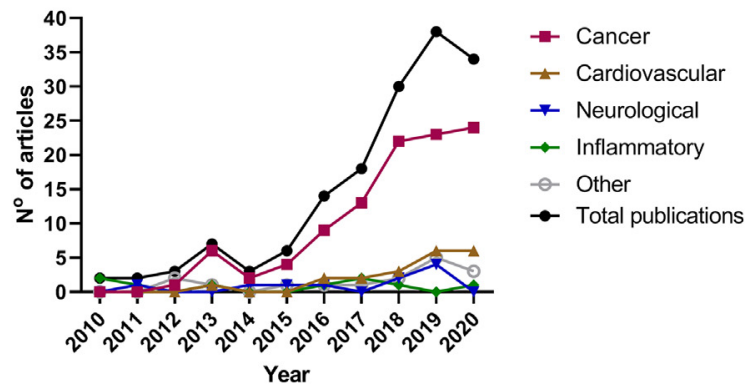

Fig. 4. Diseases tested in preclinical studies of EVs as drug delivery systems. Proportion (A) and trend (B) analysis of diseases/conditions investigated.

A

Animal models

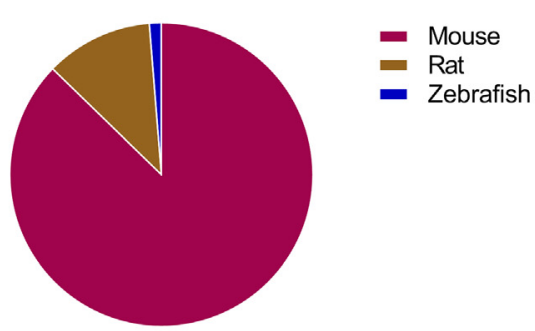

B

Trends in animal species

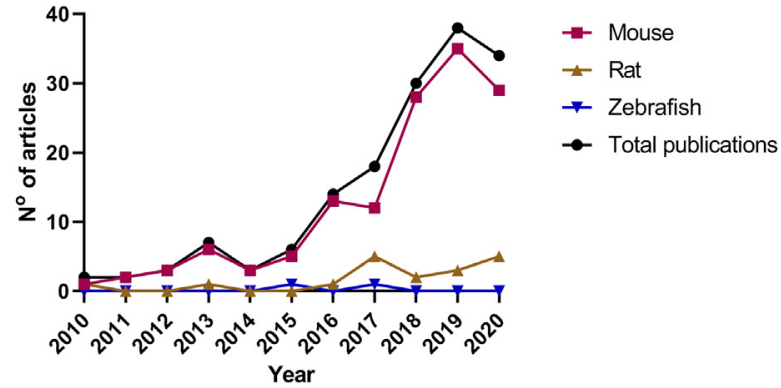

Fig. 5. Animal models in preclinical studies of EVs as drug delivery systems. Proportion (A) and trend (B) analysis animal models used.

A

EV donor cell types
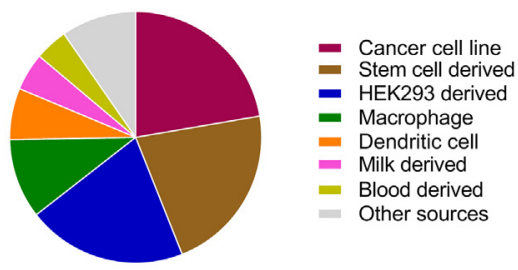

C

Cancer cell lines use by disease

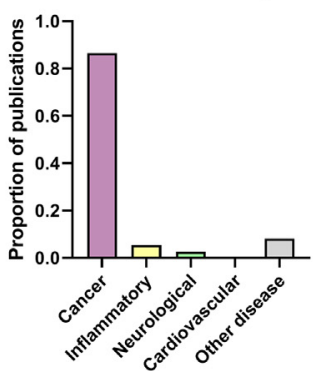

B

Trends in EV donor cell types

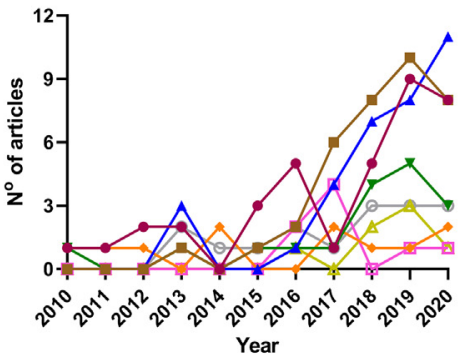

D

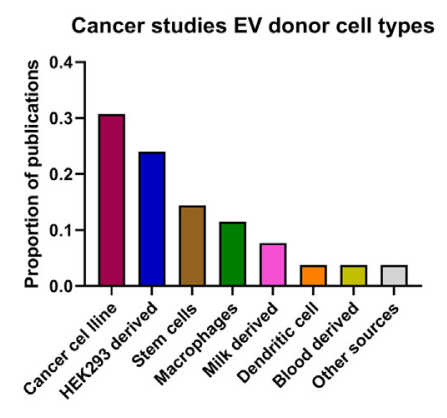

$$
\begin{aligned}
& \rightarrow \text { Cancer cell line } \\
& - \text { Stem cell derived } \\
& \rightarrow \text { HEK293 derived } \\
& \rightarrow \text { Macrophage } \\
& \rightarrow \text { Dendritic cell } \\
& \square \text { Milk derived } \\
& - \text { Blood derived } \\
& \rightarrow \text { Other sources }
\end{aligned}
$$

E

Cardiovascular disease EV donor cells

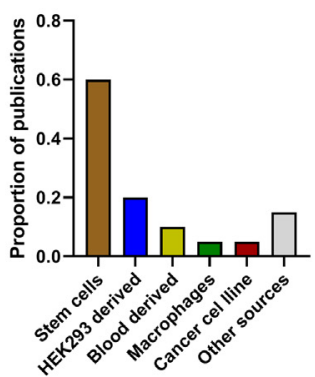

Fig. 6. EV donor cell types in preclinical studies of EVs as drug delivery systems. Proportion (A) and trend (B) analysis of EV donor cell types used. (C) EVs of cancer cell origin used in studies of various diseases. (D) Proportion of EV donor cell types used in cancer-related publications. (E) Proportion of EV donor cell types used in cardiovascular disease publications. 
delivery potential of EVs derived from blood (plasma or serum), milk or plant although the cellular origin of those EVs were mostly less defined (Fig. 6b). For studies using cancer cell-derived EVs, the vast majority (87\%) were focused on the treatment of cancer (Fig. 6c), likely due to the assumed tropism of cancer cell-derived EVs towards tumors $[105,216]$. Other frequently used donor cells in cancer related studies were HEK293 cells (24\%) and stem cells (14.4\%) (Fig. 6d). In studies of cardiovascular diseases (Fig. 6e), stem cell-derived EVs were commonly used (60\%).

\subsubsection{Active pharmaceutical ingredients (API) associated with EVs}

Among all the APIs loaded into or onto EVs in the analyzed preclinical studies (Fig. 7a,b), nucleic acids (46.5\%) and small molecule drugs (39.5\%) were the most popular, followed by proteins/peptides (17.8\%), oncolytic viruses (2.5\%) and nanoparticles (1.9\%). Of note, three nanoparticles, $\mathrm{Bi}_{2} \mathrm{Se}_{3}$ nanodots/DOX [123], PMA/AuBSA@Ce6 nanoparticles [103] and superparamagnetic iron oxide nanoparticles/Curcumin [49] were considered as APIs for this analysis since they were encapsulated into EVs as therapeutics. As the most popular API, the studied nucleic acids (Fig. 7c,d) were mainly miRNA (39.7\%), siRNA/shRNA (30.1\%), antagomiR (11\%), mRNA (9.6\%), and CRISPR/CAS9 (6.8\%). The other nucleic acids (9.6\%) encompassed plasmid DNA, DNA aptamer, lncRNA, CpG DNA and minicircle DNA. Interestingly, miRNA was particularly used for inflammatory disease and cardiovascular disease, constituting $55.6 \%$ and $40 \%$ of the respective publications (Supplementary Fig. S1a). siRNA/shRNA, however, was frequently used as an API in the studies (40\%) of neurological disease (Supplementary Fig. S1a). Of notice, siRNA/shRNA, antagomiR, mRNA and CRISPR/ CAS9 used as APIs were mainly reported in cancer studies, whereas miRNA as an API was used more often for other types of diseases (Supplementary Fig. S1b).

\subsubsection{API loading procedures}

APIs can be associated with EVs either before or after EV isolation. Before EV isolation, APIs were loaded via incubation, transfection-based methods or ultraviolet irradiation (Fig. 8a), whereas after EV isolation (Fig. 8b) APIs were loaded by either physical (electroporation, plain incubation, sonication, etc.) or chemical procedures (transfection kit, mix with organic solvent, etc.). Interestingly, we found no obvious preference of API loading before versus after EV isolation in the past decade (Supplementary Fig. S2). API loading before EV isolation was mainly achieved through transfection-based methods (77.9\%), including viral transduction, plasmid, miRNA, and antagomiR transfection (Fig. 8a). The frequency of API loading before EV isolation using plain incubation (11.7\%) and incubation post UV irradiation of the cells $(10.4 \%)$ is similar. As shown in Fig. 8b, API loading after EV isolation was mainly performed with physical procedures like electroporation (39\%), plain incubation (29.3\%) and sonication (12.2\%); and less than $20 \%$ studies employed chemical procedures such as transfection $(9.8 \%)$ and mixing with organic solvents (7.6\%). For the past decade, the use of electroporation has been increasing and reached $58.8 \%$ in the year 2020 among all studies involving API loading after EV isolation (Fig. 8b). Surprisingly, only nucleic acids (59.4\%) and small molecule drugs (40.6\%) were the APIs loaded by electroporation (Supplementary Fig. S3).

\subsubsection{Surface modification status}

Surface modification of EVs, and nanoparticles in general, with targeting ligands and other molecules represents a well-known and widespread method for targeting cells in organs and tissues of interest. Digging deeper into the targeting ligands used in EVs up to date, they were extensively discussed in a recent review by Gudbergsson et al., i.e., the most common type of targeting ligands used in EVs as DDS are small peptides (38\%), transmembrane proteins (34\%) and antibody fragments (25\%) [217]. For EVs as DDS in preclinical models, similar numbers of studies used surface-modified and surface-unmodified EVs although more studies intended to use EVs without surface modification in recent years (Fig. 9a,b). Despite PEGylation is commonly used for liposomes and other non-cell derived DDS to increase the circulation time, we found that only two recent studies (1.3\%) using EVs as DDS in preclinical models incorporated PEG to modify EV surface
A

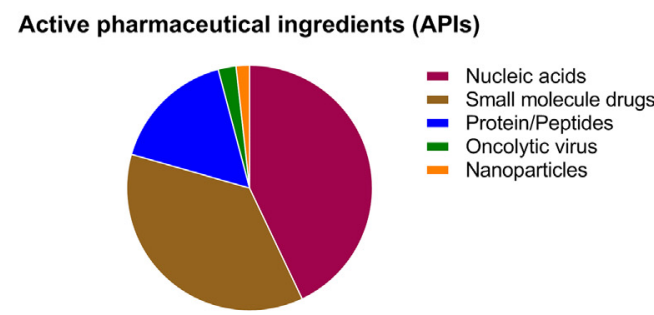

C

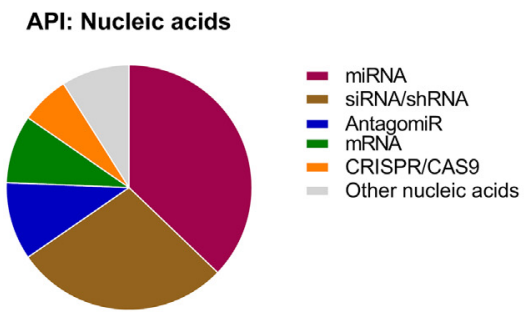

B

Trends in active pharmaceutical ingredients (APIs)

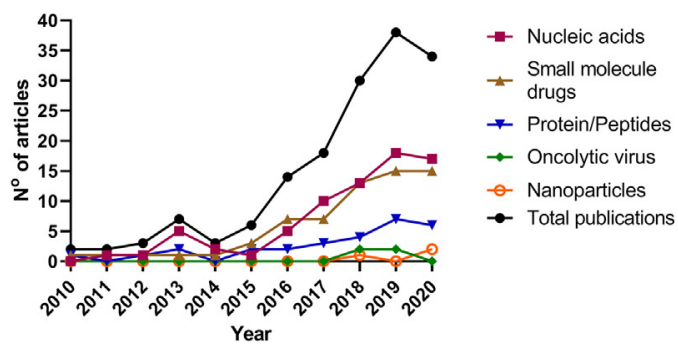

D

Trends in nucleic acids loaded

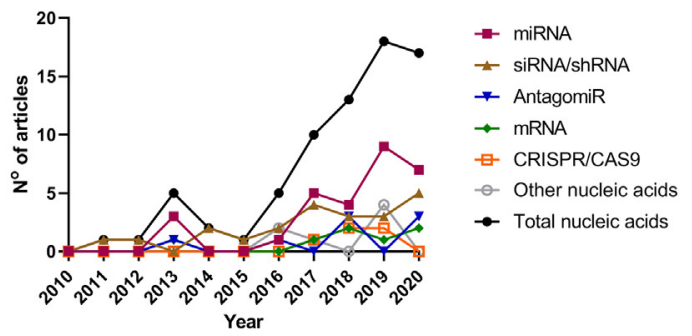

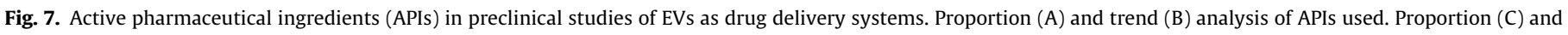
trend (D) analysis of the subtypes of nucleic acids used as APIs. 
A

API loading procedures before EV isolation

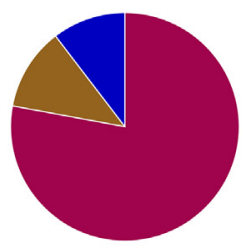

- Transfection based

- Incubation

- Ultraviolet irradiation
Trends in API loading procedures before EV isolation

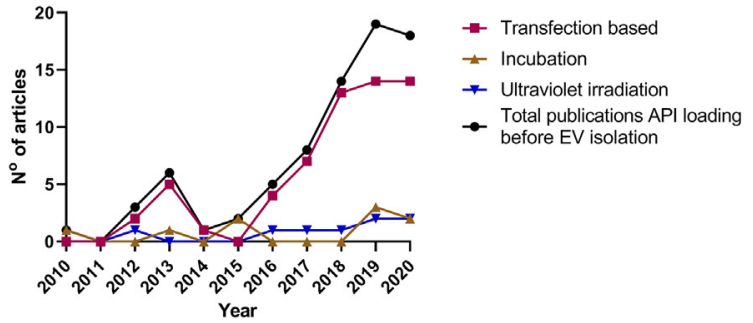

B

API loading procedures after EV isolation

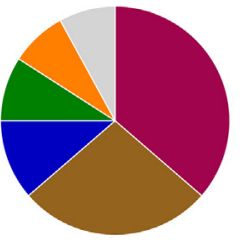

- Physical: Electroporation

Physical: Plain incubation

- Physical. Sonication

Chemical: Transfection kit

Chemical: Mix with organic solvent

Other

Total $=\mathbf{8 2}$

Trends in API loading procedures after EV isolation

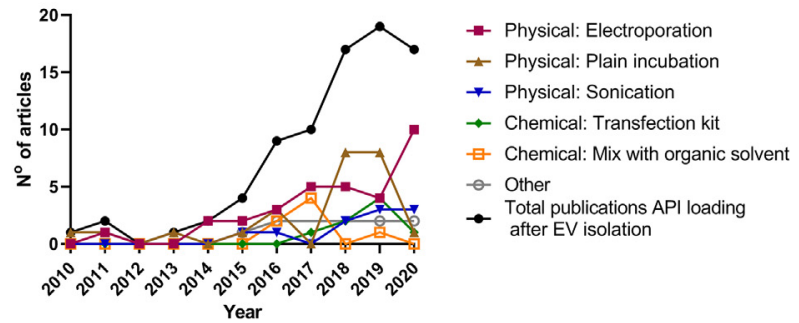

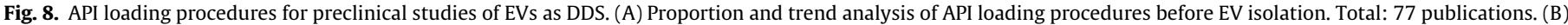
Proportion and trend analysis of API loading procedures after EV isolation. Total: 82 publications.

A

EV surface modifications

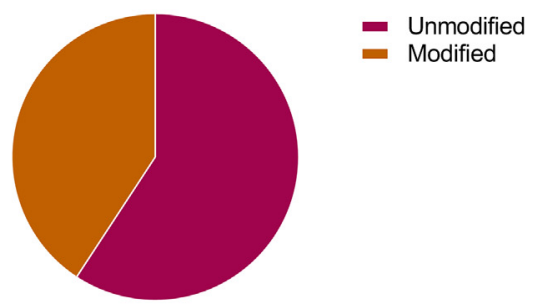

C

Proportion of surface modifications in EVs by disease

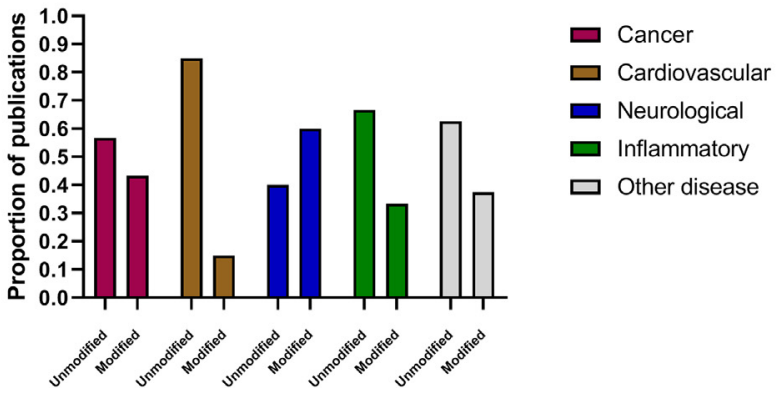

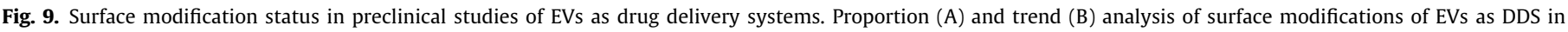
preclinical studies. (C) EVs with or without surface modifications used as DDS in preclinical studies of various diseases.

[81,129]. We further investigated the application of EV surface modifications in different disease studies (Fig. 9c). We found that $85 \%$ of cardiovascular disease studies used EVs without surface
B

Trends in surface modifications

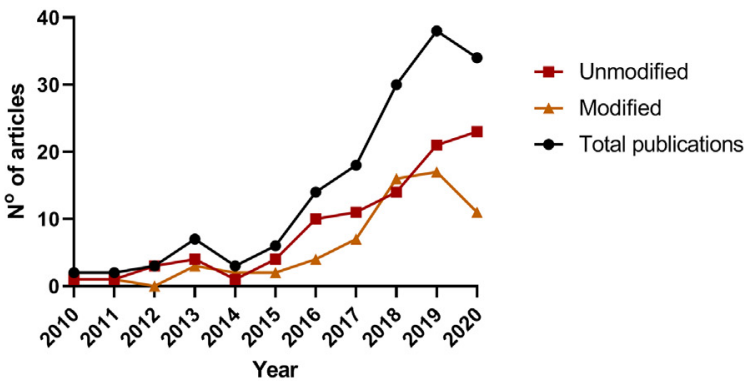


lar disease studies (Supplementary Fig. S4). In contrast, $60 \%$ of the neurological disease studies used EVs with surface modifications, mainly featuring the addition of the RVG peptide (83.3\%) that presumably facilitates transcytosis of EVs across the blood brain barrier (BBB) via targeting the alpha-7-subunit of the nicotinic acetylcholine receptor found in the brain $[180,218]$.

\subsubsection{Size and zeta potential}

Measuring the size and size distribution of nanoparticles is crucial for assessing the stability and drug delivery efficiency of a DDS. EVs are notorious for their high heterogeneity in size, which is influenced by multiple factors, including cell sources, cell culture conditions or EV producing microenvironment, and EV isolation procedures [219]. In addition, most of the current methodologies have various technical limitations and are not able to detect the smallest EVs (bellow $30 \mathrm{~nm}$ ), making it particularly difficult to compare EV parameters such as concentrations, size and size distribution [220]. In our systematic analysis (Fig. 10a,b) we observed that the majority of publications used nanoparticle tracking analysis (NTA) and dynamic light scattering (DLS) to determine EV size, representing $47.1 \%$ and $38.9 \%$ of total publications, respectively. Importantly, the utilization of these two methods keeps increasing in the recent years. DLS is broadly used to measure nanoparticles of a wide range of sizes, however, it suffers from severe limitations of accurately measuring the size or size distribution of polydisperse samples that contain mixed particle populations. In contrast, NTA, obtains size information based on the Brownian motion of individual particles. Given the high heterogeneity of isolated EVs, NTA outperforms DLS and therefore is recommend for measuring EV size by the International Society for EVs [8,221-223]. 12.7\% of the publications, to our surprise, did not measure EV size or size distribution. Furthermore, although most studies used transmission electron microscopy (TEM) to confirm EV size (as a secondary readout to DLS or NTA) while examining EV morphology, $5.7 \%$ of total studies only used TEM to estimate EV size. However, the vacuum pressure in TEM and sample preparations including fixation and dehydration probably affect the size of EVs [220]. Other EV size measurement methods, namely Tunable Resistive Pulse Sensing (TRPS) and flow cytometry acquainted for $1.3 \%$ and $0.6 \%$ of the publications, respectively.

Zeta potential, determined by DLS, which measures the surface charge of nanoparticles, is another important parameter affecting the stability, potential of aggregation and drug delivery effectiveness of EVs used as DDS. However, only $21.7 \%$ of the publications reported zeta potential of EVs (Fig. 10c). This means that nearly half of the studies using DLS for EV size measurement did not determine zeta potential at the same time. Nevertheless, among those studies that determined the zeta potential of EVs (Fig. 10d), $23.5 \%$ reported a neutral surface charge (between 0 and $-10 \mathrm{mV}$ ) and $76.5 \%$ reported a negative surface charge (zeta potential below $-10 \mathrm{mV})$.

\subsubsection{Estimation of purity}

Purity has become a critical issue to develop EVs as DDS due to the complex biological origin of EVs and the existing challenges in isolation procedures. As a drug delivery platform, low purity of EVs may compromise the drug loading efficiency and cause unexpected toxicity [224]. However, purity assessment is difficult. For instance, NTA cannot distinguish EVs from other particles such as LDL and protein complexes [225]. TEM allows the distinction of EVs from other non-EV particles [225,226], however, it is not able to quantify soluble contaminants in the sample [227]. Another informative strategy is using western blotting to semi-quantitatively examine the presence of EV proteins and known non-EV markers (e.g., calnexin) [227]. In this systematic review, we identified 8 studies that assessed EV purity according to the MISEV2018 guidelines, i.e., reporting quantitative ratios of protein:particle, lipid:particle or lipid:protein as an indicator of the purity of EVs [8], representing only $5.1 \%$ of the reviewed publications (Fig. 11a). In fact, all those studies reported the ratio of protein:particle rather than ratios of lipid:particle or lipid:protein. Excitingly, we observed an increasing trend in the number of publications that evaluated EV purity by reporting protein:particle ratio over recent years (Fig. 11b), especially after the publication of the latest MISEV guidelines in
A

EV size measurement
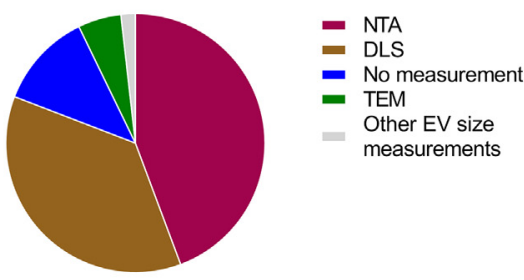

C
Trends in EV size measurement

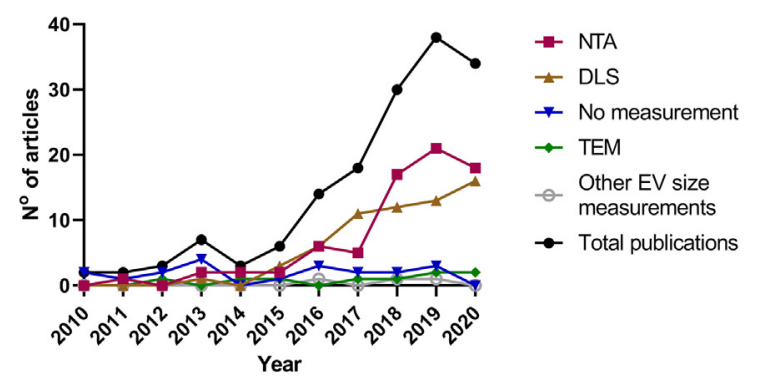

D

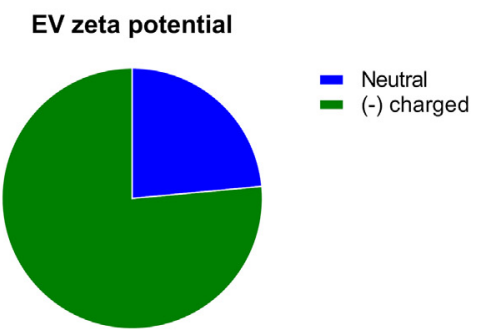

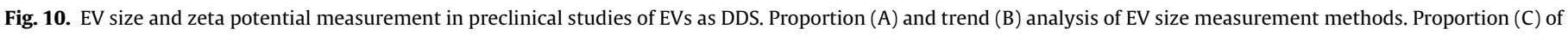
studies reporting EV zeta potential and qualitative analysis of the reported zeta potential (D). 
A

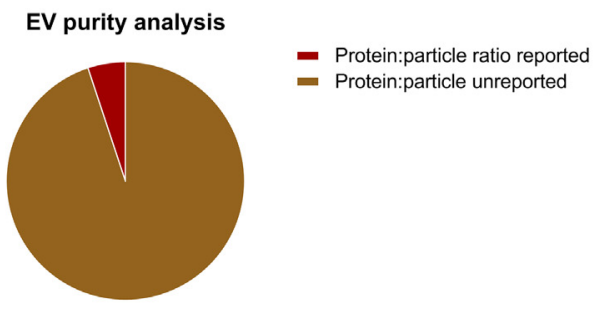

B

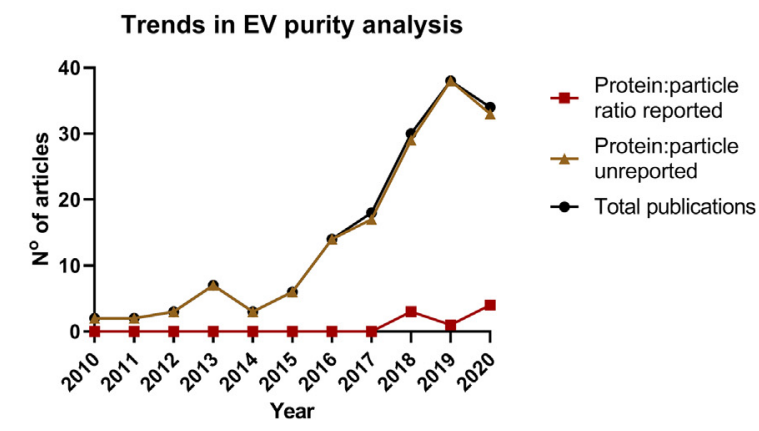

Fig. 11. Purity analysis of EVs as DDS in preclinical studies. (A) Proportion and (B) trend analysis of studies reporting EV purity with protein:particle ratio.

2018. Still, the applicability of this methodology is an issue and impurities in the form of protein aggregates [226] or non-EV particles of a similar size (e.g., LDL and VLDL) [228] cannot be discriminated. To address those issues, other methodologies such as flow cytometry detecting EV-specific markers (e.g., CD63, CD81, CD9) $[148,151,229,230]$ may be combined with the current ones. All in all, our findings point out the lack of consensus of a "gold standard" to determine EV purity.

\subsubsection{Biodistribution studies}

Monitoring biodistribution is an essential step in preclinical studies to elucidate the final destination of the administered EVs, thus providing valuable information on their targeting efficiency, pharmacokinetics and potential toxicity. Among all the preclinical studies of EVs as DDS included in our systematic analysis, $49.7 \%$ performed biodistribution experiments (Fig. 12a). Despite the number of studies reporting EV biodistribution has been increasing over the past decade, the change in the percentage of such studies is not impressive (Fig. 12b). The higher proportion of studies reporting EV biodistribution in 2018 (66.7\%) and 2020 (55.9\%) suggests that this issue is gaining attention in the field. Furthermore, we noticed that two methodologies were commonly used to investigate biodistribution EVs as DDS: tracing EVs labeled with fluorescence probes via in vivo optical imaging [80,184], or quantitative profiling the delivered cargoes in various animal organs ex vivo using analytical chemistry methods (e.g. HPLC or LC-MS) [75]. Given the possible disassociation between the vector and the cargo, combination of the two methodologies to monitor the biodistribution of both EVs (DDS) and delivered cargoes is recommended for future studies.

\subsubsection{Administration routes}

The administration routes of EVs as DDS in preclinical studies are important as they play a critical role in the pharmacokinetics and pharmacodynamics of drug-loaded EVs. Some studies have given evidence that tissue distribution and clearance rate of EVs might be influenced by the route of administration [227]. For this purpose, we evaluated the different administration routes of EVs that were used in the selected publications. Intravenous administration has been the dominant route of administration over the past decade (63.1\%), gaining particular popularity in recent years (Fig. 13a,b). In 2020, 88.2\% of all the publications used intravenous administration to apply EVs for drug delivery studies (Fig. 13b). Examining the routes of administration for each of the disease groups (Supplementary Fig. S3) shows that the main administration routes for cancer studies are intravenous (64.4\%), local (19.2\%) and intraperitoneal (11.5\%) administration, while for cardiovascular diseases they are intravenous (55\%) and local (40\%) administration.

\subsection{Clinical trials of EVs as DDS: On the way to reach patients}

A clear reflection of the promising therapeutic results accomplished with EVs in preclinical studies is the appearance of multiple clinical studies involving drug delivery through EVs in patients (Table 3).

Already five clinical trials have been using EVs as drug delivery systems in patients. Two of those clinical trials are in Phase I, one is in Phase I/II and the other two are in Phase II. In line with what can be seen from preclinical studies of the past decade, where $66.2 \%$ of the articles correspond to cancer studies, $80 \%$ of the clinical trials
A

EV biodistribution studies

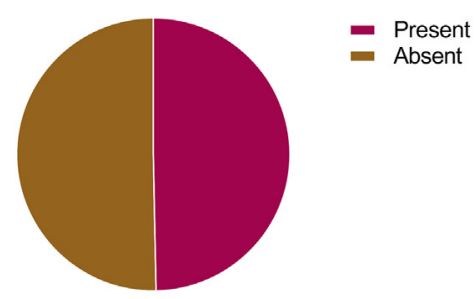

B

\section{Trends in EV biodistribution studies}

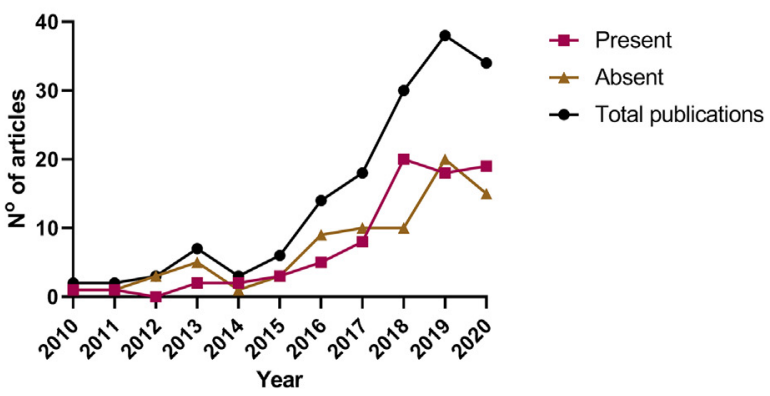

Fig. 12. EV biodistribution in preclinical studies of EVs as DDS. Proportion (A) and trend analysis (B) of studies reported EV biodistribution. 
A

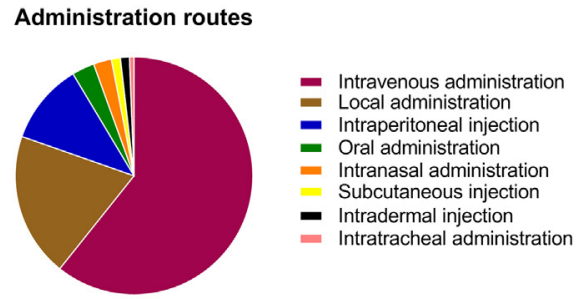

B

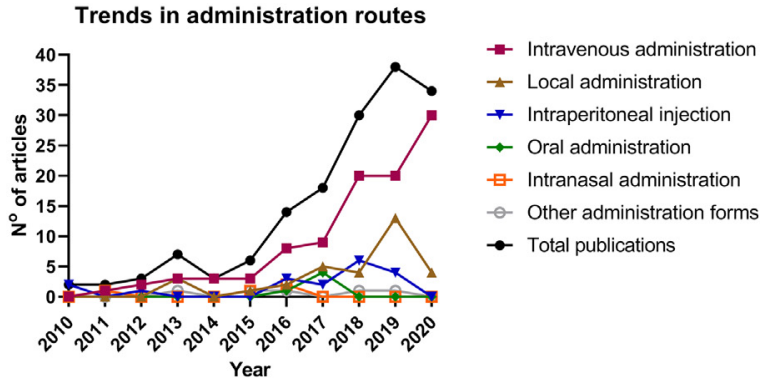

Fig. 13. Administration routes in preclinical studies. Proportion (A) and trend (B) analysis of administration routes of EVs as DDS in preclinical studies.

Table 3

Clinical trials of EVs as DDS.

\begin{tabular}{|c|c|c|c|c|c|}
\hline Disease & EV donor cell type & Compound & $\begin{array}{l}\text { Phase, } \\
\text { Enrollment }\end{array}$ & Status & NCT No. \\
\hline Acute ischemic stroke & $\begin{array}{l}\text { Mesenchymal stem } \\
\text { cells }\end{array}$ & MiR-124 & Phase I/II, 5* & $\begin{array}{l}\text { Recruiting (Estimated Study Completion Date: March } 17 \text {, } \\
\text { 2021) }\end{array}$ & NCT03384433 \\
\hline Pancreatic cancer & $\begin{array}{l}\text { Mesenchymal stem } \\
\text { cells }\end{array}$ & $\begin{array}{l}\text { siRNA } \\
\text { KrasG12D }\end{array}$ & Phase I, 28* & $\begin{array}{l}\text { Not yet recruiting (Estimated Study Completion Date: } \\
\text { March 2022) }\end{array}$ & NCT03608631 \\
\hline Colon cancer & Plant cells & Curcumin & Phase I, 7 & $\begin{array}{l}\text { Active, not recruiting (Estimated Study Completion Date: } \\
\text { December 2022) }\end{array}$ & NCT01294072 \\
\hline $\begin{array}{l}\text { Malignant ascites \& Pleural } \\
\quad \text { effusion }\end{array}$ & Tumor cells & $\begin{array}{l}\text { Chemothera- } \\
\text { peutics }\end{array}$ & Phase II, 30* & $\begin{array}{l}\text { Unknown }{ }^{1} \text { (Estimated Study Completion Date: March } \\
\text { 2014) }\end{array}$ & NCT01854866 \\
\hline Malignant pleural effusion & $\begin{array}{l}\text { Autologous tumor } \\
\text { cells }\end{array}$ & Methotrexate & Phase II, 90* & $\begin{array}{l}\text { Recruiting (Estimated Study Completion Date: December } \\
\text { 2019) }\end{array}$ & NCT02657460 \\
\hline
\end{tabular}

1 Study has passed its completion date and status has not been verified since September 2013.

* Estimated enrollment.

(4/5) are focused on cancer treatment. The remaining clinical trial aims to treat patients with acute ischemic stroke. Also, in preclinical studies cardiovascular diseases were second during the past decade (12.7\%). Cancer cells and stem cells represent the two main EV donor cell types, similar to that in preclinical studies, in clinical studies ( $40 \%$ of the totality each). Moreover, the types of APIs loaded in EVs, including small molecule drugs, miRNA and siRNA represent $60 \%, 20 \%$ and $20 \%$, respectively, of the five clinical trials, also similar to the trends observed in preclinical studies $39.5 \%$, $18.5 \%$ and $14 \%$, respectively). Although it is difficult to reach a statistically meaningful conclusion with only five clinical trials, the interesting similarities observed between clinical and preclinical studies as discussed above may not be coincidental. Along with the recent increase in pre-clinical and clinical trials using EVs as drug delivery systems, up to date there are 20 other clinical trials based on the intrinsic therapeutic function of unmodified EVs (Supplementary Table S2). We consider that it is of vital importance to establish a multifactorial comparison between the current gold standard of nanomedicine, the liposomes, and EVs, to achieve a meaningful assessment of the possible niches where to potentially use EVs as DDS.

\section{Liposomes versus EVs for drug delivery: heads up}

\subsection{Short overview of EVs as therapeutic delivery vehicles in comparison with liposomes}

The main challenges in delivery of therapeutics to the site of action are off-target toxicity, rapid clearance, and low accumulation and bioavailability in target tissue, cell or organelle [229]. To circumvent these challenges, a broad range of synthetic delivery vehicles (liposomes, lipid nanoparticles, polymeric micelles, inorganic nanoparticles, dendrimers, etc.) have been developed in the last few decades with some of them already clinically approved.
The main concept of a drug delivery vehicle is that the tissue distribution and the body clearance are governed by the characteristics of the vehicle instead of those of the drug itself [230-232]. Out of the available spectrum of all nanoparticles, the most successful delivery vehicles up to date, with the highest number of clinical approvals on the market, are the liposomes. Given their similarity, a side-by-side comparison of EVs with liposomes, regarding their physicochemical properties and their drug delivery capacity, is presented next (Fig. 14).

\subsection{Physical features, production and quality control}

Liposomes are structurally similar to EVs as they are composed of a lipid bilayer around an aqueous compartment [233]. Similarly, EVs can carry hydrophobic drugs within the lipid membrane bilayer and hydrophilic drugs in the aqueous core [234]. In addition, the dimensions of the clinically approved liposomes are around $100 \mathrm{~nm}$ [235-239], similar to exosomes and microvesicles [8,53]. This size is compatible with avoidance of premature clearance by macrophages, which increases with increasing particle size, and renal clearance, which occurs to particles with hydrodynamic radius lower than 5-6 nm [240,241]. Moreover, the size of these liposomes allows extravasation at certain body sites after intravenous administration and uptake by cells [242,243]. Despite the similarities they share, a number of differences exist between liposomes and EVs as drug delivery vehicles (Fig. 14). This can be illustrated by comparing EVs with the pioneer liposomal formulation of Doxil ${ }^{\circledR} /$ Caelyx $^{\circledR}$, PEGylated liposomes loaded with the chemotherapeutic doxorubicin [244]. Being approved by the FDA in 1995 and the European Medicines Agency (EMA) in 1996, it was the first nanoparticular drug brought to the market. Compared to EVs, clinically used liposomes like Doxil ${ }^{\circledR}$ are composed of a limited number of defined lipids but no cellular components such as proteins and genetic materials [245], and, therefore, they are rela- 
A

\section{PEGylated liposomes}

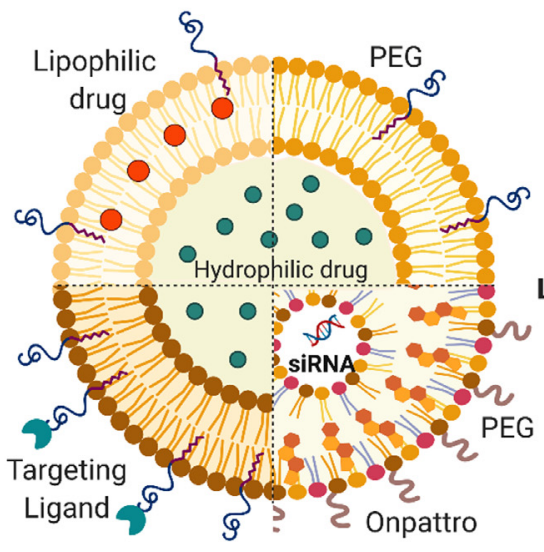

Mainly rely on passive targeting Active targeting via ligands or protein corona
B

\section{Extracellular vesicles}

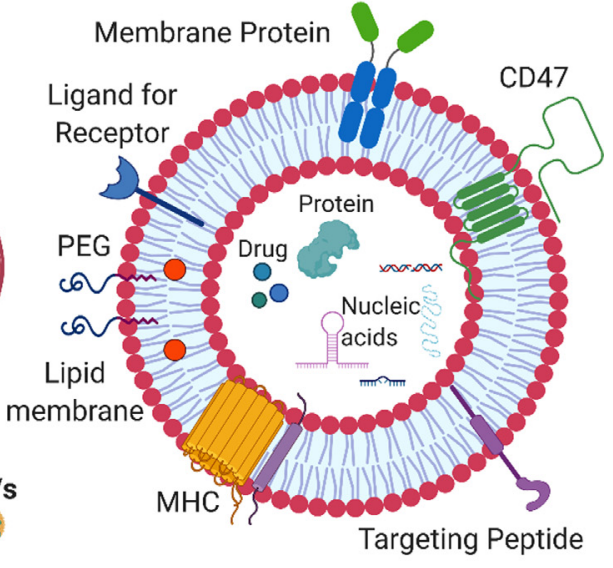

Interactive surface allow active targeting Passive targeting still present

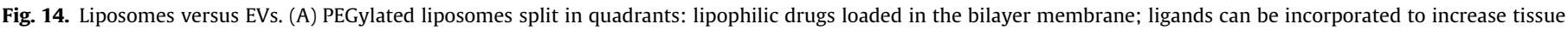

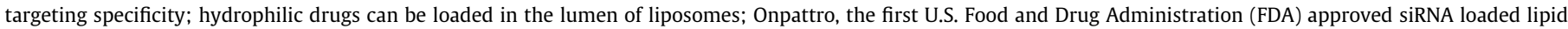

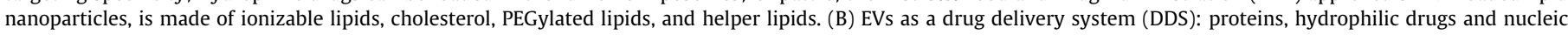

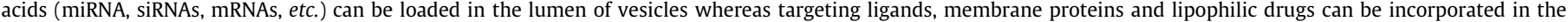
membrane.

tively easy to handle in pharmaceutical quality control and largescale manufacture processes. While the initial Doxil ${ }^{\circledR}$ contained naturally sourced phospholipids, like hydrogenated soy phosphatidylcholine, the current formulation only uses synthetic phospholipids. A variety of high throughput methods exist to manufacture liposomes, including extrusion, ethanol injection and microfluidic mixing. Because of the self-assembly of liposomes from all the individual components, full control exists over the composition, including the constitution of the aqueous phase. This is exploited in the Doxil ${ }^{\circledR}$ formulation by loading a high concentration of ammonium sulphate in the liposome interior. The gradient of this salt over the membrane serves as the driving force for the subsequent remote loading of doxorubicin. Similarly, in Vyxeos ${ }^{\circledR}$, the exact proportion of two co-encapsulated drugs (daunorubicin and cytarabine) can be controlled during production to obtain the optimal ratio for synergistic action.

EVs, however, contain the full repertoire of cellular lipids and are particularly enriched in lipid raft components like sphingomyelin, cholesterol and lysophospholipids. It is noticeable that a higher degree of complexity can be achieved with EVs in comparison with mixing individual components in liposomes. Furthermore, due to the presence of biomolecules in the membrane and core, additional binding pockets may be present in EVs for drug loading. However, loading into pre-formed EVs is extremely challenging [49]. This presents a challenge for manufacture and quality control as the particular EV composition is a snapshot of the cell at the time of production which may vary from cell to cell, from culture condition to culture condition and over time. In terms of production and harvesting of EVs, so far scale-up remains highly challenging.

\subsection{In vivo administration of EVs and liposomes}

\subsubsection{Nanoparticles (EVs E liposomes) are rapidly cleared by the mononuclear phagocyte system (MPS)}

Liposomes represent biodegradable and biocompatible DDS with exceptionally versatile high-throughput preparation and drug encapsulation efficiencies, allowing lyophilization and surface modifications [246]. However, there are still some obstacles that need to be overcome to obtain improved drug efficacy, diminished drug toxicity and reduced off-target effects. One of the biggest hurdles to obtain optimal drug delivery to the target tissue is the rapid systemic clearance of liposomes from the blood through the mononuclear phagocyte system (MPS) mediated by the opsonization of the liposomes (surface adsorption of serum proteins) and the subsequent phagocytosis by macrophages, mainly those in the liver and spleen [247]. Apart from macrophages, other cells of the reticuloendothelial system (RES), such as liver sinusoidal endothelial cells, have also been reported to take up and clear liposomes [248,249].

In a similar fashion, EVs are rapidly cleared by the MPS after systemic administration in vivo, notwithstanding their composition [250,251]. Likewise, after intravenous injection of EVs in vivo, the biodistribution profile of EVs resembles that of nonpegylated liposomes of the same size and surface charge [252]. In order to reduce immunogenicity and to avoid rapid blood clearance of liposomes, polyethylene glycol (PEG) surface coating is widely used, thereby enabling more accumulation in the target tissue [253]. The decoration of EVs with PEG or PEG-coupled targeting ligands has been proposed as a promising strategy to enhance EV drug delivery capacities $[81,129,254]$. Another interesting strategy is the selection of subsets of EVs that contain specific surface proteins like CD47. This protein acts as a "don't eat me signal" in EVs and may confer them the ability to circumvent the MPS and exhibit longer circulation time [148,255]. Furthermore, transiently blocking the RES through systemic administration of empty liposomes or other substances like dextran sulfate has been explored to obtain a substantial increase in functional drug delivery and tumor accumulation for both drug-loaded liposomes and EVs [256-258].

\subsubsection{Accelerated blood clearance $(A B C)$ phenomenon upon multiple injections of nanoparticles}

ABC was first reported by Dams et al. by showing that PEGylated liposomes exhibited enhanced clearance and loss of effectiveness upon repeated administration [259]. This is due to their opsoniza- 
tion by anti-PEG IgM antibodies and subsequent complement activation response [260,261], which accelerates the recognition and clearance of successive injections of PEGylated liposomes particularly by liver macrophages. Strategies that have been reported to possibly circumvent the $A B C$ phenomenon include increasing the lipid dose for the first injection or administering a small pre-dose before the second liposomal injection [262]. Similarly, PEG chain modification of the EV surface is a common method used to prolong the circulation time of EVs [254], and it is reasonable to assume that the $A B C$ effect also applies to PEG-coated EVs and subsequently affects the effectiveness of EVs, since the occurrence of the ABC effect in case of other PEGylated non-liposomal nanoparticles has been reported as well [263,264]. Different strategies could be applied in order to circumvent potential $A B C$ effect in EVs by: 1) using substitutions of PEG chains [260,265]; 2) administering immune suppressive agents [260] or 3) applying modified anti-PEG Ab to compete with the natural anti-PEG Ab [266].

\subsubsection{Complement activation-related pseudoallergy (CARPA) upon nanoparticle injection}

CARPA is an acute allergic reaction with symptoms including cardiopulmonary, hemodynamic, and an array of other pathophysiological changes [267]. It has been reported that lipidic nanoparticles could trigger adverse immunological reactions, and result in hypersensitivity CARPA, hindering their clinical use in hypersensitive patients [268]. The severity of CARPA after systemic liposomal administration in patients is influenced by morphological properties, size, surface charge, PEGylation, and cholesterol content, amongst other characteristics of liposomes [268]. A representative example is Doxil ${ }^{\circledR}$, which has been reported to elicit rare but serious acute infusion reactions in cancer patients [269]. Therefore, CARPA has been recognized as a safety issue. Although a number of studies have confirmed the administration of liposomes could result in adverse CARPA reactions, its exact mechanism is still unknown. At the same time this provides an opportunity for investigating the application of EVs as DDS, but potentially without CARPA triggering. EVs may have the potential to overcome CARPA effects as some studies claimed that EVs consist of a natural cocktail of biomolecules that do not cause adverse reactions linked to liposomal particle infusion [270,271]. But still, regarding possible CARPA adverse reactions after administration of EVs, their occurrence remains uncertain. A Phase I clinical trial indicated that dendritic cell-derived EVs caused mild inflammatory reactions after subcutaneous administration in half of the patients [272,273]. However, in some studies using pig models, CARPA-related adverse effects were not observed after intracardiac administration of human MSC-derived EVs, while low doses of liposomes could induce shock-like symptoms $[46,47]$. Regarding the CARPA adverse reactions after administration of EVs, these remain uncertain and demand extensive research as the current experimental evidence is too limited $[46,47,270,273]$. Of note, it is generally accepted that the choice of species for preclinical validation is important to assess possible adverse reactions after infusion of EVs and liposomes [3]. CARPA adverse reactions are not that common in rodents as they are in humans and other species such as humanlike pigs [3]. Therefore, the latter might be more suitable for these types of studies.

\subsubsection{Biodistribution profiles: passive vs. active targeting}

All the approved liposomal drugs on the market rely on passive targeting, and only a small proportion of the actively targeted formulations have reached clinical stages. This is due to the fact that even with surface ligand-targeting a specific receptor on target cells, the accumulation of liposomes is still considered to be dictated by passive extravasation processes referred to as the enhanced permeability and retention (EPR) effect [274]. Regarding drug delivery in some types of cancer, liposomes take advantage of the EPR effect caused by the leakiness of the tumor vessels to achieve higher tumor accumulation [275]. Via the EPR effect, liposomes with longer circulation time are prone to accumulate in the tumor [234] or injured myocardium [276]. Interestingly, liposomes have also been suggested in a recent study to enter tumors through a process mediated by endothelial cells that line tumor blood vessels [277]. A better understanding of those pathways will help the development of more effective nanomedicine strategies.

In the case of EVs, studies have shown that EVs with a size below $100 \mathrm{~nm}$ are also capable of achieving enhanced tumor targeting through the EPR effect in some tumors [278,279]. Still, EVs naturally and inevitably exhibit an interactive surface which makes them natural actively targeted carriers. However, they display, in general, short circulation time which limits their opportunity to take advantage of passive targeting. Enrichment of "don't eat me" surface molecules such as CD47 that likely enable EVs to escape from the MPS clearance system [148] might help to engineer EVs for passive targeting. Of note though, the EPR effect is rather diversified being only present in some types of tumors and varies among individuals and tumor types and should not be regarded as a general characteristic of solid malignancies, as evidenced in clinical canine cancer patients [262]. Furthermore, the preclinical models used for cancer studies may not necessarily represent the EPR features of human tumors as they often have a different microenvironment and growth kinetics [280]. For preclinical testing of EVs as a DDS, these potential challenges need to be addressed and the data should be extrapolated to the human situation with caution as the EPR effect is less significant in the clinic than in rodents (especially in xenograft models) [281].

Certain EV subsets display organotropic or tumor-targeting capacities [282], and the targeting properties of EVs can be modified by genetic engineering of the donor cells [255]. From a biodistribution point of view, the majority of administered liposomes end up in the liver and spleen due to removal by the MPS. In a similar fashion, despites their interactive surfaces, passive biodistribution of EVs is a big challenge for drug delivery applications as the predominant uptake organs for EVs in the reported studies so far are also the liver and spleen [3]. Nonetheless, integrin expression profiles on EVs have been reported to directly relate to tissue organotropism to the lung, liver and brain [282]. Moreover, each tumor-derived EV population exhibits organotropism towards specific organs where EV parental cell lines originate [282]. These results reinforce the concept that selection of EV sources might be key to achieve specific tissue targeting [245,278].

\subsubsection{Pharmacokinetics and pharmacodynamics (PK/PD)}

PK/PD as a simulation system based on the drug's physiological and pharmacological effects can provide valuable information on the treatment efficacy of drugs [283]. Compared with free forms of drugs, encapsulation of drugs in liposomes prevents rapid clearance and remarkably changes the PK profile of drugs [284]. For example, systemic clearance of doxorubicin in humans is reduced from $45 \mathrm{~L} / \mathrm{h}$ to $0.1 \mathrm{~L} / \mathrm{h}$ and accumulation in targeted tumors is increased by 4 to 16 -fold upon encapsulation into liposomes, i.e. Doxil $^{\circledR}$ [285]. Liposomal amikacin, Arikayce ${ }^{\circledR}$, is another example of a marketed liposome suspension. Compared to intravenous administration of free amikacin in rats, inhalation of Arikayce ${ }^{\circledR}$ increases the concentrations of amikacin in the lung, airways, and macrophages by 42-, 69-, and 274-fold, respectively [286]. Compared with liposomes, EVs might have potential to reduce MPS-mediated clearance due to the presence of surface CD47 [148] but more evidence is demanded. EVs derived from five different cell types showed comparable physicochemical (particle size and zeta potential) and pharmacokinetic properties (biodistribution and EV time course in the serum) after injection into mice 
[287]. Unfortunately, very little information is available regarding $\mathrm{PK} / \mathrm{PD}$ properties of EVs, which is likely due to the challenge of large-scale EV production and the presence of endogenous EVs. A comprehensive understanding of the PK/PD properties of EVs as DDS is still missing but crucial for EVs to reach the clinic.

\section{Critical discussion}

Through the detailed, systematic analysis of the selected publications we obtained a global picture of the field of EVs as DDS and its future trends. These studies tested EVs as DDS primarily in murine cancer models after intravenous administration. The increasing number of publications using EVs as DDS in cancer studies relates to the potential that EVs harbor for tumor targeting, cancer vaccination/antigen presentation and their capacity to carry a vast array of therapeutic molecules such as nucleic acids and small molecule drugs. The majority of EV donor cell types used in those publications were cancer cells, stem cells and HEK293 cells, while the majority of APIs loaded into EVs were nucleic acid therapeutics and small molecule drugs. Regarding nucleic acids as APIs, these mainly corresponded to miRNA and siRNA/shRNA. This is in line with the general increase in their therapeutic use and suitability for EV loading demonstrated during the recent years. We found no obvious preference of API loading before versus after EV isolation and the loading of isolated EVs was done by electroporation in about one third of the studies. Moreover, nearly half of the studies used EVs subjected to surface modifications, leaving plenty of room for debate on whether such modifications are of significance for increasing the therapeutic efficacy of APIs but sacrificing simplicity and reproducibility. From our results we can also infer that the usage of NTA and DLS for measuring EV size and size distribution has been increasing in the recent years, whereas EV zeta potential is unexpectedly neglected in most studies. Furthermore, approximal half of the studies performed EV biodistribution analysis. Finally, but disappointingly, reports on the purity of tested EVs for drug delivery remain scarce.

\subsection{Seeing the glass half empty: Missing gaps for establishing EVs as effective and safe drug delivery systems}

After analyzing the preclinical studies on EVs as drug delivery systems, we have to conclude that essential information for a proper understanding of the behavior and effects of EVs used is missing in many of the publications. Information such as EV heterogeneity, size and charge range, characterization of EV intrinsic content, safety, robust biodistribution studies, calculations of final API loaded concentration, storage conditions or comparison with liposomal carriers is often not or only poorly reported.

\subsubsection{Challenges in production, isolation, purity and characterization}

Major challenges are production, isolation, purity and characterization. Biological production methods are by definition difficult to standardize. It requires full control over cell culture conditions, genetic stability of the culture and the presence of cellular subtypes or contaminants. Production of EVs is higher when cells are in suspension but not all cell types can be grown under such circumstances. The development of efficient bioreactors for EV production might offer a promising way of generating large amounts of EVs with the possibility to scale up the process for clinical purposes. Even then, therapy with EVs will remain a costly drug delivery strategy. Well-established isolation methods like ultracentrifugation represent a hassle for large-scale production of undamaged EVs; other methods such as tangential flow filtration (TFF) might be more suitable for this purpose [234]. EV samples are still a mixture of bilayered vesicles and diverse lipoprotein particles in the vast majority of the studies we systematically reviewed, with little or no indication given on the level of EV purity. The lack of EV purity in the isolated fractions is of big concern and hampers their clinical translation. In addition, EV heterogeneity has hindered their characterization at the subtype level, as well as subclass-specific modification of properties and biological activity [15-18]. The lack of characterization, in turn, poses challenges in the interpretation of biodistribution and intracellular delivery results [288]. Moreover, due to this lack of characterization of EVs intrinsic content, additional attention is needed regarding safety. A clear risk example would be the use of EVs derived from cancer cell lines as drug delivery systems, even though such EVs have been reported to play a key role in malignant processes like the formation of pre-metastatic niches.

\subsubsection{Misleading information on EV biodistribution}

Another important challenge is the lack of standardized methods to study EV biodistribution. The use of fluorescently labeled lipids, or lipid conjugated dyes presents two fundamental problems: 1) imaging of a fluorophore or a tracer dye is not necessarily imaging of the EV itself as the labeling probes may drop off from the EVs. This phenomenon has been recently reported for liposomes [289]. Other labeling approaches (please refer to the comprehensive review by Gangadaran et al. [290]), including magnetic nanoparticles, radiolabeling, or indirect labeling via genetic modification of the parental cells to pack bioluminescent reporter proteins into or onto EVs, in combination with more sophisticated (but usually expensive) imaging modalities like magnetic resonance imaging (MRI), may confer more accurate biodistribution profiles of EVs. Similar to non-cell derived nanoparticles such as liposomes, majority of injected EVs accumulate in the liver, spleen and lungs regardless of their origin, likely due to recognition by the recipient's MPS [291]. However, a recent study that genetically labeled cardiomyocyte-derived EVs using a CD63NanoLuc reporter in transgenic mice demonstrated that endogenous EVs were mainly taken up by the thymus, testis, lung and kidney instead of the liver [292]. These discrepancies in biodistribution of endogenous and exogenous EVs indicate that EV isolation, storage, labeling strategy, or allogeneic-induced recognition of EVs by the MPS alter the biological behavior of EVs in vivo. Examining EV biodistribution has been considered as monitoring EV cellular uptake and therefore EV delivery of cargoes, however, cellular uptake of EVs might not correspond to the ultimate delivery of the cargo as the loaded cargo may escape or be leaked from the EVs. A possible strategy to solve this disconnection between cellular uptake of EVs and functional delivery of their cargoes would be the use of two labeling methodologies simultaneously (for cargo and EV membrane, respectively) to study the colocalization of two physiochemically distinct labels. Even then, cellular internalization of both EVs and their cargoes may not guarantee the successful delivery. This was recently reported by Reshke et al. as cellular uptake of GFP siRNA loaded EVs failed to knock down GFP in Kupffer cells in mice [178]. Thus, EV biodistribution data should be interpreted with caution.

\subsubsection{Complications in upscaling, loading strategies and storage}

In line with the previously mentioned issues regarding isolation and characterization of EVs comes the challenge of efficient upscaling of EVs for clinical use. Reproducibility remains an issue with batch to batch production [293] and this matter is also of great concern for academic research, which would benefit enormously from reproducible and reliable experimental conditions. To use EVs in drug delivery, the lack of effective loading strategies that do not compromise the structure and functions of EVs is problematic. Exogenous loading methods have been reported to give variable outcomes and loading efficiencies whereas for genetic 
engineering of the parental cells, loading capacities remain generally low [293-295]. Better understanding of the intracellular formation, release and trafficking processes, might improve API loading. Another aspect, which is often not mentioned in preclinical studies, is the storage condition for the EVs used. It is essential that storage conditions that assure EV functionality are used, in order to guarantee current Good Manufacturing Practices (cGMP) [18]. There is a general agreement by the scientific community to support storage of EVs at $-80^{\circ} \mathrm{C}[52]$. Cryoprotectants such as trehalose may be necessary to protect damage and aggregation of EVs induced by freezing/thawing during storage and recovery [296]. Notably, the study of EV stability under different conditions could be crucial to determine their feasibility for clinical use as not all the countries have the resources to guarantee $-80^{\circ} \mathrm{C}$ storage at clinical centers.

\subsubsection{Lack of comparison with liposomes}

Surprisingly, we found that as of today there are only very few publications that have done a head-to-head comparison in delivery efficiency of EVs versus liposomes [148,178,297] and from these some are comparing EVs to liposomes without giving sufficient information on the type of liposomes used [148]. Given the similarity in size, shape and basic membrane composition (phospholipids), to examine the possible advantages of EVs as a new class of DDS, it may be recommendable to include liposomal formulations as a control DDS in future studies. Liposomal systems could be used as a reference in order to investigate EV cell targeting, routes of cellular uptake and intracellular trafficking in recipient cells.

\subsection{Seeing the glass half full: accomplished landmarks of EVs for drug delivery}

As it can be inferred from our study (Table 2), a variety of approaches and biologic materials have been used in order to successfully use EVs as DDS in animal models. The constant increase in the number of publications involving EVs as DDS during the past decade is a promising augury for the future of the field. From the publications we systematically reviewed, some important landmarks have been achieved:

\subsubsection{Active targeting}

When it comes to active targeting, which is one of the main aims of using EVs as DDS, considerable progress has been achieved already with EVs. Intravenously injected tumor-derived EVs tend to target parental tumor tissues. Compared to the gold standard nanocarrier, Doxil ${ }^{\circledR}$, drug-loaded EVs showed increased therapeutic retention in tumor tissues and more pronounced tumor suppression in nude mice [151]. Multiple strategies exist to introduce active targeting in EVs, such as the generation of EVs decorated with rabies virus protein enabling targeting of EVs to the brain [180]. Similarly, EVs have been coated with nanobodies, bispecific antibodies or peptides to improve tissue tropism, for example to target regenerative exosomes to myocardial infarctions using a cardiac homing peptide [298]. Furthermore, a recent publication demonstrated that small EVs exhibit targeted delivery of siRNA to specific cell populations and tissues in mice [178].

\subsubsection{Improved circulation time}

As stated before, the short circulation time of EVs is a limiting factor for their drug delivery capacities and therefore several studies focused on increasing the circulation time of EVs, a strategy reminiscent of the liposome field. Addition of CD47 or PEG was shown to reduce clearance by macrophages of the RES [299]. Whereas unmodified EVs were rapidly cleared within 10 min upon systemic administration, EVs post-inserted with nanobody-PEG-
Table 4

Defining the niches for EVs based on their attributes as DDS when compared to liposomes.

\begin{tabular}{|c|c|}
\hline Liposomes & EVs \\
\hline $\begin{array}{l}\text { Starting materials - limited number } \\
\text { of chemically defined molecules }\end{array}$ & $\begin{array}{l}\text { Crossing barriers - ability to cross } \\
\text { biological barriers such as the BBB }\end{array}$ \\
\hline $\begin{array}{l}\text { Reproducibility - high control over } \\
\text { manufacture, loading and } \\
\text { physicochemical characteristics }\end{array}$ & $\begin{array}{l}\text { Trafficking - cell-specific interaction } \\
\text { and internalization routes through } \\
\text { rich biomolecular surface }\end{array}$ \\
\hline $\begin{array}{l}\text { Scalability - industrial scale } \\
\text { production methods have been } \\
\text { developed }\end{array}$ & $\begin{array}{l}\text { Complex cargo - can carry a mix of } \\
\text { bioactive molecules (e.g. cell surface } \\
\text { receptors, RNA species \& post- } \\
\text { translationally modified proteins) } \\
\text { simultaneously }\end{array}$ \\
\hline $\begin{array}{l}\text { GMP - entire production process can } \\
\text { be GMP grade }\end{array}$ & $\begin{array}{l}\text { Multifactorial modulation - can affect } \\
\text { diseases at multiple nodes of } \\
\text { intervention }\end{array}$ \\
\hline $\begin{array}{l}\text { Storage - long shelf live and } \\
\text { pharmaceutical stability }\end{array}$ & $\begin{array}{l}\text { Flexibility - opportunities for } \\
\text { extensive bioengineering }\end{array}$ \\
\hline
\end{tabular}

lipids could be detected in plasma for over 60 min post injection and exhibited improved cell specificity [254]. However, this circulation time is still very short in comparison with PEGylated liposomes. Being optimistic, there is some evidence that the satisfactory performance of EVs with respect to plasma stability might upgrade their therapeutic potency [217].

\subsubsection{Upgraded loading efficiency}

Drug loading remains one of the biggest hurdles to develop EVbased DDS. Interestingly, EVs seem to be suitable for loading and delivery of RNA-based therapeutics. In a recent study, siRNA was integrated into a pre-miRNA backbone in order to load it more efficiently into EVs [178]. Notably, those EVs achieved similar gene knockdown efficiency with over ten-fold less siRNA than the typically required dose for lipid nanoparticles [178]. Another recent study from our own group, which used a CRISPR/Cas9-based RNA transfer reporter system [300], suggests that there is a difference of several orders of magnitude regarding more potent delivery of sgRNA compared to liposomal systems [297]. An emerging strategy to improve drug loading efficiency while maintaining the unique features of EVs is to generate hybrid EV systems by incorporating components such as liposomes into EVs [301] or EV mimetics [302]. While developing methodologies for improving drug loading efficiency into EVs, EV mimetics or hybrid EV systems, as discussed in a recent article [55], heterogeneity of EVs in molecular composition, surface features, and subpopulation (in terms of cell origin, size, surface charge, etc.) may also impact EV drug loading and delivery.

\subsubsection{EVs in intracellular trafficking: escaping the endosomal system}

The delivery of RNA through nanoparticles is well known to be mediated through the endolysosomal system at the subcellular level. This might represent a bottleneck for efficient RNA delivery, as only $1-2 \%$ of the cargo escapes into the cytosol [303]. In this sense, there might be differences between intracellular routing of EVs and other nanoparticles, in light of emerging evidence suggesting that EVs are capable of escaping endosomal degradative pathways [178,255,297,304,305]. Elucidating the details of the EV cargo delivery process might be key to upgrade the delivery of therapeutic molecules through EVs when compared to other nanoparticulate systems [255,297].

\subsubsection{Crossing the Blood-Brain barrier (BBB)}

It has been shown that $98 \%$ of small molecule drugs, and $\sim 100 \%$ of biologic drugs cannot cross the blood-brain barrier (BBB) [302]. Therefore, it is understandable that to date there is not a single nanoparticle formulation approved by the U.S. FDA that manages 


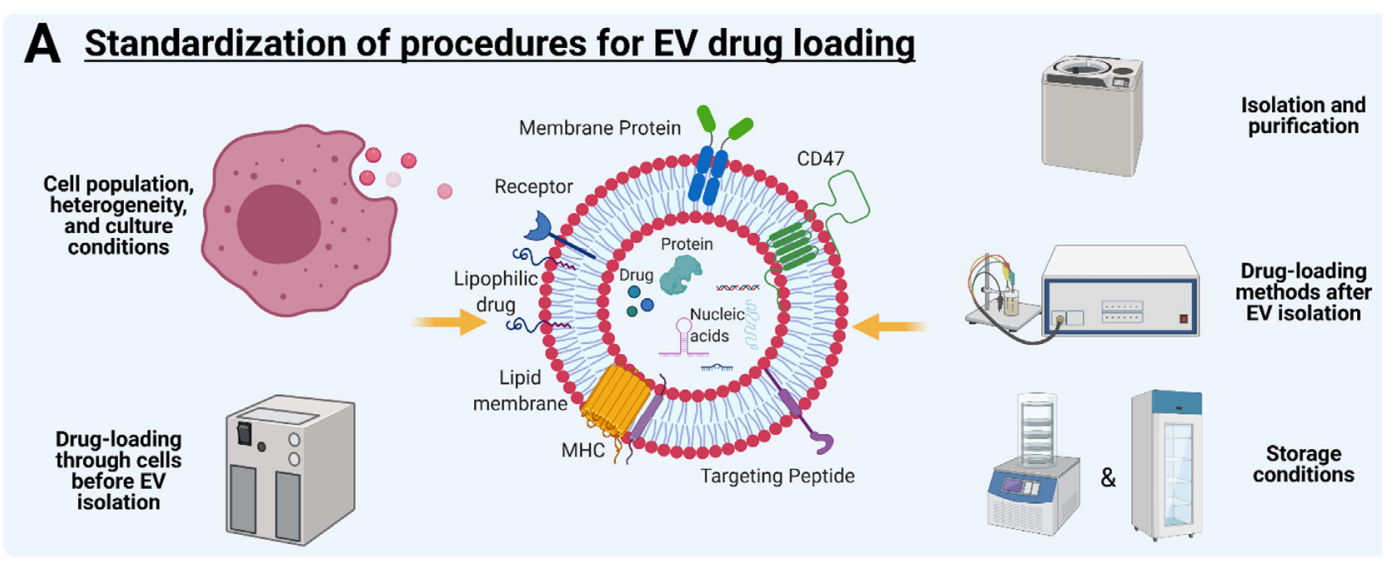

\section{B EVs as DDS towards clinical trials}

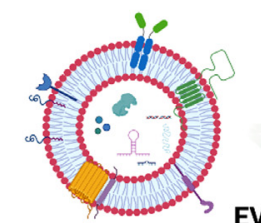

vS.

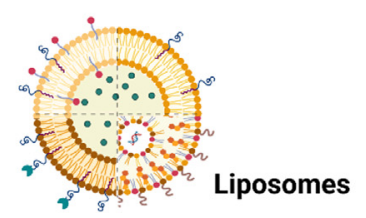

Processing

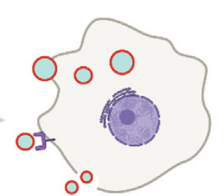

In vitro

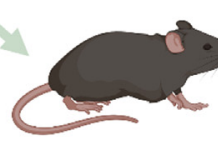

In vivo
Cellular uptake Intracellular trafficking Drug loading efficiency Stability

Administration methods

Biodistribution Drug trafficking efficiency Targeting

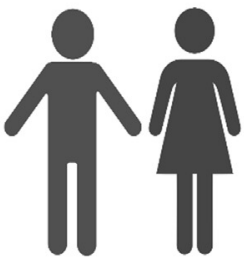

Clinical trials

Therapeutic effect

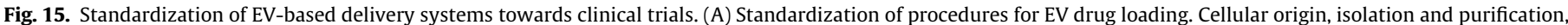

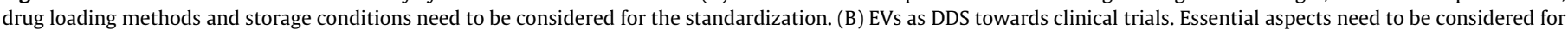
developing EV-based DDS at all stages, including in vitro screening, preclinical testing and clinical trials. Liposomes may be considered as a reference DDS.

Table 5

FDA/EMA guidelines point to a number of liposome characteristics that should be reported that are deemed important for liposomal product performance.

\begin{tabular}{|c|c|}
\hline Composition & $\begin{array}{l}\text { - Components of the liposome } \\
\text { - Quantities of the active substance and each lipid } \\
\text { - Molar ratio or percentage by weight of the lipid (including functional lipid) to the active substance }\end{array}$ \\
\hline Characterization & $\begin{array}{l}\text { - Particle size distribution } \\
\text { - Morphology and/or structure of the liposome } \\
\text { - Surface charge (zeta potential) } \\
\text { - Thermodynamic properties of the membrane } \\
\text { - Osmolality } \\
\text { - pH } \\
\text { - Aggregation } \\
\text { - Loading efficiency of the active substances } \\
\text { - Impurities } \\
\text { - Physical state of the encapsulated active substance } \\
\text { - Conformational structure, modification efficiency, and binding } \\
\text { - Capability }\end{array}$ \\
\hline In vitro release & $\begin{array}{l}\text { - Release profile of the active substance from the liposome } \\
\text { - For internal triggered release liposomes, release profile under conditions that reflect the } \\
\text { physiological environment } \\
\text { - For external triggered release liposome, release profile with external stimulation }\end{array}$ \\
\hline $\begin{array}{l}\text { Manufacturing process \& process controls of } \\
\text { liposome drug products }\end{array}$ & $\begin{array}{l}\text { - Process of formation of liposomes } \\
\text { - Encapsulation process of the active substance in liposomes } \\
\text { - Sizing process } \\
\text { - Process for surface modification } \\
\text { - Sterilization process } \\
\text { - Stability } \\
\text { - Endotoxin content } \\
\text { - Biological assay for efficacy }\end{array}$ \\
\hline
\end{tabular}

successful drug delivery across the BBB. Of notice, important progress has been made regarding the discovery of EVs which are capable of crossing specific biological barriers, such as the BBB in preclinical models. To illustrate this, exosomes derived from human neuronal stem cells and a brain endothelial cell line were able to cross the BBB and accumulate in injured sites [306] and 
Table 6

Hypothetical risk factor analysis of an EV product.

\begin{tabular}{|c|c|c|c|c|c|}
\hline & $\begin{array}{l}\text { Unwanted } \\
\text { immunogenicity }\end{array}$ & Treatment failure & $\begin{array}{l}\text { Disease } \\
\text { transmission }\end{array}$ & Tumor formation & Toxicity \\
\hline Cell starting material & $\begin{array}{l}\text { Possible HLA } \\
\text { mismatching }\end{array}$ & & $\begin{array}{l}\text { Information on cell } \\
\text { origin not complete }\end{array}$ & $\begin{array}{l}\text { Oncogenic transformation } \\
\text { because of cell source }\end{array}$ & \\
\hline Culture conditions & $\begin{array}{l}\text { Possible immune } \\
\text { reaction to animal } \\
\text { derived materials or } \\
\text { cells }\end{array}$ & $\begin{array}{l}\text { Composition compromises EV } \\
\text { activity - insufficient } \\
\text { production of bioactive } \\
\text { compounds }\end{array}$ & $\begin{array}{l}\text { Potential for } \\
\text { disease } \\
\text { transmission from } \\
\text { cell source, animal } \\
\text { derived materials }\end{array}$ & $\begin{array}{l}\text { Culture with growth factors or } \\
\text { hormones may induce tumor } \\
\text { formation }\end{array}$ & \\
\hline $\begin{array}{l}\text { Cell population, } \\
\text { heterogeneity \& } \\
\text { differentiation } \\
\text { potential }\end{array}$ & $\begin{array}{l}\text { Subpopulation } \\
\text { formation of cells } \\
\text { causes } \\
\text { immunogenicity }\end{array}$ & $\begin{array}{l}\text { Subpopulation formation } \\
\text { produces EVs with unfit } \\
\text { properties }\end{array}$ & & $\begin{array}{l}\text { Subpopulation formation of cells } \\
\text { produces EVs with higher } \\
\text { potential of oncogenic } \\
\text { transformation }\end{array}$ & \\
\hline $\begin{array}{l}\text { Genetic stability of } \\
\text { source cells }\end{array}$ & $\begin{array}{l}\text { Genetic instability } \\
\text { may increase } \\
\text { immunogenicity }\end{array}$ & $\begin{array}{l}\text { Genetic instability may result } \\
\text { in potential loss of secreted } \\
\text { bioactive substances }\end{array}$ & & & \\
\hline $\begin{array}{l}\text { Isolation and } \\
\text { Purification (IaP) }\end{array}$ & $\begin{array}{l}\text { Increased } \\
\text { immunogenicity } \\
\text { through IaP } \\
\text { procedures }\end{array}$ & $\begin{array}{l}\text { Potential impact of IaP } \\
\text { procedures on biological } \\
\text { activity }\end{array}$ & & & $\begin{array}{l}\text { Potential impact of IaP } \\
\text { procedures on toxicity }\end{array}$ \\
\hline Storage conditions & & $\begin{array}{l}\text { EVs lose their intrinsic activity } \\
\text { and vesicle stability (e.g. drug } \\
\text { leaking) }\end{array}$ & & & $\begin{array}{l}\text { Preservatives or } \\
\text { cryoprotective } \\
\text { reagents may confer } \\
\text { cytotoxicity }\end{array}$ \\
\hline Biodistribution & $\begin{array}{l}\text { Distribution to } \\
\text { different organs/cells } \\
\text { may increase risk of } \\
\text { immunogenicity }\end{array}$ & $\begin{array}{l}\text { Potential loss of activity due to } \\
\text { limited arrival at target site of } \\
\text { EVs }\end{array}$ & & $\begin{array}{l}\text { Tumor formation in different } \\
\text { organs }\end{array}$ & $\begin{array}{l}\text { Delivery of bioactive } \\
\text { substances in } \\
\text { unintended } \\
\text { microenvironments } \\
\text { may cause toxicity }\end{array}$ \\
\hline $\begin{array}{l}\text { Relevance of the } \\
\text { animal model }\end{array}$ & & $\begin{array}{l}\text { Available animal model is not } \\
\text { reflecting human disease }\end{array}$ & & $\begin{array}{l}\text { Age, dosing, immuno- } \\
\text { competence and duration of } \\
\text { animal study not appropriate for } \\
\text { detection of tumor formation; } \\
\text { tumorigenicity }\end{array}$ & \\
\hline Patient-related & $\begin{array}{l}\text { Risk for unwanted } \\
\text { immunogenicity due } \\
\text { to patient history }\end{array}$ & $\begin{array}{l}\text { Risk for treatment failure due } \\
\text { to patient history (age, } \\
\text { suboptimal microenvironment } \\
\text { and insufficient dose finding } \\
\text { data) }\end{array}$ & & $\begin{array}{l}\text { Risk for unwanted tissue } \\
\text { formation due to } \\
\text { microenvironment }\end{array}$ & \\
\hline Disease-related & & $\begin{array}{l}\text { Risk for suboptimal EV } \\
\text { performance due to target } \\
\text { tissue microenvironment }\end{array}$ & & & \\
\hline $\begin{array}{l}\text { Medical procedure- } \\
\text { related }\end{array}$ & $\begin{array}{l}\text { Unwanted immune } \\
\text { reaction \& allergy to } \\
\text { concomitant } \\
\text { substances at site of } \\
\text { application }\end{array}$ & & $\begin{array}{l}\text { Risk for infection } \\
\text { due to application } \\
\text { procedure }\end{array}$ & $\begin{array}{l}\text { Hypertrophic growth due to } \\
\text { application procedure }\end{array}$ & \\
\hline
\end{tabular}

brain cancer [66], respectively. Furthermore, EVs administered intranasally were shown effective for the delivery of curcumin to the brain, suggesting an alternative route for the treatment of brain inflammatory diseases [193].

\subsubsection{Emerging EV platforms}

Recent theranostic platforms that allow imaging of therapeutic EVs harbor great potential to get a more precise understanding of their ways of action. Magnetic EVs, through loading of iron oxide nanoparticles, represent a sensitive and specific way of isolating EVs, enhancing tumor targeting under a magnetic field, and tracking systemically administered therapeutic EVs through magnetic particle imaging [109,138,307]. Another emerging strategy is the direct transfer of mitochondria [308-310] through EVs, which was recently reported to improve post-infarct cardiac function in vivo by restoring intracellular bioenergetics and mitochondrial biogenesis in the recipient cardiomyocytes [310]. It is yet to be proven whether EVs are capable of efficiently transferring other organelles of therapeutic relevance. Additionally, improving other drug delivery nanoparticles by encapsulating or hybridizing them with EVs may also be attractive to those nanoparticles of therapeutic potential but with a fast metabolism, limited bioavailability and rapid clearance after systemic administration [196].

\subsection{Defining the niches of EVs in comparison to liposomes}

These important landmarks point to certain appealing characteristics of EVs as advanced delivery systems. It may be argued that their structural and functional complexity together with their innate therapeutic activity make EVs especially suited for multifactorial diseases/conditions (Table 4). Still, from a realistic perspective, EVs have a long way to go to improve many aspects such as drug loading, retention of drug, pharmaceutical stability during storage, PK, biodistribution and cGMP of clinical batches.

\subsection{Reaching the clinic: guidance on how to evaluate EVs as drug delivery systems}

The lack of standardization of reproducible procedures for EVs as DDS in the field is putting serious brakes on the clinical development of EVs (Fig. 15a,b). As a result, regulatory agencies like the FDA and EMA are considering risks and release-criteria for EV products [288], influenced to a certain extent by the guidance on liposomal products. Liposomes are generally composed of a handful of chemically defined molecules and produced under specified conditions, and there is a high level of control over the characteristics of the formulation. The FDA/EMA guidance on liposomal 


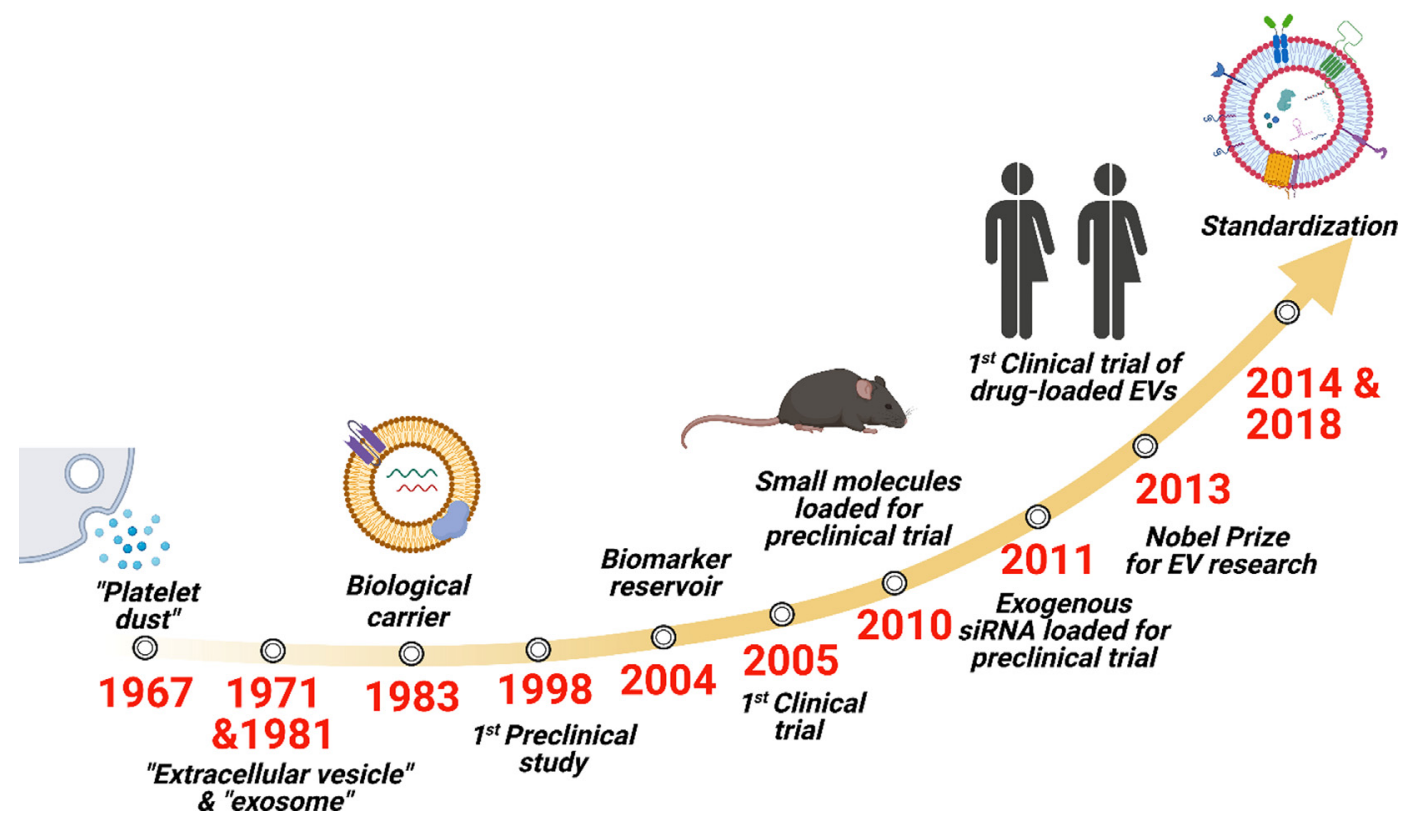

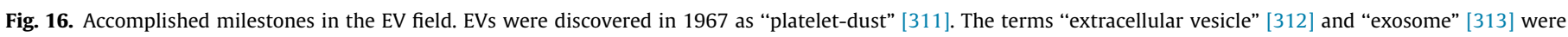

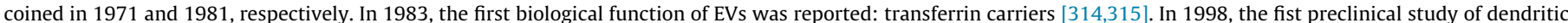

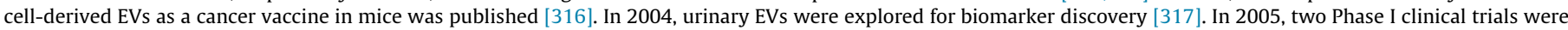

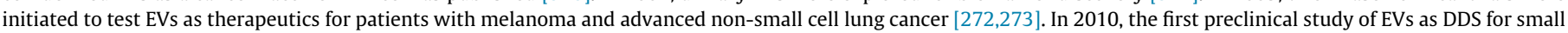

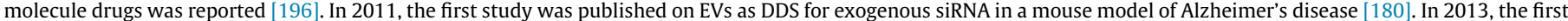

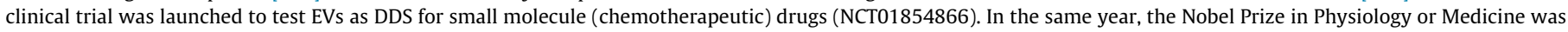

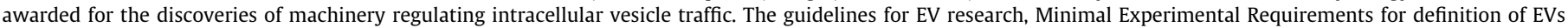
(MISEVs), were proposed in 2014 and revised in 2018 [8,53].

products, for example, provides clear specifications of the data that should be reported in the dossier on liposomal products (Table 5).

It is clear that the same level of specifications cannot be met by EVs as they are complex vesicles that come from living cells. As a result, the guidance for Advanced Therapy Medicinal Products (ATMP) seems to be a better fit, although the requirements in this guidance are less well defined due to the large variety of products and applications in this group of therapeutics. The ATMP guidelines are based on a risk stratification approach that consists of 4 steps:

1. To identify risks associated with the clinical use of the ATMP

2. To identify product specific risk factors contributing to each identified risk

3. To map the relevant data for each identified risk factor against each of the identified risk

4. To conclude on the risk factor - risk relationships

These analyses are used to fill a risk-risk factor table in which these are described. Table 6 provides a hypothetical analysis for an EV product.

\section{Conclusions and future perspectives}

EVs have been studied for over half a century and the field has been evolving quickly over the recent years, from the discovery to the applications in diagnostics, from preclinical studies to clinical trials on EVs as therapeutics and DDS, and to the recent standardization (i.e. guidelines) for EV research (Fig. 16).

The convergence of standardized procedures and regulations, together with the advent of new discoveries in more adequate EV donor cell types and drug loading procedures that do not compromise essential structural features of EVs, could possibly lead the field to future successes in the clinic. Furthermore, it is important to compare EVs with other delivery systems such as liposomes, and possibly to cellular systems such as therapeutic stem cells, to determine the 'fit-for-purpose'. In cancer treatment, for example, the primary objective is to deliver a maximal dose of chemotherapeutics to the tumor, which seems to be a closer fit with DDS like liposomes. On the other hand, EVs hold promise as DDS for their "homing" property, beyond the EPR effect, to specific tumors, their potential to cross certain biological barriers such as the BBB, their unique capacity to encapsulate certain biomolecules such as membrane receptors that are difficult to load into other DDS, and their plasticity for bioengineering. In fields like regenerative medicine that involve multifactorial biological processes, a complex natural delivery system like EVs can offer a competitive advantage. From this systematic review we conclude that there is an increasing use of EVs for drug delivery in cancer treatment, along with an extensive application of nucleic acids and small molecule drugs as APIs. We also notice an increasing tendency in the use of intravenous administration of EVs, accompanied with coating EVs with agents that result in increased target capacity (e.g. tumor antigens) and/or circulation time (e.g. PEG, CD47). We envision that the niches of EVs as DDS in the near future may be dominated by incorporation of membrane proteins and perhaps delivery of RNAs when the loading hurdles are solved, as well as delivery of proteins/peptides, for regenerative medicine and development of vaccines. There is a vast array of fields that may benefit from the use of EVs as DDS, but identification of the proper niches will come along with the maturation of the EV drug delivery field and a deeper comprehension of the intracellular mechanisms underlying EV uptake by and endosomal escape of the loaded compounds in target cells.

\section{Declaration of Competing Interest}

The authors declare that they have no known competing financial interests or personal relationships that could have appeared to influence the work reported in this paper. 


\section{Acknowledgments}

The authors would like to thank the funding support from the Singapore Ministry of Health's National Medical Research Council (NMRC/OFYIRG/0081/2018 to J.W.W., G.P. and G.S.), NUHS (NUHSRO/2018/095/RO5 + 5/Seed-Nov/05 to J.W.W.; NUHSRO/ 2021/034/TRP/09/Nanomedicine to X.C., G.S., and J.W.W.), NUS ODPRT Cross-Faculty Research Fund (CFGFY20P14 to J.W.W.), the National University of Singapore NanoNASH Program (NUHSRO/2020/002/NanoNash/LOA to G.S., G.P., and J.W.W.), the National University of Singapore start-up fund (NUHSRO/ 2020/133/Startup/08 to X.C.; NUHSRO/2019/077/STARTUP/03-OD PRT and NUHSRO/2019/077/STARTUP/03-NUSMed to G.S.), the National University of Singapore (R-148-000-296-114 and R-148000-284-114 to G.P.) and the RIE2020 Advanced Manufacturing and Engineering (AME) Industry Alignment Fund - Pre Positioning (IAF-PP) grant (A20G1a0046 and R-148-000-307-305 to G.P.), and the European Union's Horizon 2020 research and innovation programme under the Marie Skłodowska-Curie grant agreement (proEVLifeCycle, grant No 860303; and the B-SMART project, grant No 721058; to R.M.S). Figs. 1, 2, 14-16 were created with BioRender.com.

\section{Appendix A. Supplementary material}

Supplementary data to this article can be found online at https://doi.org/10.1016/j.addr.2021.05.011.

\section{References}

[1] O.P.B. Wiklander, M. Brennan, J. Lötvall, X.O. Breakefield, S.E.L. Andaloussi, Advances in therapeutic applications of extracellular vesicles, Sci. Transl. Med. 11 (2019) 1-16, https://doi.org/10.1126/scitranslmed.aav8521.

[2] S.Y. Chong, C.K. Lee, C. Huang, Y.H. Ou, C.J. Charles, A.M. Richards, Y.R. Neupane, M.V. Pavon, O. Zharkova, G. Pastorin, J.W. Wang, Extracellular vesicles in cardiovascular diseases: alternative biomarker sources, therapeutic agents, and drug delivery carriers, Int. J. Mol. Sci. 20 (2019), https://doi.org/10.3390/ijms20133272.

[3] O.G. de Jong, S.A.A. Kooijmans, D.E. Murphy, L. Jiang, M.J.W. Evers, J.P.G. Sluijter, P. Vader, R.M. Schiffelers, Drug delivery with extracellular vesicles: from imagination to innovation, Acc. Chem. Res. 52 (2019) 1761-1770, https://doi.org/10.1021/acs.accounts.9b00109.

[4] E. Woith, G. Fuhrmann, M.F. Melzig, Extracellular vesicles-connecting kingdoms, Int. J. Mol. Sci. 20 (2019) 5695, https://doi.org/10.3390/ ijms20225695.

[5] R. Kalluri, V.S. LeBleu, The biology, function, and biomedical applications of exosomes, Science 367 (2020), https://doi.org/10.1126/science.aau6977.

[6] M.A. Mori, R.G. Ludwig, R. Garcia-Martin, B.B. Brandão, C.R. Kahn, Extracellular miRNAs: from biomarkers to mediators of physiology and disease, Cell Metab. 30 (2019) 656-673, https://doi.org/10.1016/j. cmet.2019.07.011.

[7] L. Tong, H. Hao, X. Zhang, Z. Zhang, Y. Lv, L. Zhang, H. Yi, Oral administration of bovine milk-derived extracellular vesicles alters the gut microbiota and enhances intestinal immunity in mice, Mol. Nutr. Food Res. 64 (2020), https:// doi.org/10.1002/mnfr.201901251 e1901251.

[8] C. Thery, K.W. Witwer, E. Aikawa, M.J. Alcaraz, J.D. Anderson, R. Andriantsitohaina, A. Antoniou, T. Arab, F. Archer, G.K. Atkin-Smith, D.C. Ayre, J.M. Bach, D. Bachurski, H. Baharvand, L. Balaj, S. Baldacchino, N.N. Bauer, A.A. Baxter, M. Bebawy, C. Beckham, A. Bedina Zavec, A. Benmoussa, A. C. Berardi, P. Bergese, E. Bielska, C. Blenkiron, S. Bobis-Wozowicz, E. Boilard, W. Boireau, A. Bongiovanni, F.E. Borras, S. Bosch, C.M. Boulanger, X. Breakefield, A.M. Breglio, M.A. Brennan, D.R. Brigstock, A. Brisson, M.L. Broekman, J.F. Bromberg, P. Bryl-Gorecka, S. Buch, A.H. Buck, D. Burger, S. Busatto, D. Buschmann, B. Bussolati, E.I. Buzas, J.B. Byrd, G. Camussi, D.R. Carter, S. Caruso, L.W. Chamley, Y.T. Chang, C. Chen, S. Chen, L. Cheng, A.R. Chin, A. Clayton, S.P. Clerici, A. Cocks, E. Cocucci, R.J. Coffey, A. Cordeiro-daSilva, Y. Couch, F.A. Coumans, B. Coyle, R. Crescitelli, M.F. Criado, C. D'SouzaSchorey, S. Das, A. Datta Chaudhuri, P. de Candia, E.F. De Santana, O. De Wever, H.A. Del Portillo, T. Demaret, S. Deville, A. Devitt, B. Dhondt, D. Di Vizio, L.C. Dieterich, V. Dolo, A.P. Dominguez Rubio, M. Dominici, M.R. Dourado, T.A. Driedonks, F.V. Duarte, H.M. Duncan, R.M. Eichenberger, K. Ekstrom, S. El Andaloussi, C. Elie-Caille, U. Erdbrugger, J.M. Falcon-Perez, F. Fatima, J.E. Fish, M. Flores-Bellver, A. Forsonits, A. Frelet-Barrand, F. Fricke, G. Fuhrmann, S. Gabrielsson, A. Gamez-Valero, C. Gardiner, K. Gartner, R. Gaudin, Y.S. Gho, B. Giebel, C. Gilbert, M. Gimona, I. Giusti, D.C. Goberdhan, A. Gorgens, S.M. Gorski, D.W. Greening, J.C. Gross, A. Gualerzi, G.N. Gupta, D. Gustafson, A.
Handberg, R.A. Haraszti, P. Harrison, H. Hegyesi, A. Hendrix, A.F. Hill, F.H. Hochberg, K.F. Hoffmann, B. Holder, H. Holthofer, B. Hosseinkhani, G. Hu, Y Huang, V. Huber, S. Hunt, A.G. Ibrahim, T. Ikezu, J.M. Inal, M. Isin, A. Ivanova, H.K. Jackson, S. Jacobsen, S.M. Jay, M. Jayachandran, G. Jenster, L. Jiang, S.M. Johnson, J.C. Jones, A. Jong, T. Jovanovic-Talisman, S. Jung, R. Kalluri, S.I Kano, S. Kaur, Y. Kawamura, E.T. Keller, D. Khamari, E. Khomyakova, A. Khvorova, P. Kierulf, K.P. Kim, T. Kislinger, M. Klingeborn, D.J. Klinke 2nd, M. Kornek, M.M. Kosanovic, A.F. Kovacs, E.M. Kramer-Albers, S. Krasemann, M. Krause, I.V. Kurochkin, G.D. Kusuma, S. Kuypers, S. Laitinen, S.M. Langevin, L. R. Languino, J. Lannigan, C. Lasser, L.C. Laurent, G. Lavieu, E. Lazaro-Ibanez, S. Le Lay, M.S. Lee, Y.X.F. Lee, D.S. Lemos, M. Lenassi, A. Leszczynska, I.T. Li, K. Liao, S.F. Libregts, E. Ligeti, R. Lim, S.K. Lim, A. Line, K. Linnemannstons, A. Llorente, C.A. Lombard, M.J. Lorenowicz, A.M. Lorincz, J. Lotvall, J. Lovett, M. C. Lowry, X. Loyer, Q. Lu, B. Lukomska, T.R. Lunavat, S.L. Maas, H. Malhi, A. Marcilla, J. Mariani, J. Mariscal, E.S. Martens-Uzunova, L. Martin-Jaular, M.C. Martinez, V.R. Martins, M. Mathieu, S. Mathivanan, M. Maugeri, L.K. McGinnis, M.J. McVey, D.G. Meckes Jr., K.L. Meehan, I. Mertens, V.R. Minciacchi, A. Moller, E.N. Nolte-'t Hoen, N. Noren Hooten, L. O’Driscoll, T. O'Grady, A. O'Loghlen, T. Ochiya, M. Olivier, A. Ortiz, L.A. Ortiz, X Osteikoetxea, O. Ostergaard, M. Ostrowski, J. Park, D.M. Pegtel, H. Peinado, F. Perut, M.W. Pfaffl, D.G. Phinney, B.C. Pieters, R.C. Pink, D.S. Pisetsky, E. Pogge von Strandmann, I. Polakovicova, I.K. Poon, B.H. Powell, I. Prada, L. Pulliam, P. Quesenberry, A. Radeghieri, R.L. Raffai, S. Raimondo, J. Rak, M.I. Ramirez, G. Raposo, M.S. Rayyan, N. Regev-Rudzki, F.L. Ricklefs, P.D. Robbins, D.D. Roberts, S.C. Rodrigues, E. Rohde, S. Rome, K.M. Rouschop, A. Rughetti, A.E. Russell, P. Saa, S. Sahoo, E. Salas-Huenuleo, C. Sanchez, J.A. Saugstad, M. J. Saul, R.M. Schiffelers, R. Schneider, T.H. Schoyen, A. Scott, E. Shahaj, S. Sharma, O. Shatnyeva, F. Shekari, G.V. Shelke, A.K. Shetty, K. Shiba, P.R. Siljander, A.M. Silva, A. Skowronek, O.L. Snyder 2nd, R.P. Soares, B.W. Sodar, C. Soekmadji, J. Sotillo, P.D. Stahl, W. Stoorvogel, S.L. Stott, E.F. Strasser, S. Swift, H. Tahara, M. Tewari, K. Timms, S. Tiwari, R. Tixeira, M. Tkach, W.S. Toh, R. Tomasini, A.C. Torrecilhas, J.P. Tosar, V. Toxavidis, L. Urbanelli, P. Vader, B.W. van Balkom, S.G. van der Grein, J. Van Deun, M.J. van Herwijnen, K. Van Keuren-Jensen, G. van Niel, M.E. van Royen, A.J. van Wijnen, M.H. Vasconcelos, I.J. Vechetti Jr., T.D. Veit, L.J. Vella, E. Velot, F.J. Verweij, B. Vestad, J.L. Vinas, T. Visnovitz, K.V. Vukman, J. Wahlgren, D.C. Watson, M.H. Wauben, A. Weaver, J.P. Webber, V. Weber, A.M. Wehman, D.J. Weiss, J.A. Welsh, S. Wendt, A.M. Wheelock, Z. Wiener, L. Witte, J. Wolfram, A. Xagorari, P. Xander, J. Xu, X. Yan, M. Yanez-Mo, H. Yin, Y. Yuana, V. Zappulli, J. Zarubova, V. Zekas, J.Y. Zhang, Z. Zhao, L. Zheng, A.R. Zheutlin, A.M. Zickler P. Zimmermann, A.M. Zivkovic, D. Zocco, E.K. Zuba-Surma, Minimal information for studies of extracellular vesicles 2018 (MISEV2018): a position statement of the International Society for Extracellular Vesicles and update of the MISEV2014 guidelines, J. Extracell. Vesicles. 7 (2018) (2018) 1535750, https://doi.org/10.1080/20013078.2018.1535750.

[9] B. Gyorgy, T.G. Szabo, M. Pasztoi, Z. Pal, P. Misjak, B. Aradi, V. Laszlo, E. Pallinger, E. Pap, A. Kittel, G. Nagy, A. Falus, E.I. Buzas, Membrane vesicles, current state-of-the-art: emerging role of extracellular vesicles, Cell Mol. Life Sci. 68 (2011) 2667-2688, https://doi.org/10.1007/s00018-011-0689-3.

[10] S. El Andaloussi, I. Mäger, X.O. Breakefield, M.J.A. Wood, Extracellular vesicles: biology and emerging therapeutic opportunities, Nat. Rev. Drug Discov. 12 (2013) 347-357, https://doi.org/10.1038/nrd3978.

[11] E. Cocucci, J. Meldolesi, Ectosomes and exosomes: shedding the confusion between extracellular vesicles, Trends. Cell. Biol. 25 (2015) 364-372, https:// doi.org/10.1016/j.tcb.2015.01.004.

[12] E. Willms, C. Cabañas, I. Mäger, M.J.A. Wood, P. Vader, Extracellular vesicle heterogeneity: subpopulations, isolation techniques, and diverse functions in cancer progression, Front. Immunol. 9 (2018), https://doi.org/ 10.3389/fimmu.2018.00738.

[13] L. Console, M. Scalise, C. Indiveri, Exosomes in inflammation and role as biomarkers, Clin. Chim. Acta. 488 (2019) 165-171, https://doi.org/10.1016/j. cca.2018.11.009.

[14] F.M. Barros, F. Carneiro, J.C. Machado, S.A. Melo, Exosomes and immune response in cancer: friends or foes?, Front Immunol. 9 (2018), https://doi.org/ 10.3389/fimmu.2018.00730.

[15] J. Kowal, G. Arras, M. Colombo, M. Jouve, J.P. Morath, B. Primdal-Bengtson, F. Dingli, D. Loew, M. Tkach, C. Théry, Proteomic comparison defines novel markers to characterize heterogeneous populations of extracellular vesicle subtypes, Proc. Natl. Acad. Sci. U S A. 113 (2016) E968-E977, https://doi.org/ 10.1073/pnas.1521230113.

[16] S.J. Gould, G. Raposo, As we wait: coping with an imperfect nomenclature for extracellular vesicles, J. Extracell. Vesicles. 2 (2013), https://doi.org/ 10.3402/jev.v2i0.20389.

[17] G. van Niel, G. D'Angelo, G. Raposo, Shedding light on the cell biology of extracellular vesicles, Nat. Rev. Mol. Cell. Biol. 19 (2018) 213-228, https://doi. org/10.1038/nrm.2017.125.

[18] O.M. Elsharkasy, J.Z. Nordin, D.W. Hagey, O.G. de Jong, R.M. Schiffelers, S.E.L. Andaloussi, P. Vader, Extracellular vesicles as drug delivery systems: why and how?, Adv Drug. Deliv. Rev. 159 (2020) 332-343, https://doi.org/10.1016/j. addr.2020.04.004.

[19] Y. Yuana, A. Sturk, R. Nieuwland, Extracellular vesicles in physiological and pathological conditions, Blood Rev. 27 (2013) 31-39, https://doi.org/10.1016/ j.blre.2012.12.002.

[20] E. D'Asti, S. Chennakrishnaiah, T.H. Lee, J. Rak, Extracellular vesicles in brain tumor progression, Cell Mol. Neurobiol. 36 (2016) 383-407, https://doi.org/ 10.1007/s10571-015-0296-1. 
[21] J. Kong, H. Tian, F. Zhang, Z. Zhang, J. Li, X. Liu, X. Li, J. Liu, X. Li, D. Jin, X. Yang, B. Sun, T. Guo, Y. Luo, Y. Lu, B. Lin, T. Liu, Extracellular vesicles of carcinomaassociated fibroblasts creates a pre-metastatic niche in the lung through activating fibroblasts, Mol. Cancer. 18 (2019) 175, https://doi.org/10.1186/ s12943-019-1101-4.

[22] C. Federici, F. Petrucci, S. Caimi, A. Cesolini, M. Logozzi, M. Borghi, S. D’llio, L. Lugini, N. Violante, T. Azzarito, C. Majorani, D. Brambilla, S. Fais, Exosome release and low $\mathrm{pH}$ belong to a framework of resistance of human melanoma cells to cisplatin, PLoS One. 9 (2014), https://doi.org/10.1371/journal. pone.0088193 e88193.

[23] A.T.A. Halim, N.A.F.M. Ariffin, M. Azlan, Review: the multiple roles of monocytic microparticles, Inflammation. 39 (2016) 1277-1284, https://doi. org/10.1007/s10753-016-0381-8.

[24] M. Yanez-Mo, P.R. Siljander, Z. Andreu, A.B. Zavec, F.E. Borras, E.I. Buzas, K. Buzas, E. Casal, F. Cappello, J. Carvalho, E. Colas, A. Cordeiro-da Silva, S. Fais, J. M. Falcon-Perez, I.M. Ghobrial, B. Giebel, M. Gimona, M. Graner, I. Gursel, M. Gursel, N.H. Heegaard, A. Hendrix, P. Kierulf, K. Kokubun, M. Kosanovic, V. Kralj-Iglic, E.M. Kramer-Albers, S. Laitinen, C. Lasser, T. Lener, E. Ligeti, A. Line, G. Lipps, A. Llorente, J. Lotvall, M. Mancek-Keber, A. Marcilla, M. Mittelbrunn, I. Nazarenko, E.N. Nolte-t' Hoen, T.A. Nyman, L. O’Driscoll, M. Olivan, C. Oliveira, E. Pallinger, H.A. Del Portillo, J. Reventos, M. Rigau, E. Rohde, M. Sammar, F. Sanchez-Madrid, N. Santarem, K. Schallmoser, M.S. Ostenfeld, W. Stoorvogel, R. Stukelj, S.G. Van der Grein, M.H. Vasconcelos, M.H. Wauben, O. De Wever, Biological properties of extracellular vesicles and their physiological functions, J. Extracell. Vesicles. 4 (2015) 27066, http://doi.org/ 10.3402/jev.v4.27066.

[25] X. Wang, W. Huang, G. Liu, W. Cai, R.W. Millard, Y. Wang, J. Chang, T. Peng, G.-C. Fan, Cardiomyocytes mediate anti-angiogenesis in type 2 diabetic rats through the exosomal transfer of miR-320 into endothelial cells, J. Mol. Cell. Cardiol. 74 (2014) 139-150, https://doi.org/10.1016/j.yjmcc. 2014.05.001.

[26] G. Lombardo, P. Dentelli, G. Togliatto, A. Rosso, M. Gili, S. Gallo, M.C. Deregibus, G. Camussi, M.F. Brizzi, Activated Stat5 trafficking via endothelial cell-derived extracellular vesicles controls IL-3 pro-angiogenic paracrine action, Sci. Rep. 6 (2016) 1-14, https://doi.org/10.1038/srep25689.

[27] M.T. Roefs, J.P.G. Sluijter, P. Vader, Extracellular vesicle-associated proteins in tissue repair, Trends Cell Biol. 30 (2020) 990-1013, https://doi.org/10.1016/j. tcb.2020.09.009.

[28] C. Hromada, S. Mühleder, J. Grillari, H. Redl, W. Holnthoner, Endothelial extracellular vesicles-promises and challenges, Front. Physiol. 8 (2017), https://doi.org/10.3389/fphys.2017.00275.

[29] M.L. Liu, M.P. Reilly, P. Casasanto, S.E. McKenzie, K.J. Williams, Cholesterol enrichment of human monocyte/macrophages induces surface exposure of phosphatidylserine and the release of biologically-active tissue factorpositive microvesicles, Arterioscler. Thromb. Vasc. Biol. 27 (2007) 430-435, https://doi.org/10.1161/01.ATV.0000254674.47693.e8.

[30] L.C.P. Azevedo, M. Janiszewski, V. Pontieri, M.d.A. Pedro, E. Bassi, P.J.F. Tucci, F. R.M. Laurindo, Platelet-derived exosomes from septic shock patients induce myocardial dysfunction, Crit. Care. 11 (2007) 1-10, https://doi.org/10.1186/ cc6176.

[31] J.D. Hutcheson, E. Aikawa, Extracellular vesicles in cardiovascular homeostasis and disease, Curr. Opin. Cardiol. 33 (2018) 290-297, https:// doi.org/10.1097/HCO.0000000000000510.

[32] A. Hafiane, S.S. Daskalopoulou, Extracellular vesicles characteristics and emerging roles in atherosclerotic cardiovascular disease, Metabolism. 85 (2018) 213-222, https://doi.org/10.1016/j.metabol.2018.04.008.

[33] X. Zhou, F. Xie, L. Wang, L. Zhang, S. Zhang, M. Fang, F. Zhou, The function and clinical application of extracellular vesicles in innate immune regulation, Cell Mol. Immunol. 17 (2020) 323-334, https://doi.org/10.1038/s41423-0200391-1.

[34] R.E. Lane, D. Korbie, M.M. Hill, M. Trau, Extracellular vesicles as circulating cancer biomarkers: opportunities and challenges, Clin. Transl. Med. 7 (2018), https://doi.org/10.1186/s40169-018-0192-7.

[35] S. Pan, Y. Zhang, A. Natalia, C.Z.J. Lim, N.R.Y. Ho, B. Chowbay, T.P. Loh, J.K.C. Tam, H. Shao, Extracellular vesicle drug occupancy enables real-time monitoring of targeted cancer therapy, Nat. Nanotechnol. (2021), https:// doi.org/10.1038/s41565-021-00872-W.

[36] O.G. De Jong, B.W.M. Van Balkom, R.M. Schiffelers, C.V.C. Bouten, M.C. Verhaar, Extracellular vesicles: potential roles in regenerative medicine, Front. Immunol. 5 (2014), https://doi.org/10.3389/fimmu.2014.00608.

[37] M. Romano, A. Zendrini, L. Paolini, S. Busatto, A.C. Berardi, P. Bergese, A. Radeghieri, Extracellular vesicles in regenerative medicine, Elsevier (2020) 29-58, https://doi.org/10.1016/B978-0-12-817838-6.00002-4.

[38] N.S. Roy, C. Cleren, S.K. Singh, L. Yang, M.F. Beal, S.A. Goldman, Functional engraftment of human ES cell-derived dopaminergic neurons enriched by coculture with telomerase-immortalized midbrain astrocytes, Nat. Med. 12 (2006) 1259-1268, https://doi.org/10.1038/nm1495.

[39] H. Ning, F. Yang, M. Jiang, L. Hu, K. Feng, J. Zhang, Z. Yu, B. Li, C. Xu, Y. Li, J. Wang, J. Hu, X. Lou, H. Chen, The correlation between cotransplantation of mesenchymal stem cells and higher recurrence rate in hematologic malignancy patients: outcome of a pilot clinical study, Leukemia. 22 (2008) 593-599, https://doi.org/10.1038/sj.leu.2405090.

[40] J.-O. Jeong, J.W. Han, J.-M. Kim, H.-J. Cho, C. Park, N. Lee, D.-W. Kim, Y.-S. Yoon, Malignant tumor formation after transplantation of short-term cultured bone marrow mesenchymal stem cells in experimental myocardial infarction and diabetic neuropathy, Circ. Res. 108 (2011) 1340-1347, https://doi.org/ 10.1161/CIRCRESAHA.110.239848.

[41] F. Erdö, C. Bührle, J. Blunk, M. Hoehn, Y. Xia, B. Fleischmann, M. Föcking, E. Küstermann, E. Kolossov, J. Hescheler, K.-A. Hossmann, T. Trapp, Hostdependent tumorigenesis of embryonic stem cell transplantation in experimental stroke, J. Cereb. Blood Flow Metab. 23 (2003) 780-785, https://doi.org/10.1097/01.WCB.0000071886.63724.FB.

[42] B.J. Dlouhy, O. Awe, R.C. Rao, P.A. Kirby, P.W. Hitchon, Autograft-derived spinal cord mass following olfactory mucosal cell transplantation in a spinal cord injury patient, J. Neurosurg. Spine 21 (2014) 618-622, https://doi.org/ 10.3171/2014.5.SPINE13992.

[43] J.M. Vicencio, D.M. Yellon, V. Sivaraman, D. Das, C. Boi-Doku, S. Arjun, Y Zheng, J.A. Riquelme, J. Kearney, V. Sharma, G. Multhoff, A.R. Hall, S.M. Davidson, Plasma exosomes protect the myocardium from ischemiareperfusion injury, J. Am. Coll. Cardiol. 65 (2015) 1525-1536, https://doi. org/10.1016/j.jacc.2015.02.026.

[44] K. Kusuzaki, T. Matsubara, H. Murata, M. Logozzi, E. Iessi, R. Di Raimo, F. Carta, C.T. Supuran, S. Fais, Natural extracellular nanovesicles and photodynamic molecules: is there a future for drug delivery?, J Enzyme Inhib. Med. Chem. 32 (2017) 908-916, https://doi.org/10.1080/14756366.2017.1335310.

[45] K.B. Johnsen, J. Mar, M. Najbjerg, L. Pilgaard, T. Moos, M. Duroux, Biochimica et Biophysica Acta A comprehensive overview of exosomes as drug delivery vehicles - Endogenous nanocarriers for targeted cancer therapy, BBA. Rev. Cancer 2014 (1846) 75-87, https://doi.org/10.1016/j.bbcan.2014.04.005.

[46] B.A. Potz, L.A. Scrimgeour, V.I. Pavlov, N.R. Sodha, M. Ruhul Abid, F.W. Sellke, M.R. Abid, F.W. Sellke, M. Ruhul Abid, F.W. Sellke, Extracellular vesicle injection improves myocardial function and increases angiogenesis in a swine model of chronic ischemia, J. Am. Heart Assoc. 7 (2018), https://doi.org/ 10.1161/JAHA.117.008344.

[47] S. Montaner-Tarbes, E. Novell, V. Tarancón, F.E. Borrás, M. Montoya, L. Fraile, H.A. del Portillo, Targeted-pig trial on safety and immunogenicity of serumderived extracellular vesicles enriched fractions obtained from Porcine Respiratory and Reproductive virus infections, Sci. Rep. 8 (2018) 17487, https://doi.org/10.1038/s41598-018-36141-5.

[48] R. Vakili-Ghartavol, S.M. Rezayat, R. Faridi-Majidi, K. Sadri, M.R. Jaafari, Optimization of Docetaxel Loading Conditions in Liposomes: proposing potential products for metastatic breast carcinoma chemotherapy, Sci. Rep. 10 (2020) 1-14, https://doi.org/10.1038/s41598-020-62501-1.

[49] D.S. Sutaria, M. Badawi, M.A. Phelps, T.D. Schmittgen, Achieving the promise of therapeutic extracellular vesicles: the devil is in details of therapeutic loading, Pharm. Res. 34 (2017) 1053-1066, https://doi.org/10.1007/s11095017-2123-5.

[50] D. Moher, A. Liberati, J. Tetzlaff, D.G. Altman, Preferred reporting items for systematic reviews and meta-analyses: the PRISMA statement, Int. J. Surg. 8 (2010) 336-341, https://doi.org/10.1016/j.ijsu.2010.02.007.

[51] J.P.T. Higgins, J. Thomas, J. Chandler, M. Cumpston, T. Li, M.J. Page, V.A. Welch (Eds.), Cochrane Handbook for Systematic Reviews of Interventions version 6.2 (updated February 2021). Cochrane, 2021. Available from www.training.cochrane.org/handbook.

[52] K.W. Witwer, E.I. Buzás, L.T. Bemis, A. Bora, C. Lässer, J. Lötvall, E.N. Nolte-t' Hoen, M.G. Piper, S. Sivaraman, J. Skog, C. Théry, M.H. Wauben, F. Hochberg, Standardization of sample collection, isolation and analysis methods in extracellular vesicle research, J. Extracell. Vesicles. 2 (2013) 20360, https:// doi.org/10.3402/jev.v2i0.20360.

[53] J. Lotvall, A.F. Hill, F. Hochberg, E.I. Buzas, D. Di Vizio, C. Gardiner, Y.S. Gho, I.V. Kurochkin, S. Mathivanan, P. Quesenberry, S. Sahoo, H. Tahara, M.H. Wauben, K.W. Witwer, C. Thery, Minimal experimental requirements for definition of extracellular vesicles and their functions: a position statement from the International Society for Extracellular Vesicles, J. Extracell. Vesicles. 3 (2014) 26913, https://doi.org/10.3402/jev.v3.26913.

[54] C. Huang, Y.R. Neupane, X.C. Lim, R. Shekhani, B. Czarny, M.G. Wacker, G. Pastorin, J.W. Wang, Extracellular vesicles in cardiovascular disease, Elsevier Inc., Adv. Clin. Chem. 2020, in press, https://doi.org/10.1016/bs.acc.2020.08.006.

[55] S.W. Ferguson, J. Nguyen, Exosomes as therapeutics: the implications of molecular composition and exosomal heterogeneity, J. Control Release. 228 (2016) 179-190, https://doi.org/10.1016/j.jconrel.2016.02.037.

[56] G. Li, J. Wang, M. Xu, H. Zhang, C. Tu, J. Yang, X. Chen, Q. Yao, P. Lan, M. Xie, Engineered exosome for NIR-triggered drug delivery and superior synergistic chemo-phototherapy in a glioma model, Appl. Mater. Today. 20 (2020), https://doi.org/10.1016/j.apmt.2020.100723.

[57] Z. Yang, J. Shi, J. Xie, Y. Wang, J. Sun, T. Liu, Y. Zhao, X. Zhao, X. Wang, Y. Ma, V. Malkoc, C. Chiang, W. Deng, Y. Chen, Y. Fu, K.J. Kwak, Y. Fan, C. Kang, C. Yin, J. Rhee, P. Bertani, J. Otero, W. Lu, K. Yun, A.S. Lee, W. Jiang, L. Teng, B.Y.S. Kim, L. J. Lee, Large-scale generation of functional mRNA-encapsulating exosomes via cellular nanoporation, Nat. Biomed. Eng. 4 (2020) 69-83, https://doi.org/ 10.1038/s41551-019-0485-1.

[58] G. Jia, Y. Han, Y. An, Y. Ding, C. He, X. Wang, Q. Tang, NRP-1 targeted and cargo-loaded exosomes facilitate simultaneous imaging and therapy of glioma in vitro and in vivo, Biomaterials. 178 (2018) 302-316, https://doi. org/10.1016/j.biomaterials.2018.06.029.

[59] F.M.F. Lang, A. Hossain, J. Gumin, E.N. Momin, Y. Shimizu, D. Ledbetter, T. Shahar, S. Yamashita, B. Parker Kerrigan, J. Fueyo, R. Sawaya, F.M.F. Lang, Mesenchymal stem cells as natural biofactories for exosomes carrying miR124a in the treatment of gliomas, Neuro, Oncol. 20 (2018) 380-390, https:// doi.org/10.1093/neuonc/nox152. 
[60] G. Kim, M. Kim, Y. Lee, J. Woo, D. Won, M. Lee, Systemic delivery of microRNA-21 antisense oligonucleotides to the brain using T7-peptide decorated exosomes, J. Control Release. 317 (2020) 273-281, https://doi. org/10.1016/j.jconrel.2019.11.009.

[61] D. Gulei, I. Berindan-Neagoe, Activation of necroptosis by engineered self tumor-derived exosomes loaded with CRISPR/Cas9, Mol. Ther. Nucleic Acids. 17 (2019) 448-451, https://doi.org/10.1016/j.omtn.2019.05.032.

[62] Q. Zhu, X. Ling, Y. Yang, J. Zhang, Q. Li, X. Niu, G. Hu, B. Chen, H. Li, Y. Wang, Z. Deng, Embryonic stem cells-derived exosomes endowed with targeting properties as chemotherapeutics delivery vehicles for glioblastoma therapy, Adv. Sci. 6 (2019) 1801899, https://doi.org/10.1002/ advs.201801899.

[63] H. Monfared, Y. Jahangard, M. Nikkhah, J. Mirnajafi-Zadeh, S.J. Mowla, Potential therapeutic effects of exosomes packed with a miR-21-sponge construct in a rat model of glioblastoma, Front. Oncol. 9 (2019) 1-11, https:// doi.org/10.3389/fonc.2019.00782.

[64] E.P. Erkan, D. Senfter, S. Madlener, G. Jungwirth, T. Ströbel, N. Saydam, O. Saydam, Extracellular vesicle-mediated suicide mRNA/protein delivery inhibits glioblastoma tumor growth in vivo, Cancer Gene Ther. 24 (2017) 38-44, https://doi.org/10.1038/cgt.2016.78.

[65] T. Yang, B. Fogarty, B. LaForge, S. Aziz, T. Pham, L. Lai, S. Bai, Delivery of small interfering RNA to inhibit vascular endothelial growth factor in zebrafish using natural brain endothelia cell-secreted exosome nanovesicles for the treatment of brain cancer, AAPS. J. 19 (2017) 475-486, https://doi.org/ 10.1208/s12248-016-0015-y.

[66] T. Yang, P. Martin, B. Fogarty, A. Brown, K. Schurman, R. Phipps, V.P. Yin, P. Lockman, S. Bai, Exosome delivered anticancer drugs across the blood-brain barrier for brain cancer therapy in danio rerio, Pharm. Res. 32 (2015) 20032014, https://doi.org/10.1007/s11095-014-1593-y.

[67] Z. Ye, T. Zhang, W. He, H. Jin, C. Liu, Z. Yang, J. Ren, Methotrexate-loaded extracellular vesicles functionalized with therapeutic and targeted peptides for the treatment of glioblastoma multiforme, ACS. Appl. Mater. Interfaces. 10 (2018) 12341-12350, https://doi.org/10.1021/acsami.7b18135.

[68] M. Katakowski, B. Buller, X. Zheng, Y. Lu, T. Rogers, O. Osobamiro, W. Shu, F. Jiang, M. Chopp, Exosomes from marrow stromal cells expressing miR-146b inhibit glioma growth, Cancer Lett. 335 (2013) 201-204, https://doi.org/ 10.1016/j.canlet.2013.02.019.

[69] C. Feng, Z. Xiong, C. Wang, W. Xiao, H. Xiao, K. Xie, K. Chen, H. Liang, X. Zhang, H. Yang, Folic acid-modified Exosome-PH20 enhances the efficiency of therapy via modulation of the tumor microenvironment and directly inhibits tumor cell metastasis, Bioact. Mater. 6 (2021) 963-974, https://doi. org/10.1016/j.bioactmat.2020.09.014.

[70] X. Shi, Q. Cheng, T. Hou, M. Han, G. Smbatyan, J.E. Lang, A.L. Epstein, H.J. Lenz, Y. Zhang, Genetically engineered cell-derived nanoparticles for targeted breast cancer immunotherapy, Mol. Ther. 28 (2020) 536-547, https://doi.org/ 10.1016/j.ymthe.2019.11.020.

[71] M.J. Haney, Y. Zhao, Y.S. Jin, S.M. Li, J.R. Bago, N.L. Klyachko, A.V. Kabanov, E.V. Batrakova, Macrophage-derived extracellular vesicles as drug delivery systems for triple negative breast cancer (TNBC) therapy, J. Neuroimmune Pharmacol. 15 (2020) 487-500, https://doi.org/10.1007/s11481-019-098849.

[72] D. Hao, Y. Li, G. Zhao, M. Zhang, Microvesicle-mediated delivery of minicircle DNA results in effective gene-directed enzyme prodrug cancer therapy, Thorac. Cancer. 10 (2019) 1962-1972, https://doi.org/10.1111/17597714.13175 .

[73] H. Gomari, M.F. Moghadam, M. Soleimani, M. Ghavami, S. Khodashenas, Targeted delivery of doxorubicin to HER2 positive tumor models, Int. J. Nanomedicine. 14 (2019) 5679-5690, https://doi.org/10.2147/IJN.S210731.

[74] H. Cheng, J.H. Fan, L.P. Zhao, G.L. Fan, R.R. Zheng, X.Z. Qiu, X.Y. Yu, S.Y. Li, X.Z. Zhang, Chimeric peptide engineered exosomes for dual-stage light guided plasma membrane and nucleus targeted photodynamic therapy, Biomaterials. $211 \quad$ (2019) 14-24, https://doi.org/10.1016/j. biomaterials.2019.05.004.

[75] C. Melzer, V. Rehn, Y. Yang, H. Bähre, J. von der Ohe, R. Hass, Taxol-loaded MSC-Derived exosomes provide a therapeutic vehicle to target metastatic breast cancer and other carcinoma cells, Cancers. 11 (2019) 1-20, https://doi. org/10.3390/cancers11060798.

[76] P. Wang, H. Wang, Q. Huang, C. Peng, L. Yao, H. Chen, Z. Qiu, Y. Wu, L. Wang, W. Chen, Exosomes from M1-polarized macrophages enhance paclitaxel antitumor activity by activating macrophages-mediated inflammation, Theranostics. 9 (2019) 1714-1727, https://doi.org/10.7150/thno.30716.

[77] Y. Liu, L. Bai, K. Guo, Y. Jia, K. Zhang, Q. Liu, P. Wang, X. Wang, Focused ultrasound-augmented targeting delivery of nanosonosensitizers from homogenous exosomes for enhanced sonodynamic cancer therapy, Theranostics. 9 (2019) 5261-5281, https://doi.org/10.7150/thno.33183.

[78] T. Yong, X.X. Zhang, N. Bie, H. Zhang, X.X. Zhang, F. Li, A. Hakeem, J. Hu, L. Gan, H.A. Santos, X. Yang, Tumor exosome-based nanoparticles are efficient drug carriers for chemotherapy, Nat. Commun. 10 (2019) 3838, https://doi.org/ 10.1038/s41467-019-11718-4.

[79] Z. Naseri, R.K. Oskuee, M.R. Jaafari, M.F. Moghadam, Exosome-mediated delivery of functionally active miRNA-142-3p inhibitor reduces tumorigenicity of breast cancer in vitro and in vivo, Int. J. Nanomedicine. 13 (2018) 7727-7747, https://doi.org/10.2147/IJN.S182384.

[80] F. Pi, D.W. Binzel, T.J. Lee, Z. Li, M. Sun, P. Rychahou, H. Li, F. Haque, S. Wang, C. M. Croce, B. Guo, B.M. Evers, P. Guo, Nanoparticle orientation to control RNA loading and ligand display on extracellular vesicles for cancer regression, Nat. Nanotechnol. 13 (2018) 82-89, https://doi.org/10.1038/s41565-017-0012-z.

[81] Y. Wan, L. Wang, C. Zhu, Q. Zheng, G. Wang, J. Tong, Y. Fang, Y. Xia, G. Cheng, X. He, S.-Y. Zheng, Aptamer-conjugated extracellular nanovesicles for targeted drug delivery, Cancer Res. 78 (2018) 798-808, https://doi.org/ 10.1158/0008-5472.CAN-17-2880.

[82] J.H. Wang, A.V. Forterre, J. Zhao, D.O. Frimannsson, A. Delcayre, T.J. Antes, B. Efron, S.S. Jeffrey, M.D. Pegram, A.C. Matin, Anti-her2 scfv-directed extracellular vesicle-mediated mRNA-based gene delivery inhibits growth of her2-positive human breast tumor xenografts by prodrug activation, Mol. Cancer Ther. 17 (2018) 1133-1142, https://doi.org/10.1158/1535-7163.MCT17-0827.

[83] Y. Hong, G.H. Nam, E. Koh, S. Jeon, G.B. Kim, C. Jeong, D.H. Kim, Y. Yang, I.S Kim, Exosome as a vehicle for delivery of membrane protein therapeutics, $\mathrm{PH} 20$, for enhanced tumor penetration and antitumor efficacy, Adv. Funct. Mater. 28 (2018) 1-9, https://doi.org/10.1002/adfm.201703074.

[84] W.M. Usman, T.C. Pham, Y.Y. Kwok, L.T. Vu, V. Ma, B. Peng, Y.S. Chan, L. Wei, S. M. Chin, A. Azad, A.B.-L. He, A.Y.H. Leung, M. Yang, N. Shyh-Chang, W.C. Cho, J. Shi, M.T.N. Le, Efficient RNA drug delivery using red blood cell extracellular vesicles, Nat. Commun. 9 (2018) 2359, https://doi.org/10.1038/s41467-01804791-8.

[85] Y. Wang, X. Chen, B. Tian, J. Liu, L. Yang, L. Zeng, T. Chen, A. Hong, X. Wang, Nucleolin-targeted extracellular vesicles as a versatile platform for biologics delivery to breast cancer, Theranostics. 7 (2017) 1360-1372, https://doi.org/ 10.7150/thno.16532.

[86] M. Hadla, S. Palazzolo, G. Corona, I. Caligiuri, V. Canzonieri, G. Toffoli, F. Rizzolio, Exosomes increase the therapeutic index of doxorubicin in breast and ovarian cancer mouse models, Nanomedicine. 11 (2016) 2431-2441, https://doi.org/10.2217/nnm-2016-0154.

[87] G. Toffoli, M. Hadla, G. Corona, I. Caligiuri, S. Palazzolo, S. Semeraro, A. Gamini, V. Canzonieri, F. Rizzolio, Exosomal doxorubicin reduces the cardiac toxicity of doxorubicin, Nanomedicine. 10 (2015) 2963-2971, https://doi.org 10.2217/nnm.15.118.

[88] Y. Yang, Y. Chen, F. Zhang, Q. Zhao, H. Zhong, Increased anti-tumour activity by exosomes derived from doxorubicin-treated tumour cells via heat stress, Int. J. Hyperthermia. 31 (2015) 498-506, https://doi.org/10.3109/ 02656736.2015 .1036384

[89] Y. Tian, S. Li, J. Song, T. Ji, M. Zhu, G.J. Anderson, J. Wei, G. Nie, A doxorubicin delivery platform using engineered natural membrane vesicle exosomes for targeted tumor therapy, Biomaterials. 35 (2014) 2383-2390, https://doi.org/ 10.1016/j.biomaterials.2013.11.083.

[90] S. Ohno, M. Takanashi, K. Sudo, S. Ueda, A. Ishikawa, N. Matsuyama, K. Fujita, T. Mizutani, T. Ohgi, T. Ochiya, N. Gotoh, M. Kuroda, Systemically injected exosomes targeted to EGFR deliver antitumor microRNA to breast cancer cells, Mol. Ther. 21 (2013) 185-191, https://doi.org/10.1038/mt.2012.180.

[91] J. Liu, Z. Ye, M. Xiang, B. Chang, J. Cui, T. Ji, L. Zhao, Q. Li, Y. Deng, L. Xu, G. Wang, L. Wang, Z. Wang, Functional extracellular vesicles engineered with lipid-grafted hyaluronic acid effectively reverse cancer drug resistance, Biomaterials. 223 (2019) 119475, https://doi.org/10.1016/j. biomaterials.2019.119475.

[92] F. Xiong, X. Ling, X. Chen, J. Chen, J. Tan, W. Cao, L. Ge, M. Ma, J. Wu, Pursuing specific chemotherapy of orthotopic breast cancer with lung metastasis from docking nanoparticles driven by bioinspired exosomes, Nano Lett. 19 (2019) 3256-3266, https://doi.org/10.1021/acs.nanolett.9b00824.

[93] L. Zhao, C. Gu, Y. Gan, L. Shao, H. Chen, H. Zhu, Exosome-mediated siRNA delivery to suppress postoperative breast cancer metastasis, J. Control Release. 318 (2020) 1-15, https://doi.org/10.1016/j.jconrel.2019.12.005.

[94] K.O. Jung, H. Jo, J.H. Yu, S.S. Gambhir, G. Pratx, Development and MPI tracking of novel hypoxia-targeted theranostic exosomes, Biomaterials. 177 (2018) 139-148, https://doi.org/10.1016/j.biomaterials.2018.05.048.

[95] Y. Hong, Y.K. Kim, G.B. Kim, G.H. Nam, S.A. Kim, Y. Park, Y. Yang, I.S. Kim, Degradation of tumour stromal hyaluronan by small extracellular vesiclePH20 stimulates CD103+ dendritic cells and in combination with PD-L1 blockade boosts anti-tumour immunity, J. Extracell. Vesicles. 8 (2019), https://doi.org/10.1080/20013078.2019.1670893.

[96] C. Gong, J. Tian, Z. Wang, Y. Gao, X. Wu, X. Ding, L. Qiang, G. Li, Z. Han, Y. Yuan, S. Gao, Functional exosome-mediated co-delivery of doxorubicin and hydrophobically modified microRNA 159 for triple-negative breast cancer therapy, J. Nanobiotechnology. 17 (2019) 1-18, https://doi.org/10.1186/ s12951-019-0526-7.

[97] E. Bagheri, K. Abnous, S.A. Farzad, S.M. Taghdisi, M. Ramezani, M. Alibolandi, Targeted doxorubicin-loaded mesenchymal stem cells-derived exosomes as a versatile platform for fighting against colorectal cancer, Life Sci. 261 (2020) 118369, https://doi.org/10.1016/j.lfs.2020.118369.

[98] X. Wang, H. Zhang, H. Yang, M. Bai, T. Ning, T. Deng, R. Liu, Q. Fan, K. Zhu, J. Li, Y. Zhan, G. Ying, Y. Ba, Exosome-delivered circRNA promotes glycolysis to induce chemoresistance through the miR-122-PKM2 axis in colorectal cancer, Mol. Oncol. 14 (2020) 539-555, https://doi.org/10.1002/1878-0261.12629.

[99] Z. Zheng, Z. Li, C. Xu, B. Guo, P. Guo, Folate-displaying exosome mediated cytosolic delivery of siRNA avoiding endosome trapping, J. Control Release. 311-312 (2019) 43-49, https://doi.org/10.1016/j.jconrel.2019.08.021.

[100] Y. Li, Y. Gao, C. Gong, Z. Wang, Q. Xia, F. Gu, C. Hu, L. Zhang, H. Guo, S. Gao, A33 antibody-functionalized exosomes for targeted delivery of doxorubicin against colorectal cancer, Nanomedicine. 14 (2018) 1973-1985, https://doi. org/10.1016/j.nano.2018.05.020. 
[101] G. Liang, Y. Zhu, D.J. Ali, T. Tian, H. Xu, K. Si, B. Sun, B. Chen, Z. Xiao, Engineered exosomes for targeted co-delivery of miR-21 inhibitor and chemotherapeutics to reverse drug resistance in colon cancer, J. Nanobiotechnology. 18 (2020) 10, https://doi.org/10.1186/s12951-019-0563-2.

[102] E. Cho, G.-H. Nam, Y. Hong, Y.K. Kim, D.-H. Kim, Y. Yang, I.-S. Kim, Comparison of exosomes and ferritin protein nanocages for the delivery of membrane protein therapeutics, J. Control Release. 279 (2018) 326-335, https://doi.org/ 10.1016/j.jconrel.2018.04.037.

[103] E. Koh, E.J. Lee, G.H. Nam, Y. Hong, E. Cho, Y. Yang, I.S. Kim, Exosome-SIRP $\alpha$, a CD47 blockade increases cancer cell phagocytosis, Biomaterials. 121 (2017) 121-129, https://doi.org/10.1016/j.biomaterials.2017.01.004.

[104] W. Fan, X.-D. Tian, E. Huang, J.-J. Zhang, Exosomes from CIITA-transfected CT26 cells enhance anti-tumor effects, Asian Pac. J. Cancer Prev. 14 (2013) 987-991, https://doi.org/10.7314/APJCP.2013.14.2.987.

[105] M. Garofalo, A. Villa, D. Crescenti, M. Marzagalli, L. Kuryk, P. Limonta, V. Mazzaferro, P. Ciana, Heterologous and cross-species tropism of cancerderived extracellular vesicles, Theranostics. 9 (2019) 5681-5693, https://doi. org/10.7150/thno.34824.

[106] J. Peng, J. Zhao, Y. Zhao, P. Wu, L. Gou, S. Fu, P. Chen, Y. Lu, L. Yang, HeLa cellderived paclitaxel-loaded microparticles efficiently inhibit the growth of cervical carcinoma, Int. J. Nanomedicine Volume. 15 (2020) 6409-6420, https://doi.org/10.2147/IJN.S246659.

[107] J. Wang, Y. Dong, Y. Li, W. Li, K. Cheng, Y. Qian, G. Xu, X. Zhang, L. Hu, P. Chen, W. Du, X. Feng, Y.-D. Zhao, Z. Zhang, B.-F. Liu, Designer exosomes for active targeted chemo-photothermal synergistic tumor therapy, Adv. Funct. Mater. 28 (2018) 1707360, https://doi.org/10.1002/adfm.201707360.

[108] F. Aqil, R. Munagala, J. Jeyabalan, A.K. Agrawal, R. Gupta, Exosomes for the enhanced tissue bioavailability and efficacy of curcumin, AAPS. J. 19 (2017) 1691-1702, https://doi.org/10.1208/s12248-017-0154-9.

[109] W. Zhang, Z.-L. Yu, M. Wu, J.-G. Ren, H.-F. Xia, G.-L. Sa, J.-Y. Zhu, D.-W. Pang, Y.-F. Zhao, G. Chen, Magnetic and folate functionalization enables rapid isolation and enhanced tumor-targeting of cell-derived microvesicles, ACS Nano. 11 (2017) 277-290, https://doi.org/10.1021/acsnano.6b05630.

[110] A.K.A. Silva, J. Kolosnjaj-Tabi, S. Bonneau, I. Marangon, N. Boggetto, K. Aubertin, O. Clément, M.F. Bureau, N. Luciani, F. Gazeau, C. Wilhelm, Magnetic and photoresponsive theranosomes: translating cell-released vesicles into smart nanovectors for cancer therapy, ACS Nano. 7 (2013) 4954-4966, https://doi.org/10.1021/nn400269x.

[111] H. Tao, H. Xu, L. Zuo, C. Li, G. Qiao, M. Guo, L. Zheng, M. Leitgeb, X. Lin, Exosomes-coated bcl-2 siRNA inhibits the growth of digestive system tumors both in vitro and in vivo, Int. J. Biol. Macromol. 161 (2020) 470-480, https:// doi.org/10.1016/j.ijbiomac.2020.06.052.

[112] S. Pan, L. Pei, A. Zhang, Y. Zhang, C. Zhang, M. Huang, Z. Huang, B. Liu, L. Wang, L. Ma, Q. Zhang, D. Cui, Passion fruit-like exosome-PMA/Au-BSA@Ce6 nanovehicles for real-time fluorescence imaging and enhanced targeted photodynamic therapy with deep penetration and superior retention behavior in tumor, Biomaterials. 230 (2020) 119606, https://doi.org/ 10.1016/j.biomaterials.2019.119606.

[113] X. Wang, H. Zhang, M. Bai, T. Ning, S. Ge, T. Deng, R. Liu, L. Zhang, G. Ying, Y. $\mathrm{Ba}$, Exosomes serve as nanoparticles to deliver anti-miR-214 to reverse chemoresistance to cisplatin in gastric cancer, Mol. Ther. 26 (2018) 774-783, https://doi.org/10.1016/j.ymthe.2018.01.001.

[114] H. Zhang, M. Bai, T. Deng, R. Liu, X. Wang, Y. Qu, J. Duan, L. Zhang, T. Ning, S. Ge, H. Li, L. Zhou, Y. Liu, G. Ying, D. Huang, Y. Ba, Cell-derived microvesicles mediate the delivery of miR-29a/c to suppress angiogenesis in gastric carcinoma, Cancer Lett. 375 (2016) 331-339, https://doi.org/10.1016/ j.canlet.2016.03.026

[115] J. Li, N. Li, J. Wang, M1 macrophage-derived exosome-encapsulated cisplatin can enhance its anti-lung cancer effect, Minerva Med. (2020). http://doi.org/ 10.23736/S0026-4806.20.06564-7.

[116] M. Garofalo, A Villa, N. Rizzi, L. Kuryk, B. Rinner, V. Cerullo, M. Yliperttula, V. Mazzaferro, P. Ciana, Extracellular vesicles enhance the targeted delivery of immunogenic oncolytic adenovirus and paclitaxel in immunocompetent mice, J. Control Release. 294 (2019) 165-175, https://doi.org/10.1016/j. jconrel.2018.12.022.

[117] J. Ma, Y. Zhang, K. Tang, H. Zhang, X. Yin, Y. Li, P. Xu, Y. Sun, R. Ma, T. Ji, J. Chen, S. Zhang, T. Zhang, S. Luo, Y. Jin, X. Luo, C. Li, H. Gong, Z. Long, J. Lu, Z. Hu, X. Cao, N. Wang, X. Yang, B. Huang, Reversing drug resistance of soft tumorrepopulating cells by tumor cell-derived chemotherapeutic microparticles, Cell Res. 26 (2016) 713-727, https://doi.org/10.1038/cr.2016.53.

[118] F. Aqil, R. Munagala, J. Jeyabalan, A.K. Agrawal, A.H. Kyakulaga, S.A. Wilcher, R.C. Gupta, Milk exosomes - Natural nanoparticles for siRNA delivery, Cancer Lett. 449 (2019) 186-195, https://doi.org/10.1016/j.canlet.2019.02.011.

[119] M. Guo, F. Wu, G. Hu, L. Chen, J. Xu, P. Xu, X. Wang, Y. Li, S. Liu, S. Zhang, Q. Huang, J. Fan, Z. Lv, M. Zhou, L. Duan, T. Liao, G. Yang, K. Tang, B. Liu, X. Liao, X. Tao, Y. Jin, Autologous tumor cell-derived microparticle-based targeted chemotherapy in lung cancer patients with malignant pleural effusion, Sci. Transl. Med. 11 (2019) 1-16, https://doi.org/10.1126/scitranslmed.aat5690.

[120] M. Garofalo, A. Villa, N. Rizzi, L. Kuryk, V. Mazzaferro, P. Ciana, Systemic administration and targeted delivery of immunogenic oncolytic adenovirus encapsulated in extracellular vesicles for cancer therapies, Viruses. 10 (2018), https://doi.org/10.3390/v10100558.

[121] M. Garofalo, H. Saari, P. Somersalo, D. Crescenti, L. Kuryk, L. Aksela, C. Capasso, M. Madetoja, K. Koskinen, T. Oksanen, A. Mäkitie, M. Jalasvuori, V. Cerullo, P. Ciana, M. Yliperttula, Antitumor effect of oncolytic virus and paclitaxel encapsulated in extracellular vesicles for lung cancer treatment, J.
Control Release. 283 (2018) 223-234, https://doi.org/10.1016/j. jconrel.2018.05.015.

[122] A.K. Agrawal, F. Aqil, J. Jeyabalan, W.A. Spencer, J. Beck, B.W. Gachuki, S.S. Alhakeem, K. Oben, R. Munagala, S. Bondada, R.C. Gupta, Milk-derived exosomes for oral delivery of paclitaxel, Nanomedicine. 13 (2017) 16271636, https://doi.org/10.1016/j.nano.2017.03.001.

[123] R. Munagala, F. Aqil, J. Jeyabalan, A.K. Agrawal, A.M. Mudd, A.H. Kyakulaga, I. P. Singh, M.V. Vadhanam, R.C. Gupta, Exosomal formulation of anthocyanidins against multiple cancer types, Cancer Lett. 393 (2017) 94102, https://doi.org/10.1016/j.canlet.2017.02.004.

[124] R. Munagala, F. Aqil, J. Jeyabalan, R.C. Gupta, Bovine milk-derived exosomes for drug delivery, Cancer Lett. 371 (2016) 48-61, https://doi.org/10.1016/ j.canlet.2015.10.020.

[125] F. Aqil, H. Kausar, A.K. Agrawal, J. Jeyabalan, A.-H. Kyakulaga, R. Munagala, R. Gupta, Exosomal formulation enhances therapeutic response of celastrol against lung cancer, Exp. Mol. Med. 101 (2016) 12-21, https://doi.org/ 10.1016/j.yexmp.2016.05.013.

[126] M.S. Kim, M.J. Haney, Y. Zhao, V. Mahajan, I. Deygen, N.L. Klyachko, E. Inskoe, A. Piroyan, M. Sokolsky, O. Okolie, S.D. Hingtgen, A.V. Kabanov, E.V. Batrakova, Development of exosome-encapsulated paclitaxel to overcome MDR in cancer cells, Nanomedicine. 12 (2016) 655-664, https://doi.org/ 10.1016/j.nano.2015.10.012.

[127] H. Nie, X. Xie, D. Zhang, Y. Zhou, B. Li, F.F. Li, F.F. Li, Y. Cheng, H. Mei, H. Meng, L. Jia, Use of lung-specific exosomes for miRNA-126 delivery in non-small cell lung cancer, Nanoscale. 12 (2020) 877-887, https://doi.org/10.1039/ c9nr09011h.

[128] Q. Lin, M. Qu, B. Zhou, H.K. Patra, Z. Sun, Q. Luo, W. Yang, Y. Wu, Y. Zhang, L. Li, L. Deng, L. Wang, T. Gong, Q. He, L. Zhang, X. Sun, Z. Zhang, Exosome-like nanoplatform modified with targeting ligand improves anti-cancer and antiinflammation effects of imperialine, J. Control Release. 311-312 (2019) 104116, https://doi.org/10.1016/j.jconrel.2019.08.037.

[129] M.S. Kim, M.J. Haney, Y. Zhao, D. Yuan, I. Deygen, N.L. Klyachko, A.V. Kabanov, E.V. Batrakova, Engineering macrophage-derived exosomes for targeted paclitaxel delivery to pulmonary metastases: in vitro and in vivo evaluations, Nanomedicine. 14 (2018) 195-204, https://doi.org/10.1016/ j.nano.2017.09.011.

[130] D. Hao, Y. Li, G. Zhao, M. Zhang, Soluble fms-like tyrosine kinase-1-enriched exosomes suppress the growth of small cell lung cancer by inhibiting endothelial cell migration, Thorac. Cancer. 10 (2019) 1962-1972, https://doi. org/10.1111/1759-7714.13175.

[131] G. Lou, L. Chen, C. Xia, W. Wang, J. Qi, A. Li, L. Zhao, Z. Chen, M. Zheng, Y. Liu, MiR-199a-modified exosomes from adipose tissue-derived mesenchymal stem cells improve hepatocellular carcinoma chemosensitivity through mTOR pathway, J. Exp. Clin. Cancer. Res. 39 (2020) 1-9, https://doi.org/ 10.1186/s13046-019-1512-5.

[132] D. Wang, Y. Yao, J. He, X. Zhong, B. Li, S. Rao, H. Yu, S. He, X. Feng, T. Xu, B. Yang, T. Yong, L. Gan, J. Hu, X. Yang, Engineered cell-derived microparticles Bi 2 Se 3 /DOX@MPs for imaging guided synergistic photothermal/low-dose chemotherapy of cancer, Adv. Sci. 7 (2020) 1901293, https://doi.org/10.1002/ advs.201901293.

[133] H. Qi, C. Liu, L. Long, Y. Ren, S. Zhang, X. Chang, X. Qian, H. Jia, J. Zhao, J. Sun, X. Hou, X. Yuan, C. Kang, Blood exosomes endowed with magnetic and targeting properties for cancer therapy, ACS Nano. 10 (2016) 3323-3333, https://doi. org/10.1021/acsnano.5b06939.

[134] K. Tang, Y. Zhang, H. Zhang, P. Xu, J. Liu, J. Ma, M. Lv, D. Li, F. Katirai, G.-X Shen, G. Zhang, Z.-H. Feng, D. Ye, B. Huang, Delivery of chemotherapeutic drugs in tumour cell-derived microparticles, Nat. Commun. 3 (2012) 1282, https://doi.org/10.1038/ncomms2282.

[135] T. Wu, Y. Qi, D. Zhang, Q. Song, C. Yang, X. Hu, Y. Bao, Y. Zhao, Z. Zhang, Bone marrow dendritic cells derived microvesicles for combinational immunochemotherapy against tumor, Adv. Funct. Mater. 27 (2017) 1703191, https://doi.org/10.1002/adfm.201703191.

[136] L. Rivoltini, C. Chiodoni, P. Squarcina, M. Tortoreto, A. Villa, B. Vergani, M. Bürdek, L. Botti, I. Arioli, A. Cova, G. Mauri, E. Vergani, B. Bianchi, P. Della Mina, L. Cantone, V. Bollati, N. Zaffaroni, A.M. Gianni, M.P. Colombo, V. Huber, TNF-related apoptosis-inducing ligand (TRAIL)-armed exosomes deliver proapoptotic signals to tumor site, Clin. Cancer Res. 22 (2016) 3499-3512, https://doi.org/10.1158/1078-0432.CCR-15-2170.

[137] D. Ingato, J.A. Edson, M. Zakharian, Y.J. Kwon, Cancer cell-derived, drugloaded nanovesicles induced by sulfhydryl-blocking for effective and safe cancer therapy, ACS Nano. 12 (2018) 9568-9577, https://doi.org/10.1021/ acsnano.8b05377.

[138] M. Zhuang, X. Chen, D. Du, J. Shi, M. Deng, Q. Long, X. Yin, Y. Wang, L. Rao, SPION decorated exosome delivery of TNF- $\alpha$ to cancer cell membranes through magnetism, Nanoscale. 12 (2020) 173-188, https://doi.org/10.1039/ c9nr05865f.

[139] F.H. Shamili, H.R. Bayegi, Z. Salmasi, K. Sadri, M. Mahmoudi, M. Kalantari, M. Ramezani, K. Abnous, Exosomes derived from TRAIL-engineered mesenchymal stem cells with effective anti-tumor activity in a mouse melanoma model, Int. J. Pharm. 549 (2018) 218-229, https://doi.org/ 10.1016/j.ijpharm.2018.07.067.

[140] M. Morishita, Y. Takahashi, A. Matsumoto, M. Nishikawa, Y. Takakura, Exosome-based tumor antigens-adjuvant co-delivery utilizing genetically engineered tumor cell-derived exosomes with immunostimulatory CpG DNA,

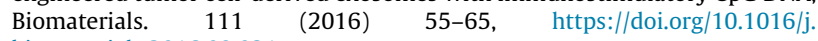
biomaterials.2016.09.031. 
[141] G. Chen, J.-Y. Zhu, Z.-L. Zhang, W. Zhang, J.-G. Ren, M. Wu, Z.-Y. Hong, C. Lv, D.-W. Pang, Y.-F. Zhao, Transformation of cell-derived microparticles into quantum-dot-labeled nanovectors for antitumor siRNA delivery, Angew. Chem. Int. Ed. Engl. 54 (2015) 1036-1040, https://doi.org/10.1002/ anie.201410223.

[142] H. Liu, M. Shen, D. Zhao, D. Ru, Y. Duan, C. Ding, H. Li, The effect of triptolideloaded exosomes on the proliferation and apoptosis of human ovarian cancer SKOV3 cells, Biomed. Res. Int. 2019 (2019) 1-14, https://doi.org/10.1155/ 2019/2595801.

[143] S.M. Kim, Y. Yang, S.J. Oh, Y. Hong, M. Seo, M. Jang, Cancer-derived exosomes as a delivery platform of CRISPR/Cas9 confer cancer cell tropism-dependent targeting, J. Control Release. 266 (2017) 8-16, https://doi.org/10.1016/j. jconrel.2017.09.013.

[144] F. Aqil, J. Jeyabalan, A.K. Agrawal, A.-H. Kyakulaga, R. Munagala, L. Parker, R.C. Gupta, Exosomal delivery of berry anthocyanidins for the management of ovarian cancer, Food Funct. 8 (2017) 4100-4107, https://doi.org/10.1039/ C7FO00882A.

[145] L. Xu, F.N. Faruqu, Y.M. Lim, K.Y. Lim, R. Liam-Or, A.A. Walters, P. Lavender, D. Fear, C.M. Wells, J. Tzu-Wen Wang, K.T. Al-Jamal, Exosome-mediated RNAi of PAK4 prolongs survival of pancreatic cancer mouse model after loco-regional treatment, Biomaterials. 264 (2021) 120369, https://doi.org/10.1016/j. biomaterials. 2020.120369.

[146] Y.J. Li, J.Y. Wu, J.M. Wang, X.B. Hu, J.X. Cai, D.X. Xiang, Gemcitabine loaded autologous exosomes for effective and safe chemotherapy of pancreatic cancer, Acta. Biomater. 101 (2020) 519-530, https://doi.org/10.1016/j. actbio. 2019.10.022.

[147] M. Mendt, S. Kamerkar, H. Sugimoto, K.M. McAndrews, C.-C. Wu, M. Gagea, S. Yang, E.V.R. Blanko, Q. Peng, X. Ma, J.R. Marszalek, A. Maitra, C. Yee, K. Rezvani, E. Shpall, V.S. LeBleu, R. Kalluri, Generation and testing of clinicalgrade exosomes for pancreatic cancer, JCI Insight. 3 (2018) 1-22, https://doi. org/10.1172/jci.insight.99263.

[148] S. Kamerkar, V.S. LeBleu, H. Sugimoto, S. Yang, C.F. Ruivo, S.A. Melo, J.J. Lee, R. Kalluri, Exosomes facilitate therapeutic targeting of oncogenic KRAS in pancreatic cancer, Nature. 546 (2017) 498-503, https://doi.org/ 10.1038 /nature22341.

[149] Y. Ding, F. Cao, H. Sun, Y. Wang, S. Liu, Y. Wu, Q. Cui, W.T. Mei, F. Li, Exosomes derived from human umbilical cord mesenchymal stromal cells deliver exogenous miR-145-5p to inhibit pancreatic ductal adenocarcinoma progression, Cancer Lett. 442 (2019) 351-361, https://doi.org/10.1016/ j.canlet.2018.10.039.

[150] Y. Binenbaum, E. Fridman, Z. Yaari, N. Milman, A. Schroeder, G. Ben David, T. Shlomi, Z. Gil, Transfer of miRNA in macrophage-derived exosomes induces drug resistance in pancreatic adenocarcinoma, Cancer Res. 78 (2018) 52875299, https://doi.org/10.1158/0008-5472.CAN-18-0124.

[151] L. Qiao, S. Hu, K. Huang, T. Su, Z. Li, A. Vandergriff, J. Cores, P.U. Dinh, T. Allen, D. Shen, H. Liang, Y. Li, K. Cheng, Tumor cell-derived exosomes home to their cells of origin and can be used as Trojan horses to deliver cancer drugs, Theranostics. 10 (2020) 3474-3487, https://doi.org/10.7150/thno.39434.

[152] Y.Y. Zhang, L. Li, J. Yu, D. Zhu, Y.Y. Zhang, X. Li, H. Gu, C.Y. Zhang, K. Zen, Microvesicle-mediated delivery of transforming growth factor $\beta 1$ siRNA for the suppression of tumor growth in mice, Biomaterials. 35 (2014) 43904400, https://doi.org/10.1016/j.biomaterials.2014.02.003.

[153] J. Li, Y. Zhang, Y. Liu, X. Dai, W. Li, X. Cai, Y. Yin, Q. Wang, Y. Xue, C. Wang, D. Li, D. Hou, X. Jiang, J. Zhang, K. Zen, X. Chen, C.-Y. Zhang, Microvesiclemediated transfer of MicroRNA-150 from monocytes to endothelial cells promotes angiogenesis, J. Biol. Chem. 288 (2013) 23586-23596, https://doi. org/10.1074/jbc.M113.489302.

[154] Z. Li, H. Wang, H. Yin, C. Bennett, H.G. Zhang, P. Guo, Arrowtail RNA for ligand display on ginger exosome-like nanovesicles to systemic deliver siRNA for cancer suppression, Sci. Rep. 8 (2018) 1-11, https://doi.org/10.1038/s41598018-32953-7.

[155] D. Bellavia, S. Raimondo, G. Calabrese, S. Forte, M. Cristaldi, A. Patinella, L. Memeo, M. Manno, S. Raccosta, P. Diana, G. Cirrincione, G. Giavaresi, F. Monteleone, S. Fontana, G.D. Leo, R. Alessandro, Interleukin 3- receptor targeted exosomes inhibit in vitro and in vivo chronic myelogenous Leukemia cell growth, Theranostics. 7 (2017) 1333-1345, https://doi.org/ $10.7150 /$ thno.17092.

[156] J. Wang, Q. Jiang, O.D. Faleti, C.M. Tsang, M. Zhao, G. Wu, S.W. Tsao, M. Fu, Y. Chen, T. Ding, T. Chong, Y. Long, X. Yang, Y. Zhang, Y. Cai, H. Li, M. Peng, X. Lyu, $\mathrm{X}$. Li, Exosomal delivery of antagomirs targeting viral and cellular microRNAs synergistically inhibits cancer angiogenesis, Mol. Ther. Nucleic Acids. 22 (2020) 153-165, https://doi.org/10.1016/j.omtn.2020.08.017.

[157] Y. Si, S. Kim, E. Zhang, Y. Tang, R. Jaskula-Sztul, J.M. Markert, H. Chen, L. Zhou, $\mathrm{X}$. Liu, Targeted exosomes for drug delivery: biomanufacturing, surface tagging, and validation, Biotechnol. J. 15 (2020) 1900163, https://doi.org/ 10.1002/biot.201900163.

[158] J.J. He, J.J. He, L. Min, Y. He, H. Guan, J. Wang, X. Peng, Extracellular vesicles transmitted miR-31-5p promotes sorafenib resistance by targeting MLH1 in renal cell carcinoma, Int. J. Cancer. 146 (2020) 1052-1063, https://doi.org/ $10.1002 /$ ijc.32543.

[159] A. Mizrak, M.F. Bolukbasi, G.B. Ozdener, G.J. Brenner, S. Madlener, E.P. Erkan, T. Ströbel, X.O. Breakefield, O. Saydam, Genetically engineered microvesicles carrying suicide mRNA/protein inhibit schwannoma tumor growth, Mol. Ther. 21 (2013) 101-108, https://doi.org/10.1038/mt.2012.161.

[160] P. Huang, L. Wang, Q. Li, X. Tian, J. Xu, J. Xu, Y. Xiong, G. Chen, H. Qian, C. Jin, Y. Yu, K. Cheng, L. Qian, Y. Yang, Atorvastatin enhances the therapeutic efficacy of mesenchymal stem cells-derived exosomes in acute myocardial infarction via up-regulating long non-coding RNA H19, Cardiovasc. Res. 116 (2019) 353-367, https://doi.org/10.1093/cvr/cvz139.

[161] Q. Luo, D. Guo, G. Liu, G. Chen, M. Hang, M. Jin, Exosomes from MiR-126overexpressing adscs are therapeutic in relieving acute myocardial ischaemic injury, Cell Physiol. Biochem. 44 (2018) 2105-2116, https://doi.org/10.1159/ 000485949.

[162] J. Ma, Y. Zhao, L. Sun, X.X. Sun, X. Zhao, X.X. Sun, H. Qian, W. Xu, W. Zhu, Exosomes derived from Akt -modified human umbilical cord mesenchymal stem cells improve cardiac regeneration and promote angiogenesis via activating platelet-derived growth factor D, Stem Cells Transl. Med. 6 (2017) 51-59, https://doi.org/10.5966/sctm.2016-0038.

[163] J. Ni, X. Liu, Y. Yin, P. Zhang, Y.W. Xu, Z. Liu, Exosomes derived from TIMP2modified human umbilical cord mesenchymal stem cells enhance the repair effect in rat model with myocardial infarction possibly by the Akt/ SFRP2 pathway, Oxid. Med. Cell. Longev. 2019 (2019), https://doi.org/10.1155/ 2019/1958941.

[164] Y. Song, C. Zhang, J. Zhang, Z. Jiao, N. Dong, G. Wang, Z. Wang, L. Wang, Localized injection of miRNA-21-enriched extracellular vesicles effectively restores cardiac function after myocardial infarction, Theranostics. 9 (2019) 2346-2360, https://doi.org/10.7150/thno.29945.

[165] J.Y. Kang, H.H. Park, H. Kim, D. Mun, H.H. Park, N. Yun, B. Joung, Human peripheral blood-derived exosomes for microRNA delivery, Int. J. Mol. Med. 43 (2019) 2319-2328, https://doi.org/10.3892/ijmm.2019.4150.

[166] X.H. Gong, H. Liu, S.J. Wang, S.W. Liang, G.G. Wang, Exosomes derived from SDF1-overexpressing mesenchymal stem cells inhibit ischemic myocardial cell apoptosis and promote cardiac endothelial microvascular regeneration in mice with myocardial infarction, J. Cell Physiol. 234 (2019) 13878-13893, https://doi.org/10.1002/jcp.28070.

[167] Q. Chen, Y. Liu, X. Ding, Q. Li, F. Qiu, M. Wang, Z. Shen, H. Zheng, G. Fu, Bone marrow mesenchymal stem cell-secreted exosomes carrying microRNA-125b protect against myocardial ischemia reperfusion injury via targeting SIRT7, Mol. Cell. Biochem. 465 (2020) 103-114, https://doi.org/10.1007/s11010019-03671-Z.

[168] Y. Zhao, Y. Gan, G. Xu, K. Hua, D. Liu, Exosomes from MSCs overexpressing microRNA-223-3p attenuate cerebral ischemia through inhibiting microglial M1 polarization mediated inflammation, Life Sci. 260 (2020) 118403, https:// doi.org/10.1016/j.lfs.2020.118403.

[169] T. Tian, H.-X. Zhang, C.-P. He, S. Fan, Y.-L. Zhu, C. Qi, N.-P. Huang, Z.-D. Xiao, Z.H. Lu, B.A. Tannous, J. Gao, Surface functionalized exosomes as targeted drug delivery vehicles for cerebral ischemia therapy, Biomaterials. 150 (2018) 137-149, https://doi.org/10.1016/j.biomaterials.2017.10.012.

[170] J. Yang, S. Wu, L. Hou, D. Zhu, S. Yin, G. Yang, Y. Wang, Therapeutic effects of simultaneous delivery of nerve growth factor mRNA and protein via exosomes on cerebral ischemia, Mol. Ther. Nucleic Acids. 21 (2020) 512522, https://doi.org/10.1016/j.omtn.2020.06.013.

[171] J. Yang, X. Zhang, X. Chen, L. Wang, G. Yang, Exosome mediated delivery of miR-124 promotes neurogenesis after ischemia, Mol. Ther. Nucleic Acids. 7 (2017) 278-287, https://doi.org/10.1016/j.omtn.2017.04.010.

[172] R. He, Y. Jiang, Y. Shi, J. Liang, L. Zhao, Curcumin-laden exosomes target ischemic brain tissue and alleviate cerebral ischemia-reperfusion injury by inhibiting ROS-mediated mitochondrial apoptosis, Mater. Sci. Eng. C. 117 (2020), https://doi.org/10.1016/j.msec.2020.111314.

[173] X. Huang, J. Ding, Y. Li, W. Liu, J. Ji, H. Wang, X. Wang, Exosomes derived from PEDF modified adipose-derived mesenchymal stem cells ameliorate cerebral ischemia-reperfusion injury by regulation of autophagy and apoptosis, Exp. Cell Res. 371 (2018) 269-277, https://doi.org/10.1016/j.yexcr.2018.08.021.

[174] A. Kalani, P. Chaturvedi, P.K. Kamat, C. Maldonado, P. Bauer, I.G. Joshua, S.C. Tyagi, N. Tyagi, Curcumin-loaded embryonic stem cell exosomes restored neurovascular unit following ischemia-reperfusion injury, Int. J. Biochem. Cell Biol. 79 (2016) 360-369, https://doi.org/10.1016/j.biocel.2016.09.002.

[175] C. Han, J. Zhou, B. Liu, C. Liang, X. Pan, Y. Zhang, Y. Zhang, Y. Wang, L. Shao, B. Zhu, J. Wang, Q. Yin, X.Y. Yu, Y. Li, Delivery of miR-675 by stem cell-derived exosomes encapsulated in silk fibroin hydrogel prevents aging-induced vascular dysfunction in mouse hindlimb, Mater. Sci. Eng. C. 99 (2019) 322332, https://doi.org/10.1016/j.msec.2019.01.122.

[176] W. Sun, P. Zhao, Y. Zhou, C. Xing, L. Zhao, Z. Li, L. Yuan, Ultrasound targeted microbubble destruction assisted exosomal delivery of miR-21 protects the heart from chemotherapy associated cardiotoxicity, Biochem. Biophys. Res. Commun. 532 (2020) 60-67, https://doi.org/10.1016/j.bbrc.2020.05.044.

[177] X. Wang, H. Gu, W. Huang, J. Peng, Y. Li, L. Yang, D. Qin, K. Essandoh, Y. Wang, T. Peng, G.-C. Fan, Hsp20-mediated activation of exosome biogenesis in cardiomyocytes improves cardiac function and angiogenesis in diabetic mice, Diabetes. 65 (2016) 3111-3128, https://doi.org/10.2337/db15-1563.

[178] R. Reshke, J.A. Taylor, A. Savard, H. Guo, L.H. Rhym, P.S. Kowalski, M.T. Trung, C. Campbell, W. Little, D.G. Anderson, D. Gibbings, Reduction of the therapeutic dose of silencing RNA by packaging it in extracellular vesicles via a pre-microRNA backbone, Nat. Biomed. Eng. 4 (2020) 52-68, https://doi. org/10.1038/s41551-019-0502-4.

[179] H. Wang, H. Sui, Y. Zheng, Y. Jiang, Y. Shi, J. Liang, L. Zhao, Curcumin-primed exosomes potently ameliorate cognitive function in AD mice by inhibiting hyperphosphorylation of the Tau protein through the AKT/GSK-3 $\beta$ pathway, Nanoscale. 11 (2019) 7481-7496, https://doi.org/10.1039/C9NR01255A.

[180] L. Alvarez-Erviti, Y. Seow, H. Yin, C. Betts, S. Lakhal, M.J. Wood, Delivery of siRNA to the mouse brain by systemic injection of targeted exosomes, Nat. Biotechnol. 29 (2011) 341-345, https://doi.org/10.1038/nbt.1807. 
[181] M. Izco, J. Blesa, M. Schleef, M. Schmeer, R. Porcari, R. Al-shawi, S. Ellmerich, M.D. Toro, C. Gardiner, Y. Seow, A. Reinares-sebastian, R. Forcen, J.P. Simons, V. Bellotti, J.M. Cooper, L. Alvarez-erviti, Systemic exosomal delivery of shRNA minicircles prevents parkinsonian pathology, Mol. Ther. 27 (2019) 112, https://doi.org/10.1016/j.ymthe.2019.08.010.

[182] X. Ren, Y. Zhao, F. Xue, Y. Zheng, H. Huang, W. Wang, Y. Chang, H. Yang, J. Zhang, Exosomal DNA aptamer targeting $\alpha$-synuclein aggregates reduced neuropathological deficits in a mouse Parkinson's disease model, Mol. Ther. Nucleic Acids. 17 (2019) 726-740, https://doi.org/10.1016/j. omtn.2019.07.008.

[183] R. Kojima, D. Bojar, G. Rizzi, G.C.E. Hamri, M.D. El-Baba, P. Saxena, S. Ausländer, K.R. Tan, M. Fussenegger, Designer exosomes produced by implanted cells intracerebrally deliver therapeutic cargo for Parkinson's disease treatment, Nat. Commun. 9 (2018), https://doi.org/10.1038/s41467018-03733-8.

[184] M. Qu, Q. Lin, L. Huang, Y.Y. Fu, L. Wang, S. He, Y.Y. Fu, S. Yang, Z. Zhang, L. Zhang, X. Sun, Dopamine-loaded blood exosomes targeted to brain for better treatment of Parkinson's disease, J. Control Release. 287 (2018) 156-166, https://doi.org/10.1016/j.jconrel.2018.08.035.

[185] M.J. Haney, N.L. Klyachko, Y. Zhao, R. Gupta, E.G. Plotnikova, Z. He, T. Patel, A. Piroyan, M. Sokolsky, A.V. Kabanov, E.V. Batrakova, Exosomes as drug delivery vehicles for Parkinson's disease therapy, J Control Release. 207 (2015) 18-30, https://doi.org/10.1016/j.jconrel.2015.03.033.

[186] J.M. Cooper, P.B.O. Wiklander, J.Z. Nordin, R. Al-Shawi, M.J. Wood, M. Vithlani, A.H.V. Schapira, J.P. Simons, S. El-Andaloussi, L. Alvarez-Erviti, Systemic exosomal siRNA delivery reduced alpha-synuclein aggregates in brains of transgenic mice, Mov. Disord. 29 (2014) 1476-1485, https://doi.org/10.1002/ mds. 25978 .

[187] M.C. Didiot, L.M. Hall, A.H. Coles, R.A. Haraszti, B.M. Godinho, K. Chase, E. Sapp, S. Ly, J.F. Alterman, M.R. Hassler, D. Echeverria, L. Raj, D.V. Morrissey, M. DiFiglia, N. Aronin, A. Khvorova, Exosome-mediated delivery of hydrophobically modified siRNA for huntingtin mRNA silencing, Mol. Ther. 24 (2016) 1836-1847, https://doi.org/10.1038/mt.2016.126.

[188] F. Hosseini Shamili, M. Alibolandi, H. Rafatpanah, K. Abnous, M. Mahmoudi, M. Kalantari, S.M. Taghdisi, M. Ramezani, Immunomodulatory properties of MSC-derived exosomes armed with high affinity aptamer toward mylein as a platform for reducing multiple sclerosis clinical score, J. Control Release. 299 (2019) 149-164, https://doi.org/10.1016/j.jconrel.2019.02.032.

[189] S.C. Tao, T. Yuan, Y.L. Zhang, W.J. Yin, S.C. Guo, C.Q. Zhang, Exosomes derived from miR-140-5p-overexpressing human synovial mesenchymal stem cells enhance cartilage tissue regeneration and prevent osteoarthritis of the knee in a rat model, Theranostics. 7 (2017) 180-195, https://doi.org/10.7150/ thno.17133.

[190] Y. Liang, X. Xu, X. Li, J. Xiong, B. Li, L. Duan, D. Wang, J. Xia, Chondrocytetargeted microRNA delivery by engineered exosomes toward a cell-free osteoarthritis therapy, ACS. Appl. Mater. Interfaces. 12 (2020) 36938-36947, https://doi.org/10.1021/acsami.0c10458.

[191] Z. Chen, H. Wang, Y. Xia, F. Yan, Y. Lu, Therapeutic Potential of mesenchymal cell-derived miRNA-150-5p-expressing exosomes in rheumatoid arthritis mediated by the modulation of MMP14 and VEGF, J. Immunol. 201 (2018) 2472-2482, https://doi.org/10.4049/jimmunol.1800304.

[192] K. Bryniarski, W. Ptak, A. Jayakumar, K. Pullmann, M.J. Caplan, A. Chairoungdua, J. Lu, B.D. Adams, E. Sikora, K. Nazimek, S. Marquez, S.H. Kleinstein, P. Sangwung, Y. Iwakiri, E. Delgato, F. Redegeld, B.R. Blokhuis, J. Wojcikowski, A.W. Daniel, T. Groot Kormelink, P.W. Askenase, Antigenspecific, antibody-coated, exosome-like nanovesicles deliver suppressor Tcell microRNA-150 to effector T cells to inhibit contact sensitivity, J. Allergy Clin. Immunol. 132 (2013) 170-181, https://doi.org/10.1016/ j.jaci.2013.04.048.

[193] X. Zhuang, X. Xiang, W. Grizzle, D. Sun, S. Zhang, R.C. Axtell, S. Ju, J. Mu, L. Zhang, L. Steinman, D. Miller, H.-G. Zhang, Treatment of brain inflammatory diseases by delivering exosome encapsulated anti-inflammatory drugs from the nasal region to the brain, Mol. Ther. 19 (2011) 1769-1779, https://doi. org/10.1038/mt.2011.164.

[194] J.P. de Rivero Vaccari, F. Brand, S. Adamczak, S.W. Lee, J. Perez-Barcena, M.Y. Wang, M.R. Bullock, W.D. Dietrich, R.W. Keane, Exosome-mediated inflammasome signaling after central nervous system injury, J. Neurochem. 136 (2016) 39-48, https://doi.org/10.1111/jnc.13036.

[195] J. Yang, C.-Z. Zhou, R. Zhu, H. Fan, X.-X. Liu, X.-Y. Duan, Q. Tang, Z.-X. Shou, D.M. Zuo, miR-200b-containing microvesicles attenuate experimental colitis associated intestinal fibrosis by inhibiting epithelial-mesenchymal transition, J. Gastroenterol. Hepatol. 32 (2017) 1966-1974, https://doi.org/ 10.1111/jgh.13797.

[196] D. Sun, X. Zhuang, X. Xiang, Y. Liu, S. Zhang, C. Liu, S. Barnes, W. Grizzle, D. Miller, H.-G. Zhang, A novel nanoparticle drug delivery system: the antiinflammatory activity of curcumin is enhanced when encapsulated in exosomes, Mol. Ther. 18 (2010) 1606-1614, https://doi.org/10.1038/ mt.2010.105.

[197] X. Yang, S. Meng, H. Jiang, T. Chen, W. Wu, Exosomes derived from interleukin-10-treated dendritic cells can inhibit trinitrobenzene sulfonic acid-induced rat colitis, Scand. J. Gastroenterol. 45 (2010) 1168-1177, https://doi.org/10.3109/00365521.2010.490596.

[198] D. Wen, Y. Peng, D. Liu, Y. Weizmann, R.I. Mahato, Mesenchymal stem cell and derived exosome as small RNA carrier and Immunomodulator to improve islet transplantation, J. Control Release. 238 (2016) 166-175, https://doi.org/ 10.1016/j.jconrel.2016.07.044.
[199] W. Sun, C. Xing, L. Zhao, P. Zhao, G. Yang, L. Yuan, Ultrasound assisted exosomal delivery of tissue responsive mRNA for enhanced efficacy and minimized off-target effects, Mol. Ther. Nucleic Acids. 20 (2020) 558-567, https://doi.org/10.1016/j.omtn.2020.03.016.

[200] R.H. Nanjundappa, R. Wang, Y. Xie, C.S. Umeshappa, J. Xiang, Novel CD8 + T cell-based vaccine stimulates Gp120-specific CTL responses leading to therapeutic and long-term immunity in transgenic HLA-A2 mice, Vaccine. 30 (2012) 3519-3525, https://doi.org/10.1016/j.vaccine.2012.03.075.

[201] Y. Cheng, J.S. Schorey, Extracellular vesicles deliver Mycobacterium RNA to promote host immunity and bacterial killing, EMBO Rep. 20 (2019) 1-16. http://doi.org/10.15252/embr.201846613.

[202] M. Tapparo, S. Bruno, F. Collino, G. Togliatto, M.C. Deregibus, P. Provero, S. Wen, P.J. Quesenberry, G. Camussi, Renal regenerative potential of extracellular vesicles derived from miRNA-engineered mesenchymal stromal cells, Int. J. Mol. Sci. 20 (2019) 2381, https://doi.org/10.3390/ijms20102381.

[203] H. Wang, B. Wang, A. Zhang, F. Hassounah, Y. Seow, M. Wood, F. Ma, J.D. Klein, S.R. Price, X.H. Wang, Exosome-mediated miR-29 transfer reduces muscle atrophy and kidney fibrosis in mice, Mol. Ther. 27 (2019) 571-583, https:// doi.org/10.1016/j.ymthe.2019.01.008.

[204] Y. Wang, X. Lu, J. He, W. Zhao, Influence of erythropoietin on microvesicles derived from mesenchymal stem cells protecting renal function of chronic kidney disease, Stem Cell Res. Ther. 6 (2015) 1-14, https://doi.org/10.1186/ s13287-015-0095-0.

[205] S. Zhang, L. Jiang, H. Hu, H. Wang, X. Wang, J. Jiang, Y. Ma, J. Yang, Y. Hou, D. Xie, Q. Zhang, Pretreatment of exosomes derived from hUCMSCs with TNF- $\alpha$ ameliorates acute liver failure by inhibiting the activation of NLRP3 in macrophage, Life Sci. 246 (2020) 117401, https://doi.org/10.1016/j. lfs.2020.117401.

[206] D. Lainšček, L. Kadunc, M.M. Keber, I.H. Bratkovič, R. Romih, R. Jerala, Delivery of an artificial transcription regulator dCas9-VPR by extracellular vesicles for therapeutic gene activation, ACS. Synth. Biol. 7 (2018) 2715-2725, https:// doi.org/10.1021/acssynbio.8b00192.

[207] Z. Li, X. Zhou, M. Wei, X. Gao, L. Zhao, R. Shi, W. Sun, Y. Duan, G. Yang, L. Yuan, In vitro and in vivo RNA inhibition by CD9-HuR functionalized exosomes encapsulated with miRNA or CRISPR/dCas9, Nano Lett. 19 (2019) 19-28, https://doi.org/10.1021/acs.nanolett.8b02689.

[208] Q. Pan, V. Ramakrishnaiah, S. Henry, S. Fouraschen, P.E. de Ruiter, J. Kwekkeboom, H.W. Tilanus, H.L.A. Janssen, L.J.W. van der Laan, Hepatic cell-to-cell transmission of small silencing RNA can extend the therapeutic reach of RNA interference (RNAi), Gut. 61 (2012) 1330-1339, https://doi.org/ 10.1136/gutjnl-2011-300449.

[209] N. Ran, X. Gao, X. Dong, J. Li, C. Lin, M. Geng, H. Yin, Effects of exosomemediated delivery of myostatin propeptide on functional recovery of $\mathrm{mdx}$ mice, Biomaterials. 236 (2020) 119826, https://doi.org/10.1016/j. biomaterials.2020.119826.

[210] X. Gao, N. Ran, X. Dong, B. Zuo, R. Yang, Q. Zhou, H.M. Moulton, Y. Seow, H. Yin, Anchor peptide captures, targets, and loads exosomes of diverse origins for diagnostics and therapy, Sci. Transl. Med. 10 (2018) eaat0195, https://doi. org/10.1126/scitranslmed.aat0195.

[211] S.-C. Tao, S.-C. Guo, M. Li, Q.-F. Ke, Y.-P. Guo, C.-Q. Zhang, Chitosan wound dressings incorporating exosomes derived from MicroRNA-126overexpressing synovium mesenchymal stem cells provide sustained release of exosomes and heal full-thickness skin defects in a diabetic rat model, Stem Cells Transl. Med. 6 (2017) 736-747, https://doi.org/10.5966/ sctm.2016-0275.

[212] S.S. Yerneni, S. Lathwal, P. Shrestha, H. Shirwan, K. Matyjaszewski, L. Weiss, E. S. Yolcu, P.G. Campbell, S.R. Das, Rapid on-demand extracellular vesicle augmentation with versatile oligonucleotide tethers, ACS Nano. 13 (2019) 10555-10565, https://doi.org/10.1021/acsnano.9b04651.

[213] M. Bader, Rat Models of Cardiovascular Diseases, 2010, pp. 403-414, http://doi.org/10.1007/978-1-60327-389-3_27.

[214] M.E. Castañeda-Lopez, I. Garza-Veloz, J.M. Ortiz-Rodriguez, R. CastañedaMiranda, L.O. Solis-Sanchez, H.R. Vega-Carrillo, M.d.R. Martinez-Blanco, F. Trejo-Vazquez, G. Ornelas-Vargas, I.P. Rodriguez-Sanchez, H.A. GuerreroOsuna, I. Delgado-Enciso, O.G. Meza-Zavala, M.d.l.L. Martinez-Fierro, Animal Models of Rheumatoid Arthritis, InTech, 2018, http://doi.org/10.5772/ intechopen.72554.

[215] Bartůněk Hason, Zebrafish models of cancer-new insights on modeling human cancer in a non-mammalian vertebrate, Genes. 10 (2019) 935 https://doi.org/10.3390/genes10110935.

[216] S.E. Emam, A.S. Abu Lila, N.E. Elsadek, H. Ando, T. Shimizu, K. Okuhira, Y. Ishima, M.A. Mahdy, F.-e.S. Ghazy, T. Ishida, Cancer cell-type tropism is one of crucial determinants for the efficient systemic delivery of cancer cell-derived exosomes to tumor tissues, Eur. J. Pharm. Biopharm. 145 (2019) 27-34, https://doi.org/10.1016/j.ejpb.2019.10.005.

[217] J.M. Gudbergsson, K. Jønsson, J.B. Simonsen, K.B. Johnsen, Systematic review of targeted extracellular vesicles for drug delivery - Considerations on methodological and biological heterogeneity, J. Control Release. 306 (2019) 108-120, https://doi.org/10.1016/j.jconrel.2019.06.006.

[218] R. Huey, S. Hawthorne, P. McCarron, The potential use of rabies virus glycoprotein-derived peptides to facilitate drug delivery into the central nervous system: a mini review, J. Drug Target. 25 (2017) 379-385, https:// doi.org/10.1080/1061186X.2016.1223676.

[219] E. van der Pol, A.N. Boing, P. Harrison, A. Sturk, R. Nieuwland, Classification, functions, and clinical relevance of extracellular vesicles, Pharmacol. Rev. 64 (2012) 676-705, https://doi.org/10.1124/pr.112.005983. 
[220] E. Van Der Pol, A.G. Hoekstra, A. Sturk, C. Otto, T.G. Van Leeuwen, R. Nieuwland, Optical and non-optical methods for detection and characterization of microparticles and exosomes, J. Thromb. Haemost. 8 (2010) 2596-2607, https://doi.org/10.1111/j.1538-7836.2010.04074.x.

[221] R.A. Dragovic, C. Gardiner, A.S. Brooks, D.S. Tannetta, D.J. Ferguson, P. Hole, B. Carr, C.W. Redman, A.L. Harris, P.J. Dobson, P. Harrison, I.L. Sargent, Sizing and phenotyping of cellular vesicles using nanoparticle tracking analysis, Nanomedicine. 7 (2011) 780-788, https://doi.org/10.1016/ j.nano.2011.04.003.

[222] V. Filipe, A. Hawe, W. Jiskoot, Critical evaluation of Nanoparticle Tracking Analysis (NTA) by NanoSight for the measurement of nanoparticles and protein aggregates, Pharm. Res. 27 (2010) 796-810, https://doi.org/10.1007/ s11095-010-0073-2.

[223] A.S. Lawrie, A. Albanyan, R.A. Cardigan, I.J. Mackie, P. Harrison, Microparticle sizing by dynamic light scattering in fresh-frozen plasma, Vox Sang. 96 (2009) 206-212, https://doi.org/10.1111/j.1423-0410.2008.01151.x.

[224] E. van der Pol, F.A.W. Coumans, A.E. Grootemaat, C. Gardiner, I.L. Sargent, P. Harrison, A. Sturk, T.G. van Leeuwen, R. Nieuwland, Particle size distribution of exosomes and microvesicles determined by transmission electron microscopy, flow cytometry, nanoparticle tracking analysis, and resistive pulse sensing, J. Thromb. Haemost. 12 (2014) 1182-1192, https://doi.org/ $10.1111 /$ jth.12602.

[225] F.A.W. Coumans, A.R. Brisson, E.I. Buzas, F. Dignat-George, E.E.E. Drees, S. ElAndaloussi, C. Emanueli, A. Gasecka, A. Hendrix, A.F. Hill, R. Lacroix, Y. Lee, T. G. van Leeuwen, N. Mackman, I. Mäger, J.P. Nolan, E. van der Pol, D.M. Pegtel, S. Sahoo, P.R.M. Siljander, G. Sturk, O. de Wever, R. Nieuwland, Methodological guidelines to study extracellular vesicles, Circ. Res. 120 (2017) 1632-1648, https://doi.org/10.1161/CIRCRESAHA.117.309417.

[226] J. Webber, A. Clayton, How pure are your vesicles?, J Extracell. Vesicles. 2 (2013) 19861, https://doi.org/10.3402/jev.v2i0.19861.

[227] O.P. Wiklander, J.Z. Nordin, A. O’Loughlin, Y. Gustafsson, G. Corso, I. Mager, P. Vader, Y. Lee, H. Sork, Y. Seow, N. Heldring, L. Alvarez-Erviti, C.I. Smith, K. Le Blanc, P. Macchiarini, P. Jungebluth, M.J. Wood, S.E. Andaloussi, Extracellular vesicle in vivo biodistribution is determined by cell source, route of administration and targeting, J. Extracell. Vesicles. 4 (2015) 26316, https:// doi.org/10.3402/jev.v4.26316.

[228] J.W. Wang, Y.N. Zhang, S.K. Sze, S.M. van de Weg, F. Vernooij, A.H. Schoneveld, S.H. Tan, H.H. Versteeg, L. Timmers, C.S.P. Lam, D.P.V. de Kleijn, Lowering lowdensity lipoprotein particles in plasma using dextran sulphate coprecipitates procoagulant extracellular vesicles, Int. J. Mol. Sci. 19 (2017), https://doi.org/10.3390/ijms19010094.

[229] S. Senapati, A.K. Mahanta, S. Kumar, P. Maiti, Controlled drug delivery vehicles for cancer treatment and their performance, Signal Transduct. Target. Ther. 3 (2018) 1-19, https://doi.org/10.1038/s41392-017-0004-3.

[230] S. Svenson, Clinical translation of nanomedicines, Curr. Opin. Solid State Mater. Sci. 16 (2012) 287-294, https://doi.org/10.1016/j.cossms.2012.10.001.

[231] M. Cağdaş, A.D. Sezer, S. Bucak, Liposomes as Potential Drug Carrier Systems for Drug Delivery, InTech, 2014, http://doi.org/10.5772/58459.

[232] T.M. Allen, C.B. Hansen, D.E.L. de Menezes, Pharmacokinetics of longcirculating liposomes, Adv. Drug. Deliv. Rev. 16 (1995) 267-284, https:// doi.org/10.1016/0169-409X(95)00029-7.

[233] E. Gentile, F. Cilurzo, L. Di Marzio, M. Carafa, C. Anna Ventura, J. Wolfram, D. Paolino, C. Celia, Liposomal chemotherapeutics, Future Oncol. 9 (2013) 18491859, https://doi.org/10.2217/fon.13.146.

[234] S. Walker, S. Busatto, A. Pham, M. Tian, A. Suh, K. Carson, A. Quintero, M. Lafrence, H. Malik, M.X. Santana, J. Wolfram, Extracellular vesicle-based drug delivery systems for cancer treatment, Theranostics. 9 (2019) 8001-8017, https://doi.org/10.7150/thno.37097.

[235] M. Merino, S. Zalba, M.J. Garrido, Immunoliposomes in clinical oncology: state of the art and future perspectives, J. Control Release. 275 (2018) 162176, https://doi.org/10.1016/j.jconrel.2018.02.015.

[236] J.C. Kraft, J.P. Freeling, Z. Wang, R.J.Y. Ho, Emerging research and clinical development trends of liposome and lipid nanoparticle drug delivery systems, J. Pharm. Sci. 103 (2014) 29-52, https://doi.org/10.1002/jps.23773.

[237] E. Beltrán-Gracia, A. López-Camacho, I. Higuera-Ciapara, J.B. VelázquezFernández, A.A. Vallejo-Cardona, Nanomedicine review: clinical developments in liposomal applications, Cancer Nanotechnol. 10 (2019) 11, https://doi.org/10.1186/s12645-019-0055-y.

[238] T. Olusanya, R. Haj Ahmad, D. Ibegbu, J. Smith, A. Elkordy, Liposomal drug delivery systems and anticancer drugs, Molecules. 23 (2018) 907, https://doi. org/10.3390/molecules23040907.

[239] Y.H. Choi, H.-K. Han, Nanomedicines: current status and future perspectives in aspect of drug delivery and pharmacokinetics, J. Pharm. Investig. 48 (2018) 43-60, https://doi.org/10.1007/s40005-017-0370-4.

[240] S. Wilhelm, A.J. Tavares, Q. Dai, S. Ohta, J. Audet, H.F. Dvorak, W.C.W. Chan, Analysis of nanoparticle delivery to tumours, Nat. Rev. Mater. 1 (2016) 16014, https://doi.org/10.1038/natrevmats.2016.14.

[241] M. Longmire, P.L. Choyke, H. Kobayashi, Clearance properties of nano-sized particles and molecules as imaging agents: considerations and caveats,

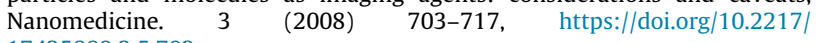
17435889.3.5.703.

[242] A. Nagayasu, K. Uchiyama, H. Kiwada, The size of liposomes: a factor which affects their targeting efficiency to tumors and therapeutic activity of liposomal antitumor drugs, Adv. Drug. Deliv. Rev. 40 (1999) 75-87, https:// doi.org/10.1016/S0169-409X(99)00041-1.
[243] G.J.R. Charrois, T.M. Allen, Rate of biodistribution of STEALTH ${ }^{\circledR}$ liposomes to tumor and skin: influence of liposome diameter and implications for toxicity and therapeutic activity, Biochim. Biophys. Acta. Biomembr. 1609 (2003) 102-108, https://doi.org/10.1016/S0005-2736(02)00661-2.

[244] J. Patel, Liposomal doxorubicin: Doxil ${ }^{\circledR}$, J. Oncol. Pharm. Pract. 2 (1996) 201 210, https://doi.org/10.1177/107815529600200402.

[245] J. Wang, D. Chen, E.A. Ho, Challenges in the development and establishment of exosome-based drug delivery systems, J. Control Release. (2020) 1-13, https://doi.org/10.1016/j.jconrel.2020.10.020.

[246] M.F. Attia, N. Anton, J. Wallyn, Z. Omran, T.F. Vandamme, An overview of active and passive targeting strategies to improve the nanocarriers efficiency to tumour sites, J. Pharm. Pharmacol. 71 (2019) 1185-1198, https://doi.org/ 10.1111/jphp.13098.

[247] S. Hua, S.Y. Wu, The use of lipid-based nanocarriers for targeted pain therapies, Front. Pharmacol. 4 (Nov) (2013) 1-7, https://doi.org/10.3389/ fphar.2013.00143.

[248] C. Rothkopf, A. Fahr, G. Fricker, G.L. Scherphof, J.A. Kamps, Uptake of phosphatidylserine-containing liposomes by liver sinusoidal endothelial cells in the serum-free perfused rat liver, Biochim Biophys Acta. 1668 (2005) 1016, https://doi.org/10.1016/j.bbamem.2004.10.013.

[249] J.A. Kamps, H.W. Morselt, P.J. Swart, D.K. Meijer, G.L. Scherphof, Massive targeting of liposomes, surface-modified with anionized albumins, to hepatic endothelial cells, Proc. Natl. Acad. Sci. U S A. 94 (1997) 11681-11685, https:// doi.org/10.1073/pnas.94.21.11681.

[250] T. Imai, Y. Takahashi, M. Nishikawa, K. Kato, M. Morishita, T. Yamashita, A. Matsumoto, C. Charoenviriyakul, Y. Takakura, Macrophage-dependent clearance of systemically administered B16BL6-derived exosomes from the blood circulation in mice, J. Extracell. Vesicles. 4 (2015) 26238, https://doi. org/10.3402/jev.v4.26238.

[251] T. Smyth, M. Kullberg, N. Malik, P. Smith-Jones, M.W. Graner, T.J. Anchordoquy, Biodistribution and delivery efficiency of unmodified tumorderived exosomes, J. Control Release. 199 (2015) 145-155, https://doi.org/ 10.1016/j.jconrel.2014.12.013.

[252] R. van der Meel, M.H. Fens, P. Vader, W.W. van Solinge, O. Eniola-Adefeso, R. M. Schiffelers, Extracellular vesicles as drug delivery systems: lessons from the liposome field, J. Control Release. 195 (2014) 72-85, https://doi.org/ 10.1016/j.jconrel.2014.07.049.

[253] P. Milla, F. Dosio, L. Cattel, PEGylation of proteins and liposomes: a powerful and flexible strategy to improve the drug delivery, Curr. Drug Metab. 13 (2012) 105-119, https://doi.org/10.2174/138920012798356934.

[254] S.A.A. Kooijmans, L.A.L. Fliervoet, R. van der Meel, M. Fens, H.F.G. Heijnen, P. M.P. van Bergen En Henegouwen, P. Vader, R.M. Schiffelers, PEGylated and targeted extracellular vesicles display enhanced cell specificity and circulation time, J. Control Release. 224 (2016) 77-85, https://doi.org/ 10.1016/j.jconrel.2016.01.009.

[255] D.E. Murphy, O.G. de Jong, M. Brouwer, M.J. Wood, G. Lavieu, R.M. Schiffelers, P. Vader, Extracellular vesicle-based therapeutics: natural versus engineered targeting and trafficking, Exp. Mol. Med. 51 (2019) 32, https://doi.org/ 10.1038/s12276-019-0223-5.

[256] M. Germain, M.E. Meyre, L. Poul, M. Paolini, C. Berjaud, F. Mpambani, M. Bergere, L. Levy, A. Pottier, Priming the body to receive the therapeutic agent to redefine treatment benefit/risk profile, Sci. Rep. 8 (2018) 1-11, https://doi. org/10.1038/s41598-018-23140-9.

[257] N.R.M. Saunders, M.S. Paolini, O.S. Fenton, L. Poul, J. Devalliere, F. Mpambani, A. Darmon, M. Bergère, O. Jibault, M. Germain, R. Langer, A nanoprimer to improve the systemic delivery of siRNA and mRNA, Nano Lett. 20 (2020) 4264-4269, https://doi.org/10.1021/acs.nanolett.0c00752.

[258] D.C. Watson, D. Bayik, A. Srivatsan, C. Bergamaschi, A. Valentin, G. Niu, J. Bear, M. Monninger, M. Sun, A. Morales-Kastresana, J.C. Jones, B.K. Felber, X. Chen, I. Gursel, G.N. Pavlakis, Efficient production and enhanced tumor delivery of engineered extracellular vesicles, Biomaterials. 105 (2016) 195-205, https:// doi.org/10.1016/j.biomaterials.2016.07.003.

[259] E.T.M. Dams, P. Laverman, W.J.G. Oyen, G. Storm, G.L. Scherphof, J.W.M. Van Der Meer, F.H.M. Corstens, O.C. Boerman, Accelerated blood clearance and altered biodistribution of repeated injections of sterically stabilized liposomes, J. Pharmacol. Exp. Ther. 292 (2000) 1071-1079.

[260] A.S. Abu Lila, H. Kiwada, T. Ishida, The accelerated blood clearance (ABC) phenomenon: clinical challenge and approaches to manage, J. Control Release. 172 (2013) 38-47, https://doi.org/10.1016/j.jconrel.2013.07.026.

[261] Y. Mima, Y. Hashimoto, T. Shimizu, H. Kiwada, T. Ishida, Anti-PEG IgM is a major contributor to the accelerated blood clearance of polyethylene glycolconjugated protein, Mol. Pharm. 12 (2015) 2429-2435, https://doi.org/ 10.1021/acs.molpharmaceut.5b00144.

[262] A.E. Hansen, A.L. Petersen, J.R. Henriksen, B. Boerresen, P. Rasmussen, D.R Elema, P.M.a. Rosenschöld, A.T. Kristensen, A. Kjær, T.L. Andresen, Positron emission tomography based elucidation of the enhanced permeability and retention effect in dogs with cancer using Copper-64 liposomes, ACS Nano. 9 (2015) 6985-6995, https://doi.org/10.1021/acsnano.5b01324.

[263] H. Koide, T. Asai, K. Hatanaka, T. Urakami, T. Ishii, E. Kenjo, M. Nishihara, M. Yokoyama, T. Ishida, H. Kiwada, N. Oku, Particle size-dependent triggering of accelerated blood clearance phenomenon, Int. J. Pharm. 362 (2008) 197-200, https://doi.org/10.1016/j.ijpharm.2008.06.004.

[264] T. Ishihara, M. Takeda, H. Sakamoto, A. Kimoto, C. Kobayashi, N. Takasaki, K. Yuki, K.I. Tanaka, M. Takenaga, R. Igarashi, T. Maeda, N. Yamakawa, Y. Okamoto, M. Otsuka, T. Ishida, H. Kiwada, Y. Mizushima, T. Mizushima, 
Accelerated blood clearance phenomenon upon repeated injection of pegmodified pla-nanoparticles, Pharm. Res. 26 (2009) 2270-2279, https://doi. org/10.1007/s11095-009-9943-X.

[265] T. Ishihara, T. Maeda, H. Sakamoto, N. Takasaki, M. Shigyo, T. Ishida, H. Kiwada, Y. Mizushima, T. Mizushima, Evasion of the accelerated blood clearance phenomenon by coating of nanoparticles with various hydrophilic polymers, Biomacromolecules. 11 (2010) 2700-2706, https://doi.org/ 10.1021/bm100754e.

[266] Z. Zhang, Y. Chu, C. Li, W. Tang, J. Qian, X. Wei, W. Lu, T. Ying, C. Zhan, AntiPEG ScFv corona ameliorates accelerated blood clearance phenomenon of PEGylated nanomedicines, J. Control Release. 330 (2021) 493-501, https:// doi.org/10.1016/j.jconrel.2020.12.047.

[267] E. Örfi, T. Mészáros, M. Hennies, T. Fülöp, L. Dézsi, A. Nardocci, L. Rosivall, P. Hamar, B.W. Neun, M.A. Dobrovolskaia, J. Szebeni, G. Szénási, Acute physiological changes caused by complement activators and amphotericin b-containing liposomes in mice, Int. J. Nanomedicine. 14 (2019) 1563-1573, https://doi.org/10.2147/IJN.S187139.

[268] J. Szebeni, Complement activation-related pseudoallergy: A new class of drug-induced acute immune toxicity, Toxicology. 216 (2005) 106-121, https://doi.org/10.1016/j.tox.2005.07.023.

[269] L.R. Sharma, A. Subedi, B.K. Shah, Anaphylaxis to pegylated liposomal doxorubicin: a case report, West Indian Med. J. 63 (2014) 376-377, https:// doi.org/10.7727/wimj.2013.270.

[270] G. Milosevits, J. Szebeni, S. Krol, Exosomes: potential model for complementstealth delivery systems, Eur. J. Nanomed. 7 (2015), https://doi.org/10.1515/ ejnm-2015-0005.

[271] G. Ferrandina, M. Ludovisi, D. Lorusso, S. Pignata, E. Breda, A. Savarese, P. Del Medico, L. Scaltriti, D. Katsaros, D. Priolo, G. Scambia, Phase III trial of gemcitabine compared with pegylated liposomal doxorubicin in progressive or recurrent ovarian cancer, J. Clin. Oncol. 26 (2008) 890-896, https://doi.org/ 10.1200/JCO.2007.13.6606.

[272] M.A. Morse, J. Garst, T. Osada, S. Khan, A. Hobeika, T.M. Clay, N. Valente, R. Shreeniwas, M.A. Sutton, A. Delcayre, D.-H.H. Hsu, J.-B.B. Le Pecq, H.K. Lyerly, A phase I study of dexosome immunotherapy in patients with advanced nonsmall cell lung cancer, J. Transl. Med. 3 (2005) 9, https://doi.org/10.1186/ 1479-5876-3-9.

[273] B. Escudier, T. Dorval, N. Chaput, F. André, M.P. Caby, S. Novault, C. Flament, C. Leboulaire, C. Borg, S. Amigorena, C. Boccaccio, C. Bonnerot, O. Dhellin, M. Movassagh, S. Piperno, C. Robert, V. Serra, N. Valente, J.B. Le Pecq, A. Spatz, O. Lantz, T. Tursz, E. Angevin, L. Zitvogel, Vaccination of metastatic melanoma patients with autologous dendritic cell (DC) derived-exosomes: results of the first phase 1 clinical trial, J. Transl. Med. 3 (2005) 1-13, https://doi.org/ 10.1186/1479-5876-3-10.

[274] K.B. Johnsen, J.M. Gudbergsson, M. Duroux, T. Moos, T.L. Andresen, J.B. Simonsen, On the use of liposome controls in studies investigating the clinical potential of extracellular vesicle-based drug delivery systems - A commentary, J. Control Release. 269 (2018) 10-14, https://doi.org/10.1016/j. jconrel.2017.11.002.

[275] V.P. Torchilin, Recent advances with liposomes as pharmaceutical carriers, Nat. Rev. Drug Discov. 4 (2005) 145-160, https://doi.org/10.1038/nrd1632.

[276] I.E. Allijn, B.M.S. Czarny, X. Wang, S.Y. Chong, M. Weiler, A.E. da Silva, J.M. Metselaar, C.S.P. Lam, G. Pastorin, D.P.V. de Kleijn, G. Storm, J.W. Wang, R.M. Schiffelers, Liposome encapsulated berberine treatment attenuates cardiac dysfunction after myocardial infarction, J. Control Release. 247 (2017) 127133, https://doi.org/10.1016/j.jconrel.2016.12.042.

[277] S. Sindhwani, A.M. Syed, J. Ngai, B.R. Kingston, L. Maiorino, J. Rothschild, P. MacMillan, Y. Zhang, N.U. Rajesh, T. Hoang, J.L.Y. Wu, S. Wilhelm, A. Zilman, S. Gadde, A. Sulaiman, B. Ouyang, Z. Lin, L. Wang, M. Egeblad, W.C.W. Chan, The entry of nanoparticles into solid tumours, Nat. Mater. 19 (2020) 566-575, https://doi.org/10.1038/s41563-019-0566-2.

[278] B.M. Bell, I.D. Kirk, S. Hiltbrunner, S. Gabrielsson, J.J. Bultema, Designer exosomes as next-generation cancer immunotherapy, Nanomedicine. 12 (2016) 163-169, https://doi.org/10.1016/j.nano.2015.09.011.

[279] Y. Yang, X. Tai, K. Shi, S. Ruan, Y. Qiu, Z. Zhang, B. Xiang, Q. He, A new concept of enhancing immuno-chemotherapeutic effects against B16F10 tumor via systemic administration by taking advantages of the limitation of EPR effect, Theranostics. 6 (2016) 2141-2160, https://doi.org/10.7150/ thno.16184.

[280] J. Wolfram, M. Ferrari, Clinical cancer nanomedicine, Nano Today. 25 (2019) 85-98, https://doi.org/10.1016/j.nantod.2019.02.005.

[281] D.R. Beckford Vera, S.D. Fontaine, H.F. VanBrocklin, B.R. Hearn, R. Reid, G.W. Ashley, D.V. Santi, PET imaging of the EPR effect in tumor xenografts using small $15 \mathrm{~nm}$ diameter polyethylene glycols labeled with zirconium-89, Mol. Cancer Ther. 19 (2020) 673-679, https://doi.org/10.1158/1535-7163.MCT19-0709.

[282] A. Hoshino, B. Costa-Silva, T.-L. Shen, G. Rodrigues, A. Hashimoto, M. Tesic Mark, H. Molina, S. Kohsaka, A. Di Giannatale, S. Ceder, S. Singh, C. Williams, N. Soplop, K. Uryu, L. Pharmer, T. King, L. Bojmar, A.E. Davies, Y. Ararso, T. Zhang, H. Zhang, J. Hernandez, J.M. Weiss, V.D. Dumont-Cole, K. Kramer, L.H. Wexler, A. Narendran, G.K. Schwartz, J.H. Healey, P. Sandstrom, K. Jørgen Labori, E.H. Kure, P.M. Grandgenett, M.A. Hollingsworth, M. de Sousa, S. Kaur, M. Jain, K. Mallya, S.K. Batra, W.R. Jarnagin, M.S. Brady, O. Fodstad, V. Muller, K. Pantel, A.J. Minn, M.J. Bissell, B.A. Garcia, Y. Kang, V.K. Rajasekhar, C.M. Ghajar, I. Matei, H. Peinado, J. Bromberg, D. Lyden, Tumour exosome integrins determine organotropic metastasis, Nature. 527 (2015) 329-335, https://doi. org/10.1038/nature15756.
[283] M. Tsuchihashi, H. Harashima, H. Kiwada, Development of a pharmacokinetic/pharmacodynamic (PK/PD)-simulation system for doxorubicin in long circulating liposomes in mice using peritoneal P388, J. Control Release. 61 (1999) 9-19, https://doi.org/10.1016/S0168-3659(99) 00103-0.

[284] A. Gabizon, R. Catane, B. Uziely, B. Kaufman, T. Safra, R. Cohen, F. Martin, A. Huang, Y. Barenholz, Prolonged circulation time and enhanced accumulation in malignant exudates of doxorubicin encapsulated in polyethylene-glycol coated liposomes, Cancer Res, 54 (1994) 987-992.

[285] F. Martin, A. Huang, B. Uziely, B. Kaufman, T. Safra, Prolonged circulation time and enhanced accumulation in malignant exudates of doxorubicin encapsulated in polyethylene-glycol coated liposomes, Cancer Res. 54 (1994) 987-992.

[286] J. Zhang, F. Leifer, S. Rose, D.Y. Chun, J. Thaisz, T. Herr, M. Nashed, J. Joseph, W. R. Perkins, K. DiPetrillo, Amikacin liposome inhalation suspension (ALIS) penetrates non-tuberculous mycobacterial biofilms and enhances amikacin uptake into macrophages, Front. Microbiol. 9 (2018) 1-12, https://doi.org/ 10.3389/fmicb.2018.00915.

[287] C. Charoenviriyakul, Y. Takahashi, M. Morishita, A. Matsumoto, M. Nishikawa, Y. Takakura, Cell type-specific and common characteristics of exosomes derived from mouse cell lines: yield, physicochemical properties, and pharmacokinetics, Eur. J. Pharm. Sci. 96 (2017) 316-322, https://doi.org/ 10.1016/j.ejps.2016.10.009.

[288] K.W. Witwer, Extracellular vesicles versus synthetic nanoparticles for drug delivery, Nat. Rev. Mater., http://doi.org/10.1038/s41578-020-00277-6.

[289] R. Munter, K. Kristensen, D. Pedersbaek, J.B. Larsen, J.B. Simonsen, T.L Andresen, Dissociation of fluorescently labeled lipids from liposomes in biological environments challenges the interpretation of uptake studies, Nanoscale. 10 (2018) 22720-22724, https://doi.org/10.1039/c8nr07755j.

[290] P. Gangadaran, C.M. Hong, B.-C. Ahn, Current perspectives on in vivo noninvasive tracking of extracellular vesicles with molecular imaging, Biomed. Res. Int. 2017 (2017) 1-11, https://doi.org/10.1155/2017/9158319.

[291] H. Choi, D.S. Lee, Illuminating the physiology of extracellular vesicles, Stem Cell Res. Ther. 7 (2016) 55, https://doi.org/10.1186/s13287-016-0316-1.

[292] W. Luo, Y. Dai, Z. Chen, X. Yue, K.C. Andrade-Powell, J. Chang, Spatial and temporal tracking of cardiac exosomes in mouse using a nano-luciferaseCD63 fusion protein, Commun. Biol. 3 (2020) 114, https://doi.org/10.1038/ s42003-020-0830-7.

[293] A.B. Keener, How extracellular vesicles can enhance drug delivery, Nature. 582 (2020) S14-S15, https://doi.org/10.1038/d41586-020-01769-9.

[294] S.A.A. Kooijmans, S. Stremersch, K. Braeckmans, S.C. de Smedt, A. Hendrix, M. J.A. Wood, R.M. Schiffelers, K. Raemdonck, P. Vader, Electroporation-induced SiRNA precipitation obscures the efficiency of siRNA loading into extracellular vesicles, J. Control Release. 172 (2013) 229-238, https://doi. org/10.1016/j.jconrel.2013.08.014.

[295] D.S. Sutaria, J. Jiang, O.A. Elgamal, S.M. Pomeroy, M. Badawi, X. Zhu, R. Pavlovicz, A.C.P. Azevedo-Pouly, J. Chalmers, C. Li, M.A. Phelps, T.D. Schmittgen, Low active loading of cargo into engineered extracellular vesicles results in inefficient miRNA mimic delivery, J. Extracell. Vesicles. 6 (2017) 1333882, https://doi.org/10.1080/20013078.2017.1333882.

[296] S. Bosch, L. de Beaurepaire, M. Allard, M. Mosser, C. Heichette, D. Chretien, D. Jegou, J.M. Bach, Trehalose prevents aggregation of exosomes and cryodamage, Sci. Rep. 6 (2016) 36162, https://doi.org/10.1038/srep36162.

[297] D.E. Murphy, O.G. de Jong, M.J.W. Evers, M. Nurazizah, R.M. Schiffelers, P. Vader, Natural or synthetic RNA delivery: a stoichiometric comparison of extracellular vesicles and synthetic nanoparticles, Nano Lett. (2021), https:// doi.org/10.1021/acs.nanolett.1c00094, acs.nanolett.1c00094acs.nanolett.00091c00094.

[298] A. Vandergriff, K. Huang, D. Shen, S. Hu, M.T. Hensley, T.G. Caranasos, L. Qian, $\mathrm{K}$. Cheng, Targeting regenerative exosomes to myocardial infarction using cardiac homing peptide, Theranostics. 8 (2018) 1869-1878, https://doi.org/ $10.7150 /$ thno.20524.

[299] X.T.T. Dang, J.M. Kavishka, D.X. Zhang, M. Pirisinu, M.T.N. Le, Extracellular vesicles as an efficient and versatile system for drug delivery, Cells. 9 (2020) 2191, https://doi.org/10.3390/cells9102191.

[300] O.G. de Jong, D.E. Murphy, I. Mäger, E. Willms, A. Garcia-Guerra, J.J. GitzFrancois, J. Lefferts, D. Gupta, S.C. Steenbeek, J. van Rheenen, S. El Andaloussi, R.M. Schiffelers, M.J.A. Wood, P. Vader, A CRISPR-Cas9-based reporter system for single-cell detection of extracellular vesicle-mediated functional transfer of RNA, Nat. Commun. 11 (2020) 1113, https://doi.org/10.1038/s41467-02014977-8.

[301] W.J. Goh, S. Zou, C.K. Lee, Y.-H. Ou, J.-W. Wang, B. Czarny, G. Pastorin, EXOPLEXs: chimeric drug delivery platform from the fusion of cell-derived nanovesicles and liposomes, Biomacromolecules. 19 (2018) 22-30, https:// doi.org/10.1021/acs.biomac.7b01176.

[302] Y.-H. Ou, S. Zou, W.J. Goh, S.Y. Chong, G. Venkatesan, M.G. Wacker, G. Storm, J.-W. Wang, B. Czarny, G. Pastorin, E.C.Y. Woon, Micro cell vesicle technology (mCVT): a novel hybrid system of gene delivery for hard-to-transfect (HTT) cells, Nanoscale. 12 (2020) 18022-18030, https://doi.org/10.1039/ DONR03784B.

[303] J. Gilleron, W. Querbes, A. Zeigerer, A. Borodovsky, G. Marsico, U. Schubert, K. Manygoats, S. Seifert, C. Andree, M. Stöter, H. Epstein-Barash, L. Zhang, V. Koteliansky, K. Fitzgerald, E. Fava, M. Bickle, Y. Kalaidzidis, A. Akinc, M. Maier, M. Zerial, Image-based analysis of lipid nanoparticle-mediated siRNA delivery, intracellular trafficking and endosomal escape, Nat. Biotechnol. 31 (2013) 638-646, https://doi.org/10.1038/nbt.2612. 
[304] B.S. Joshi, M.A. de Beer, B.N.G. Giepmans, I.S. Zuhorn, Endocytosis of extracellular vesicles and release of their cargo from endosomes, ACS Nano. 14 (2020) 4444-4455, https://doi.org/10.1021/acsnano.9b10033.

[305] E. Bonsergent, G. Lavieu, Content release of extracellular vesicles in a cell-free extract, FEBS. Lett. 593 (2019) 1983-1992, https://doi.org/10.1002/18733468.13472.

[306] R.L. Webb, E.E. Kaiser, S.L. Scoville, T.A. Thompson, S. Fatima, C. Pandya, K. Sriram, R.L. Swetenburg, K. Vaibhav, A.S. Arbab, B. Baban, K.M. Dhandapani, D. C. Hess, M.N. Hoda, S.L. Stice, Human neural stem cell extracellular vesicles improve tissue and functional recovery in the murine thromboembolic stroke model, Transl. Stroke Res. 9 (2018) 530-539, https://doi.org/10.1007/s12975017-0599-2.

[307] Z. Han, S. Liu, Y. Pei, Z. Ding, Y. Li, X. Wang, D. Zhan, S. Xia, T. Driedonks, K.W. Witwer, R.G. Weiss, P.C.M. Zijl, J.W.M. Bulte, L. Cheng, G. Liu, Highly efficient magnetic labelling allows MRI tracking of the homing of stem cell-derived extracellular vesicles following systemic delivery, J. Extracell. Vesicles. 10 (2021), https://doi.org/10.1002/jev2.12054.

[308] L. Peruzzotti-Jametti, J.D. Bernstock, G. Manferrari, R. Rogall, E. FernandezVizarra, J.C. Williamson, A. Braga, A. van den Bosch, T. Leonardi, Á. Kittel, C. Benincá, N. Vicario, S. Tan, C. Bastos, I. Bicci, N. Iraci, J.A. Smith, P.J. Lehner, E.I. Buzas, N. Faria, M. Zeviani, C. Frezza, A. Brisson, N.J. Matheson, C. Viscomi, S. Pluchino, Neural stem cells traffic functional mitochondria via extracellular vesicles to correct mitochondrial dysfunction in target cells, BioRxiv. (2020), https://doi.org/10.1101/2020.01.29.923441.

[309] J. Silva, L.M.A. Murray, Y. Su, D.F. Mc Auley, C.M. O’Kane, A. Krasnodembskaya, Transfer of mitochondria from mesenchymal stromal cells through extracellular vesicles improves alveolar epithelial-capillary barrier in ARDS, Eur. Respir. Soc., pp. 98-98, http://doi.org/10.1183/23120541.LSC-2020.98.
[310] G. Ikeda, M.R. Santoso, Y. Tada, A.M. Li, E. Vaskova, J.-H. Jung, C. O’Brien, E. Egan, J. Ye, P.C. Yang, Mitochondria-rich extracellular vesicles from autologous stem cell-derived cardiomyocytes restore energetics of ischemic myocardium, J. Am. Coll. Cardiol. 77 (2021) 1073-1088, https://doi.org/ 10.1016/j.jacc.2020.12.060.

[311] P. Wolf, The nature and significance of platelet products in human plasma, $\mathrm{Br}$. J. Haematol. 13 (1967) 269-288, https://doi.org/10.1111/j.1365-2141.1967. tb08741.x.

[312] S. Aaronson, U. Behrens, R. Orner, T.H. Haines, Ultrastructure of intracellular and extracellular vesicles, membranes, and myelin figures produced by Ochromonas danica, J. Ultrastruct Res. 35 (1971) 418-430, https://doi.org/ 10.1016/s0022-5320(71)80003-5.

[313] E.G. Trams, C.J. Lauter, N. Salem Jr., U. Heine, Exfoliation of membrane ectoenzymes in the form of micro-vesicles, Biochim Biophys Acta. 645 (1981) 6370, https://doi.org/10.1016/0005-2736(81)90512-5.

[314] B.T. Pan, R.M. Johnstone, Fate of the transferrin receptor during maturation of sheep reticulocytes in vitro: selective externalization of the receptor, Cell. 33 (1983) 967-978, https://doi.org/10.1016/0092-8674(83)90040-5.

[315] C. Harding, J. Heuser, P. Stahl, Receptor-mediated endocytosis of transferrin and recycling of the transferrin receptor in rat reticulocytes, J. Cell Biol. 97 (1983) 329-339, https://doi.org/10.1083/jcb.97.2.329.

[316] L. Zitvogel, A. Regnault, A. Lozier, J. Wolfers, C. Flament, D. Tenza, P. RicciardiCastagnoli, G. Raposo, S. Amigorena, Eradication of established murine tumors using a novel cell-free vaccine: dendritic cell-derived exosomes, Nat. Med. 4 (1998) 594-600, https://doi.org/10.1038/nm0598-594.

[317] T. Pisitkun, R.F. Shen, M.A. Knepper, Identification and proteomic profiling of exosomes in human urine, Proc. Natl. Acad. Sci. U S A. 101 (2004) 13368 13373, https://doi.org/10.1073/pnas.0403453101. 